SAND88-2466

TTC 0836

DE91 002563

UNLIMITED REIEASE

UC-606

\title{
A BALLOON-BORNE AEROSOL SPECTROMETER FOR HIGH ALTITUDE \\ LOW AEROSOL CONCENTRATION MEASUREMENTS
}

G. S. Brown

Sandia National Laboratories

Albuquerque, NM

R. E. Weiss

Radiance Research

Seattle, WA

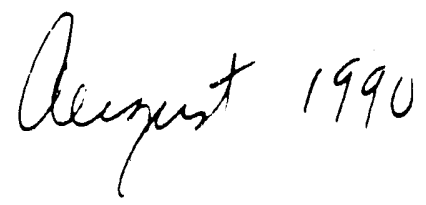

\footnotetext{
ABSTRACT

Funded by Air Force Wright Aeronautical Laboratory, a new balloon-borne high altitude aerosol spectrometer, for the measurement of cirrus cloud ice crystals, has been developed and sucessfully flown by Sandia National. Labcratories and Radiance Research. This report (1) details the aerosol spectrometer design and construction, (2) discusses data transmission and decoding, (3) presents data collected on three florida flights in tables and plots. 


\section{ACKNOWLEGEMENTS}

working within a limited budget and under severe time constraints many individuals contributed to the sucess of this project. Captain Janet Sorlin-Davis of the Air Force was a key individual who by her enthusiasm and support for a new technology insured a sucessful conclusion to a difficult project. The flight and data retrieval phase of the project was professionally accomplished by the personnel of the U. $S$. Air Force 6 th Weather squadron. The skilled technical support provided by Kim Rowland of Spectra Research allowed for timely completion of project milestones. Field trial logistics were expertly handled by Chuck Keady. A special thanks to Bernie zak, Applied Atmospheric Rewearch Program Coordinator, and Robert Luna, Risk Assesment and Transportation Systems Analysis Division Supervisor, for their interest, support and encouragement throughout the project. 


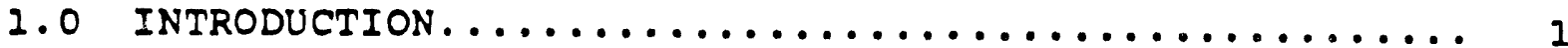

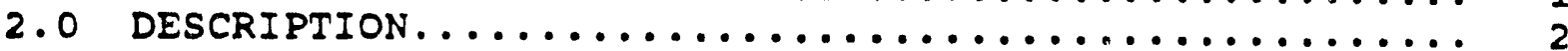

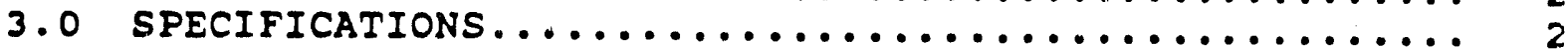

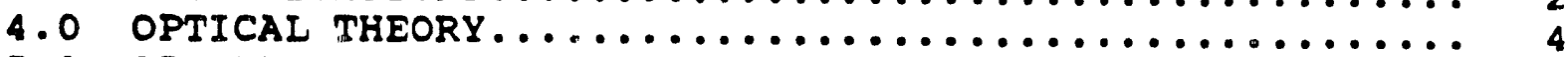

5.0 SIGNAI PROCESSING........................ 7

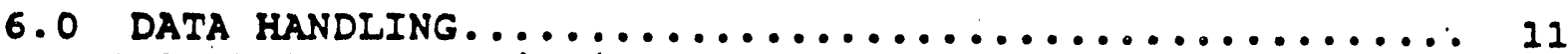

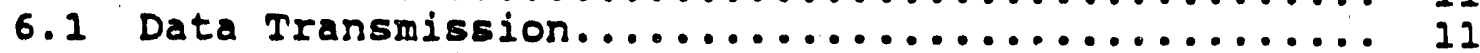

6.2 Data Processing......................... II

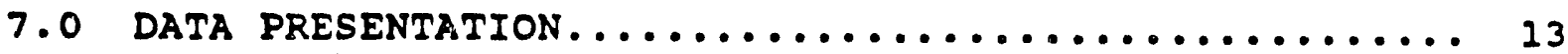

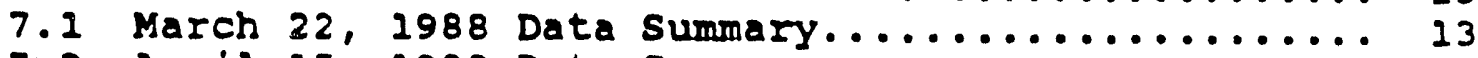

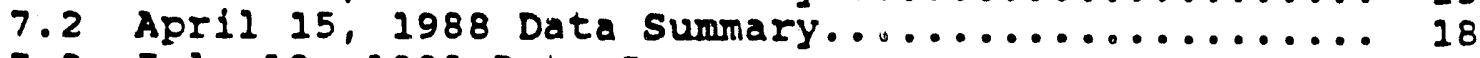

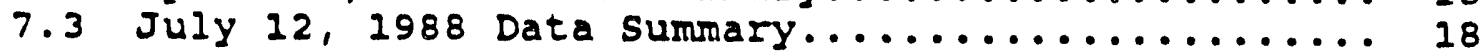

8.0 CONCLUSION............................ 28

APPENDIX A - Florida Data....................... 30

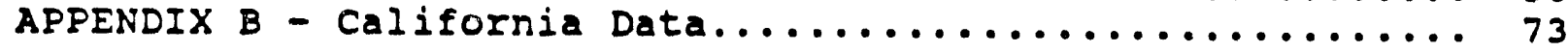

\section{FIGURES}

1. Aerosol spectrometer........................ 3

2. Aerosol spectrometer Block Diagram.................. 10

3. Vertical Temperature Profile March 22, 1988......... 15

4. Particle Concentration Distribution March 22, 1988... 16

5. Particle Size Distribution March 22, 1988.......... 17

6. Particle Concentration Distribution April 15, 1988... 19

7. Vertical Temperature Profile April 15, $1988 \ldots . . \ldots \ldots .20$

8. Particle Size Distribution April $15, \$ 988 \ldots \ldots \ldots \ldots . . . .21$

9. Vertical Temperature Profile July $12,1988 \ldots \ldots \ldots \ldots \ldots 22$

10. Particle Concentration Distribution July $12,1988 \ldots \ldots 23$

11. Particle Size Distribution July $12,1988 \ldots \ldots \ldots \ldots . \ldots 24$

\section{TABLES}

1. Concentration Distribution March $22,1988 \ldots \ldots \ldots \ldots 25$

2. Particle Size Relative Frequency March 22, 1988..... 26

3. Particle Size Relative Frequency July 12, 1988...... 27 


\subsection{INTRODOCTION}

Air Force wriaht Aeronautical Laboratory (AFWAL) tests of new IR sensor system (Infrared search and Track set, IRSTS) indicated significant discrepancies between IR transmission calculations obtained from the LOWTRAN code and observations made with the IRSTS system under test. IR transmission values obtained from the LOWTRAN code depended heavily upon atmospheric default parameters (atmospheric models) bilit into the code which made calculations possible in the absence of actual measurements. The calculations were made using default values, because no appropriate instrumentation for measuring the relevant parameters as a function of altitude were available. Thus these tests were conducted without adequate atmospheric characterization. Two of the parameters that were suspected causes of IR transmission loss were water vapor and high altitude thin cirrus.

The need for accurate atmospheric cirrus measurements provicied funding for joint development by sandia and Radiance Research of a balloon-borne high altitude low concentration aerosol spectrometer. The scope of this report includes aerosol spectrometer design and optical theory, data transmission/conversion format, and data presentation.

A balloon-borne frost-point hygrometer was also developed under this contract to provide atmospheric water vapor measurements and is detailed in SAND88-2467. 


\subsection{DESCRIPTION}

The aerosol spectrometer is designed to size and count large particles from ground level up to 35 kilometers altitude. The instrument can roughly size particles from a few micrometers effective diameter to over 50 micrometers effective diameter at concentrations less than $100 \mathrm{~m}^{-3}$.

The principal components of the spectrometer are a scattering chamber, light source, collimatig optics, dark trap, photo-multiplier tube, signal processing circuitry and power supplies (Figure 1). An external telemetry module is suspended below the aerosol sensor.

\subsection{BPECIFICATIONS}

\begin{tabular}{|c|c|}
\hline $\begin{array}{l}\text { Performance: } \\
\text { Minimum concentration: }\end{array}$ & $100 m^{-3}$ \\
\hline Maximum concentration: & $500,000 \mathrm{~m}^{-3}$ \\
\hline Outputs: & $\begin{array}{l}\text { 0-5 vDC, } 16 \text { bit resolution } \\
\text { 1. Flash count } \\
\text { 2. Size bin } 1 \\
\text { 3. Size bin } 2 \text { 13 } 13-26 \mathrm{um} \\
\text { 4. Size bin } 3 \text { 26-50 um } \\
\text { 5. Size bin } 4 \text { >50 um } \\
\text { 6. Total light scatter } \\
\text { 7. Reference light level }\end{array}$ \\
\hline Gross weight: & 1750 grams \\
\hline Power: & 2 watts ( $200 \mathrm{ma} 10 \mathrm{VDC})$ \\
\hline Dimensions: & $60 \mathrm{cra} \times 13 \mathrm{~cm} \times 17 \mathrm{~cm}$ \\
\hline
\end{tabular}


Stratospheric Particle Spectrometer

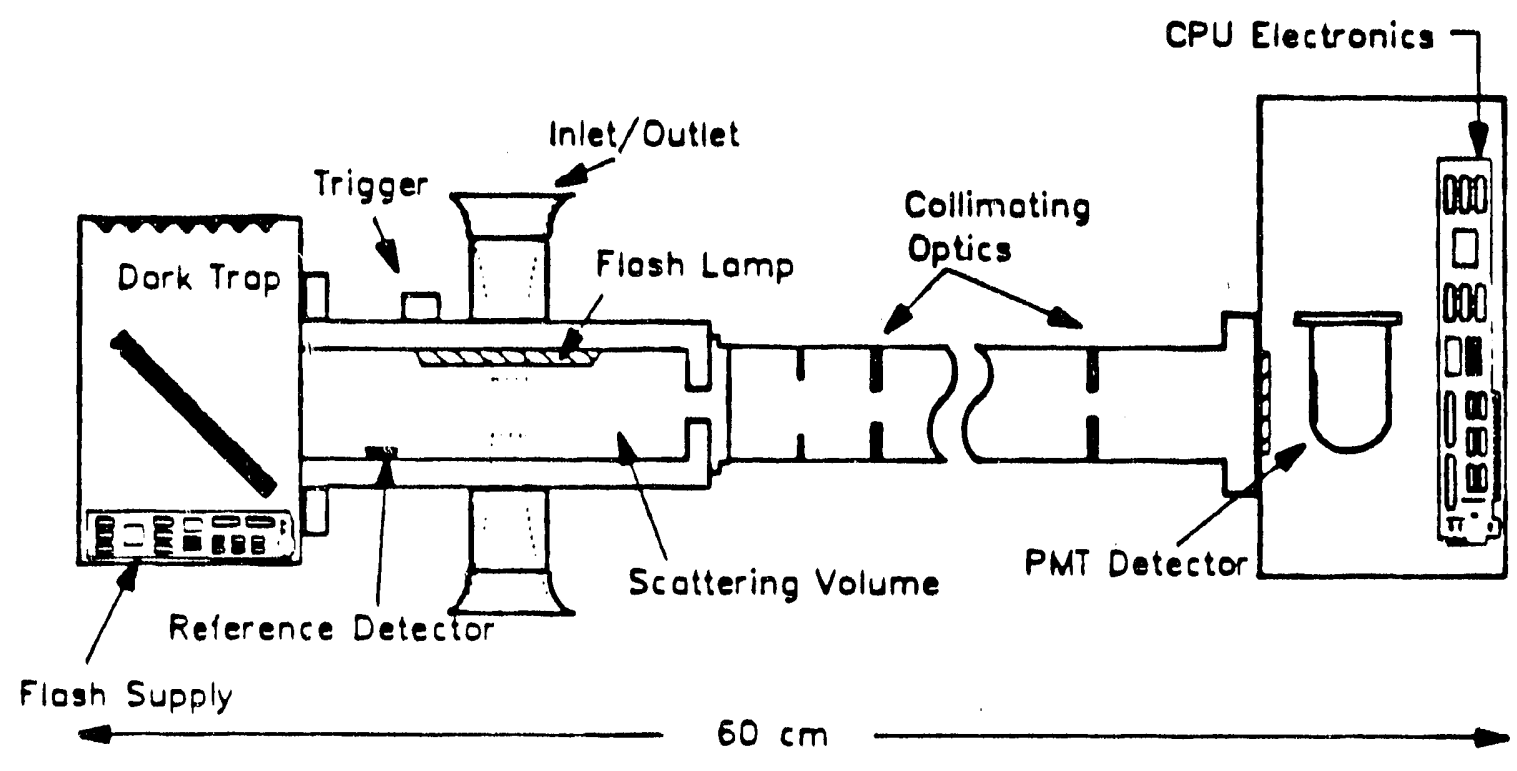

Figure 1. Aerosol spectrameter. 
The high altitude aerosol spectrometer is a single particle optical counter that detects the light scattered from individual part!cles larger than a few micrometers and relates the magnitude of that light to particle size. Particles that can be detected optically range in ize from 0.1 um to over $100 \mathrm{um}$. No single instrument is capable of measuring all sizes and concentrations. Each optical design must be adapted to work over a limited size and concentration range. As with other single particle optical counters, the configuration of the scattering chamber and illumination geometry in the aerosol spectrometer determine the performance limits of the instrument. Both particle size and concentration are determined to some extent by the size of the sensing volume. If the volume is too small, the count rate is much less than optimum for observed particle concentrations, and if too large, coincidence counting becomes a problem. Since the high altitude aerosol spectrometer is required to detect particle concentrations down to $100 \mathrm{~m}^{-3}$ and operate at low power levels, precluding the use of a continuous high intensity source, a novel optical design is required.

The optical design of the high altitude aerosol spectrometer is based on uniformly illuminating a large volume with a pulsed high intensity light source and then detecting a portion of the scattered light over a large scattering angle. The light source used is broad spectrum with wavelengths small compared to the particles to be detected. For large particles, the total optical cross section is simply proportional to the particle geometric cross section. The scattering volume is approximately $2 \mathrm{~cm}^{3}$ and $1 \mathrm{~s}$ illuminated by a 0.1 joule/flash xenon flash tube operating at $20 \mathrm{~Hz}$. The enclosed area surrounding the sensing volume is a very white, diffusely reflecting surface that provides relatively uniform illumination of the chamber. At a $20 \mathrm{~Hz}$ flash rate, 
2.4 liters are sampled each minute with an average power consumption of about 2 watts. Three lithium $c$ cells operating nominally at 10 VDC provide adequate power for a minimum of 10 hours flight time.

Because of the large size of the scattering volume, the particle position in the chamber may affect the magnitude of the detected light signal. This effect dictates particle size resolution and the number of size channels that can be discriminated. The scattering volume is about $3 \mathrm{~cm}$ long, on axis with the phototube, therefore light scattered by particles farther from the phototube will be reduced due to inverse square loss. For this reason, the detector is located as far from the scattering volume as feasible (35 cm). Maximum variation in light intensity at the detector due to axial position of the particle is about 15\%. However, the scattering cross section for large particles is proportional to the square of the particle radius. Therefore the actual error in size detection due to axial position of the particle is less than $10 \%$.

During each flash, two detectors are used to determine the relative scattered light intensity. A high gain photomultiplier tube (PMT) is used to detect the scattered photons against a dark background (dark trap). A GaAs reference photodetector is located in the scattering chamber to mersure the illumination intensity. The reference detector is used to normalize each flash to a constant intensity. Flash tubes exhibit a flash to flash variation in brightness. The reference detector is needed to remove the effect of that variation.

The actual light detected includes Rayleigh sattering, scattering by fine aerosol, scattering by coarse aerosol and wall scatter. To determine scattering by coarse aerosol only, all sources of background must be subtracted from the signal. 
The detectable linits in concentration are set by the size of the sensing chamber and the flash rate. The maximum concentration that can be detected without coincidence counting is $0.5 \mathrm{~cm}^{-3}$, dictated by the sample volume of $2 \mathrm{~cm}^{3}$. However, the practical limitation is about $0.2 \mathrm{~cm}^{-3}$ because the background suppression algorithm requires that large particles not be in the sensing volume for the majority of flashes. The minimum concentration is determined by the effective sampling rate:

$$
\begin{aligned}
& \text { Sample Rate }=\text { Flash Rate } \times \text { Sensing Volume } \\
& \text { Sample Rate }=20 \mathrm{~Hz} \times 2 \mathrm{~cm}^{3}=2.4 \text { liter } / \mathrm{min}
\end{aligned}
$$

During a five minute ascent sampling period the aerosol sensor can detect a particle concentration of slightly less than 100 $m^{-3}$, assuming a sample exchange rate of near $2.4 \mathrm{litre} / \mathrm{min}$. If a much greater exchange rate is used, there is the possibility of a particle not being counted. If a lesser exchange rate prevaiss, coincidence counting may occur. 


\subsection{BIGNAL PROCEBBING}

Signal processing is based on a Motorola $63 \mathrm{~A} 03$ single chip microprocessor. Code is written in 6800 assembler language and stored in a $8 \mathrm{kx}$ UV EPROM. The microprocessor controls all essential aspects of system operation including detector pulse integration, channel discrimination, background and dark current suppression, data output and flash rate control. The electronics are contalned on two circuit boards. The main circuit board contains the processor, photomultiplier tube (PMT), high voltage supply and the input/output (I/O) circuitry. The main circuit board is located in the PMT enclosure found at the detector end of the device. The second board contains the flash lamp supply and triggering circuitry and is located in the dark trap enclosure, away from the PMT and processor to reduce noise. All high voltage connections, incluaing the flash tube are potted for low pressure operation.

At power up, the flash tube begins to flash at $20 \mathrm{~Hz}$, as preset in software. If the input voltage is low, the unit will continue to operate, however, at a lower flash rate. Prior to each flash, the voltage on the flash tube storage capacitor is tested. If adequate, the processor fires the flash tube, otherwise it waits until there is adeguate charge. Any change in sampling rate is detectable at the flash count output channel.

During a flash, the currents produced from the PMT and reference detectors are used to pull down charged integration capacitors. The time to recharge each capacitor to a fixed voltage is a measure of the integrated light from that defector. Using comparators and internal timers, the digitized output from each detector is stored in an internal register. Prinr to each flash, the charge on the integration capacitors is adjusted for dark current effects. This effectively eliminates dark current noise from total detected light output. 
To determine the integrated pulse height above any background light, background must be subtracted from the total light detected. Background includes Rayleigh scattering by air, scattering by fine aerosol and stray scattering from the walls and dark trap. Since Rayleigh and aerosol background are continuously changing during balloon ascent, background must be continuously monitored. The aerosol sensor uses an algorithm for background correction that assumes most flashes contain background information only, with no large particles present. If this criterion is not met then coincidence counting is likely and concentrations are above the performance limit of the instrument. Background is measured with a moving exponential average over 50 flashes (2.5 seconds). If any pulse is more than twice present background, the increase in background is limited to 2\%. This provides a smoothly changing background that is relatively insensitive to large particles yet will quickly adjust to aerosol layers and chariging atmospheric pressure. Both the background average and total scattering (including coarsc aerosol and background) are available as digital Rs-232 or analoo oitputs. After background is subtracted, the remainder is divided by the reference signal to normalize the output to constant chamber illumination and then stored in a data register.

Four channels of size discrimination are available, with the channel edge values stored in memory registers. The background corrected signal is compared to these internal discriminator settings and a counter for the appropriate size range is incremented. The channel spacing is set with dip switches on the main circuit board. The channels are set such that the lower edge of channel 1 is $1 / 64$ that of the lower edge of channel 4. Channeis 2 and 3 are equal logarithmic spacing between 1 and 4 . Pulse height of a known scatterer was set by PMT high voltage gain. Particle free air is used as a zero reference and freon-12 $\left(\mathrm{CCl}_{2} \mathrm{~F}_{2}\right)$ is used for an 
upscale reference value. The lower edges of the four channels are approximately $5 \mathrm{um}, 13 \mathrm{um}, 26 \mathrm{um}$, and $50 \mathrm{um}$ effective particle diameter. The minimum size detectable is determined by the noise in the background measurement, that is the flash to flash variation rather than the average. The lower edge of channel 1 must be set to a voltage greater than the difference between the average background and the flash to flash noise, otherwise false counts will be produced in the first channel.

Real time data are available as seven: 0 to 5 volts, 16 bit resolution analog output channels. They include (1) total counts in each of 4 size channels, (2) the reference detector level, (3) total light scattering (analogous to a nephelometer) and (4) the incremental flash count. The size channels and flash count are incremental in $10 \mathrm{mv}$ and $5 \mathrm{mv}$ steps repectively. After the output ramp reaches 5.12 volts, ' that channel is reset to 0 volts and a new ramp is started. The reference detector and light scattering output are logarithmic at 1.5 volts/decade. The total light scattering channel is the total detected light without background suppression, including light scattered from any large particles (Figure 2 ). 


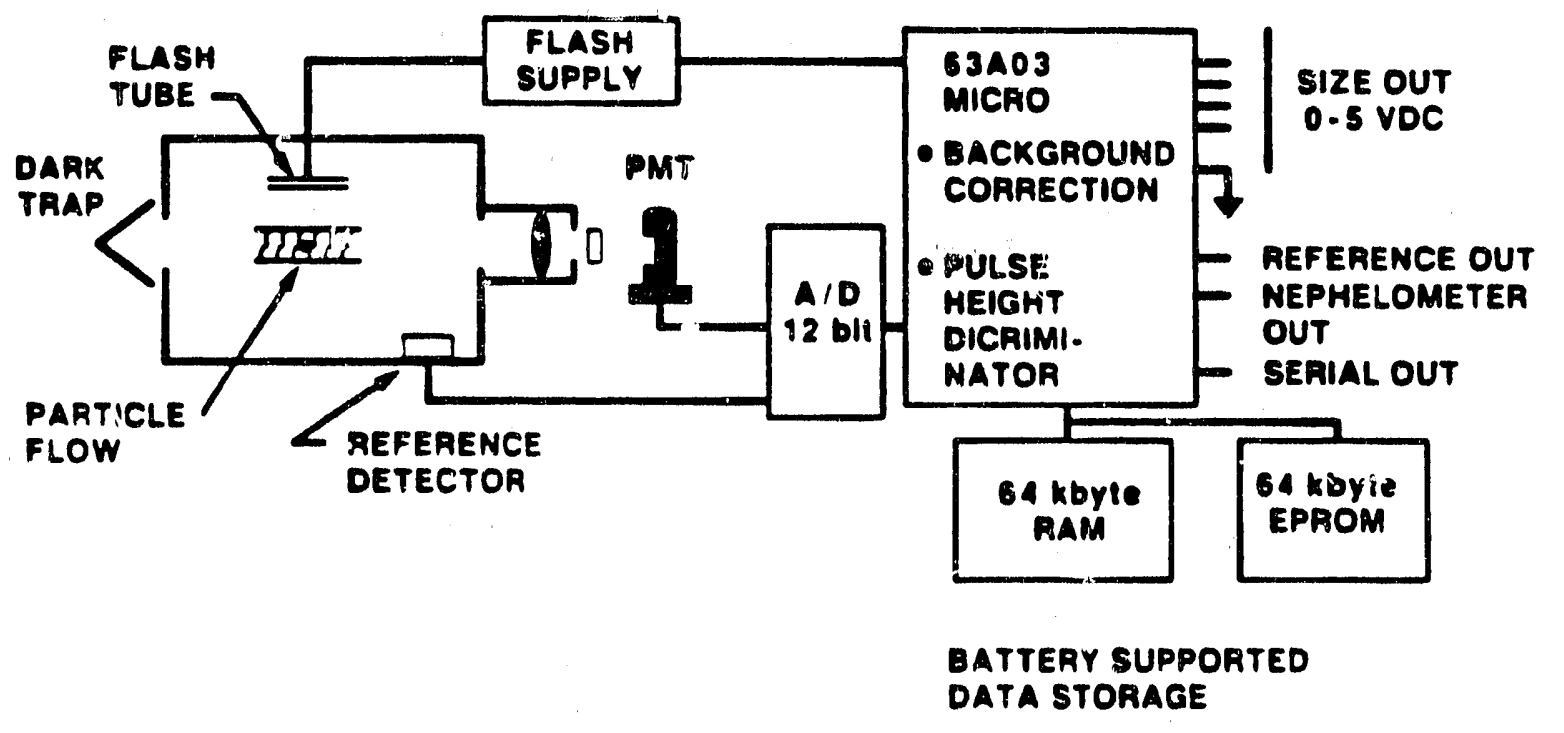

Figure 2. Aerosol spectrameter block diagram. 


\subsection{DATA MANDLING}

\subsection{Data Transmission}

The aerosol spectrometer uses a newly developed space Data Corporation (SDC) 16-channel voltage sonde for data transmission. standard metenrological parameters are measured by the SDC sonde, i.e.. relative humidity (carbon element hygristor), ambient temperature and pressure. Altitude is calculated using trigonometric techniques, with ranging, azimuth and elevation angles supplied by the SDC GMD-5 ground station. The sonde has 11 voltage input channels, with voltage input levels of 0 to 5 volts. Seven of these channels are used for transmission of aerosol spectrometer data. The voltages transmitted are flash count, size bin 1, size bin 2 , size bin 3, size bin 4, total light scatter and reference light level. The sonde converts all voltages to an audio frequency by means of a voltage controlled oscillator and uses this frequency to modulate a 9080 MHz carrier for transmission to the GMD-5 ground station.

\subsection{Data Processing}

The transmitted audio frequency is converted by the ground station software into the original voltages. After conversion, the meteorological parameters are reported in Etindard units. The aerosol spectrometer voltages are reported as raw voltages.

The flash count voltage is compared to the previous flash count valtage and the difference is divided by the flash count voltage inirement set in the aerosol spectrometer software (5 mi). The resultant value is the number of flashes since the last channel query. The number of flashes is multiplied by the scattering volume to give he total volume sampled between data trarismissions. 
The four size bin voltages are also compared to previous voltages. If the difference is zero, then no particles have passed through the scattering chamber since last channel query. If the difference is greater than zero, the voltage value is divided by the size bin voltage increment set in aerosol sensor software $(10 \mathrm{mv})$. The resultant value is the number of particles passing through the scattering volume since last channel cisery. This particle number divided by total sample volume (derived from flash count) gives particles per volume in each specified size bin.

Channel query occurs every 8 seconds on the SDC voltage sonde. Although the aerosol spectrometer is designed for use with the SDC sonde, at Air Force request, it is not limited to $*$ use with the SDC system. Any sonde with spare voltage input channels will suffice. Subsequent to its development, the aerosol spectrometer has been successfully integrated into an Atmospheric Instrumentation Research upper air sounding system. 
Instrument preparation and balloon flight operations were conducted by Air Force 6 th Weather squadron perscnnel of Hurlburt Field, Florida. Florida data were collected near Eglin AFB on five flights from March through July, 1988. California data were collected near Edwards AFB on four flights during August, 1988. Florida data are given in Appendix A and California data are given in Appendix B.

Significant coarse particle concentrations (greater than 10 particles/liter on consecutive data transmissions) are measured on only three of nine flights, i.e., March 22, April 15 and July 12. These concentrations are only seen in the Florida data.

\subsection{March 22,1988 Data Summary}

The balloon envelope was inilated to $10 \mathrm{~m}^{3}$ to acheive an ascent ratz of 5 to $7 \mathrm{~m} / \mathrm{s}$, giving a vertical resolution of 40 to $55 \mathrm{~m}$.

The aerosol spectrometer first detects particles at $9.9 \mathrm{~km}(287 \mathrm{mb})$ and last detects particles at $13.2 \mathrm{~km}$ $(178 \mathrm{mb})$, thus defining a particle cloud depth of $3.3 \mathrm{~km}$. The cloud top is situated at the base of the observed tropopause. Temperature measurements, made by onboard thermistor probe, show $-41{ }^{\circ} \mathrm{C}$ at the base of the cirrus layer, with decreasing temperature to $-65 \circ^{\circ} \mathrm{C}$ at the cloud top (Figure 3 ). Magono (2968) has concluded, from laboratory experiments, that large polycrystalline ice formation is initiated at temperatures above $-65{ }^{\circ} \mathrm{C}$. March 22 temperature measurements, within the cirrus cloud, are consistent with this observation. 
The data show four layers within the cirrus cloud (Figure 4). Concentrations as measured by the aerosol spectrometer are shown over approximate altitude increments in Table 1.

Particle size distribution within the first three layers (bases located at $9.9 \mathrm{~km}, 10.9 \mathrm{~km}$ and $11.8 \mathrm{~km}$ respectively) is roughly Gaussian. The fourth and final layer (base located at $12.5 \mathrm{~km}$ ) shows a marked skewing in particle size toward the smallest size bin of 5 to $13 \mathrm{um}$ (Figure 5). Less than 18 of the particles comprising the upper two layers are larger than 26 um. Particle size distribution within the cirrus cloud layers are shown in Table 2. Particle concentrations and size distributions are similar to those measured by aircraft instrumentation and reported by Heymsfield (1986). 


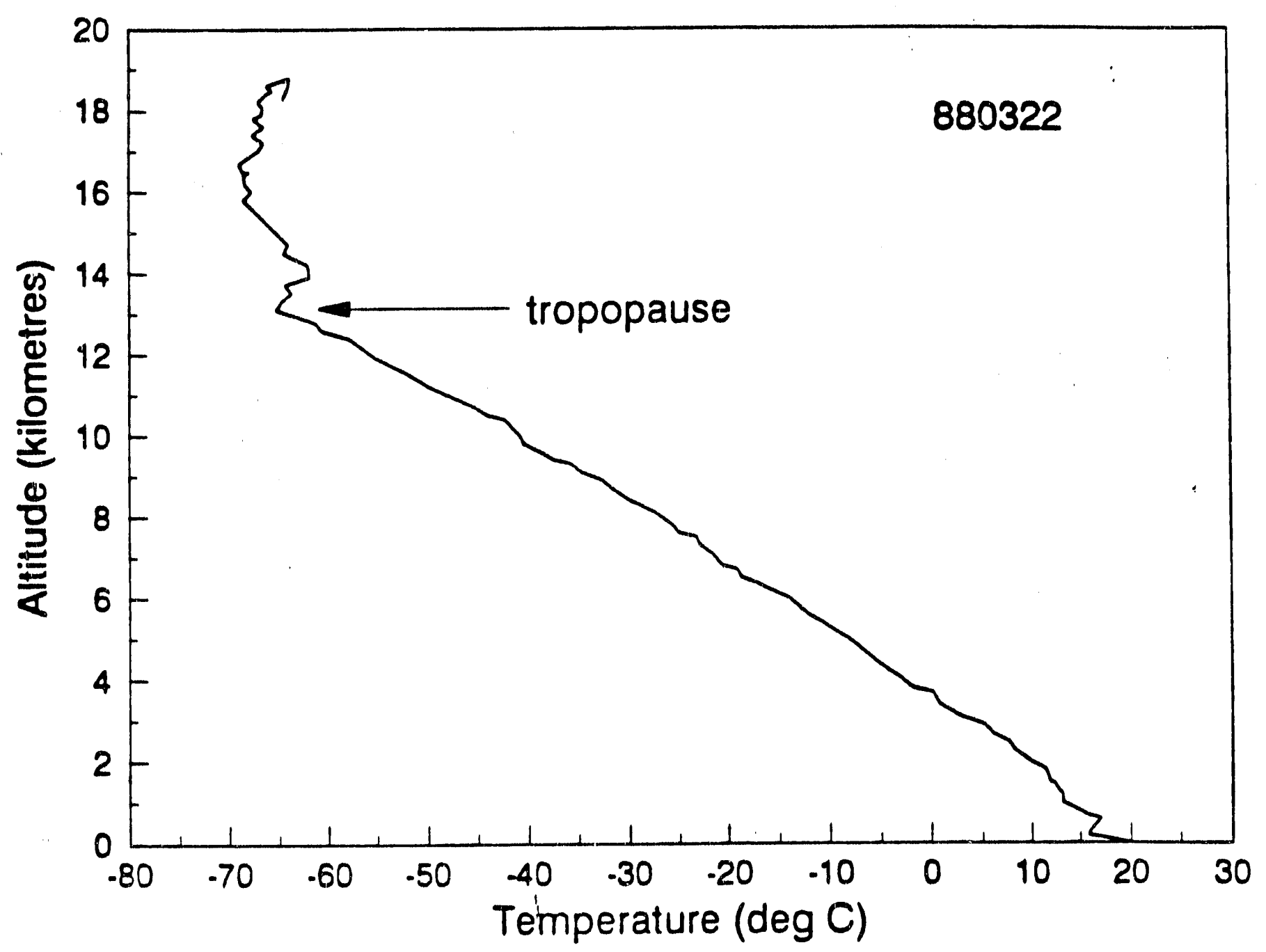

Figure 3. Vertical temperature proflle March 22, 1988. 


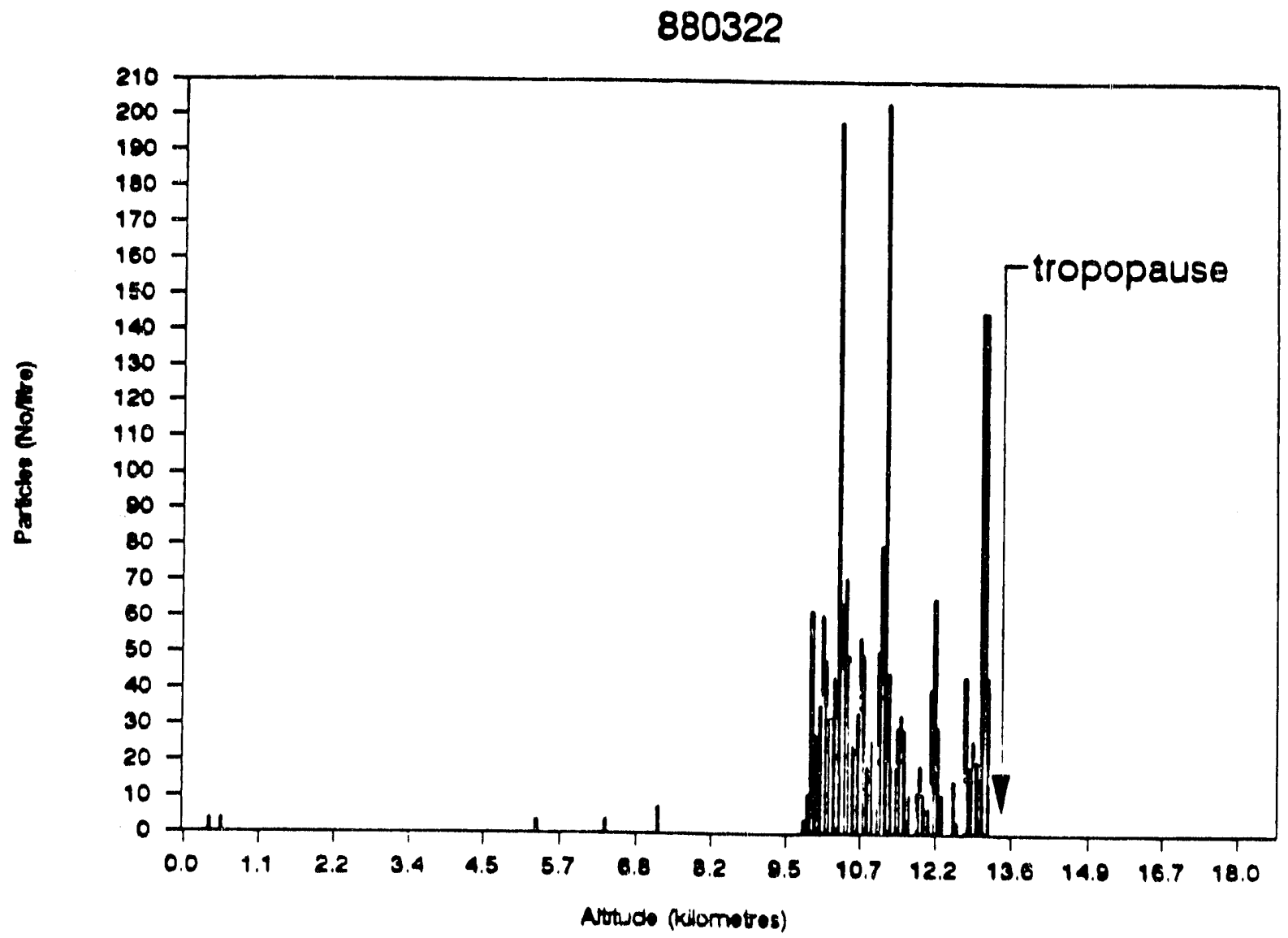

Figure 4. Particle concentration distribution March 22, 1988. 


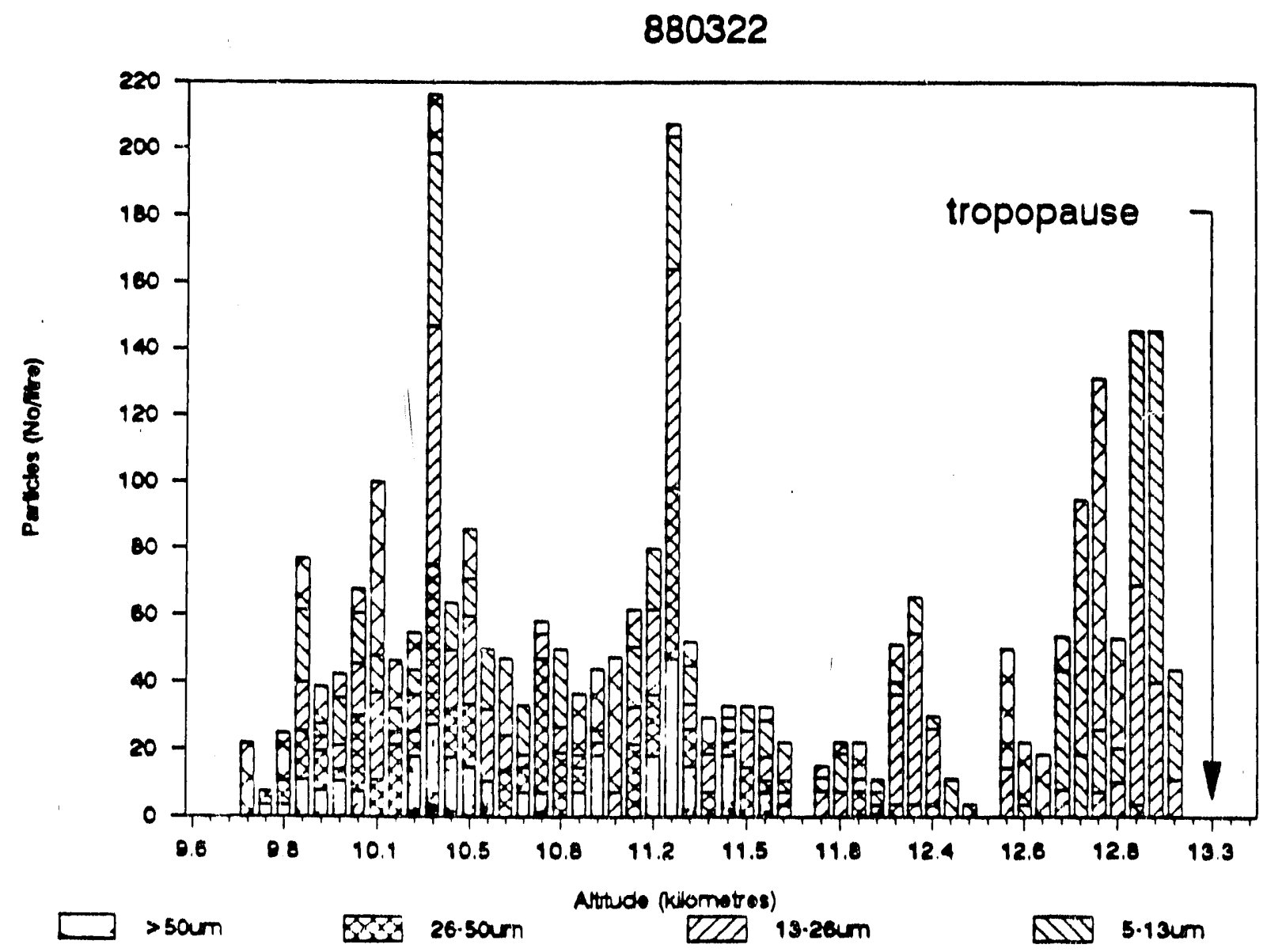

Figure 5. Particle size distribution March 22, 1988. 


\subsection{Apr11 15,2988 pate Eumary}

Particles are first detected at an altitude of $12.6 \mathrm{~km}$ (181 mb) and last detected at $12.9 \mathrm{~km}(175 \mathrm{mb})$, defining a particje cloud depth of $0.3 \mathrm{~km}$. The cloud top is again situated at the base of the observed tropopause (Figure 6). Temperature at the base of the cloud $18-58.6$ OC with $-62.00^{\circ} \mathrm{C}$ at the cloud top (Figure 7).

The one shallow layer shows a particle size distribution skewed toward the 5 to 13 um size bin (Figure 8). The particle size distribution is remarkably similar to the fourth layer of the March 22 cloud, indicating similar ice crystal growth conditions near the tropopause.

\subsection{Iuly 12,2988 pata Bummary}

The aerosol spectrometer first detects particles at $11.9 \mathrm{~km}(213 \mathrm{mb})$ and last detects particles at $14.3 \mathrm{~km}$ (142 mb), defining the particle cloud depth of $2.4 \mathrm{~km}$. The cloud top is situated at the base of the observed tropopause. The temperature at the cloud base is $-46.6{ }^{\circ} \mathrm{C}$ and drops to -66.6 oc at the cloud top (Figure 9).

The data show four distinct layers within the cirrus cloud, just as in the March 22 data (Figure 10). All layers exhibit a Gaussian distribution of particle concentration with altitude.

Particle size distribution within the inaividual layers is also similar to the March data. Very few large particles are seen in any layer (Figure 11). The size distribution is skewed toward particles with less than 26 um effective diameter in the upper two layers, as shown in Table 3 . 
880415

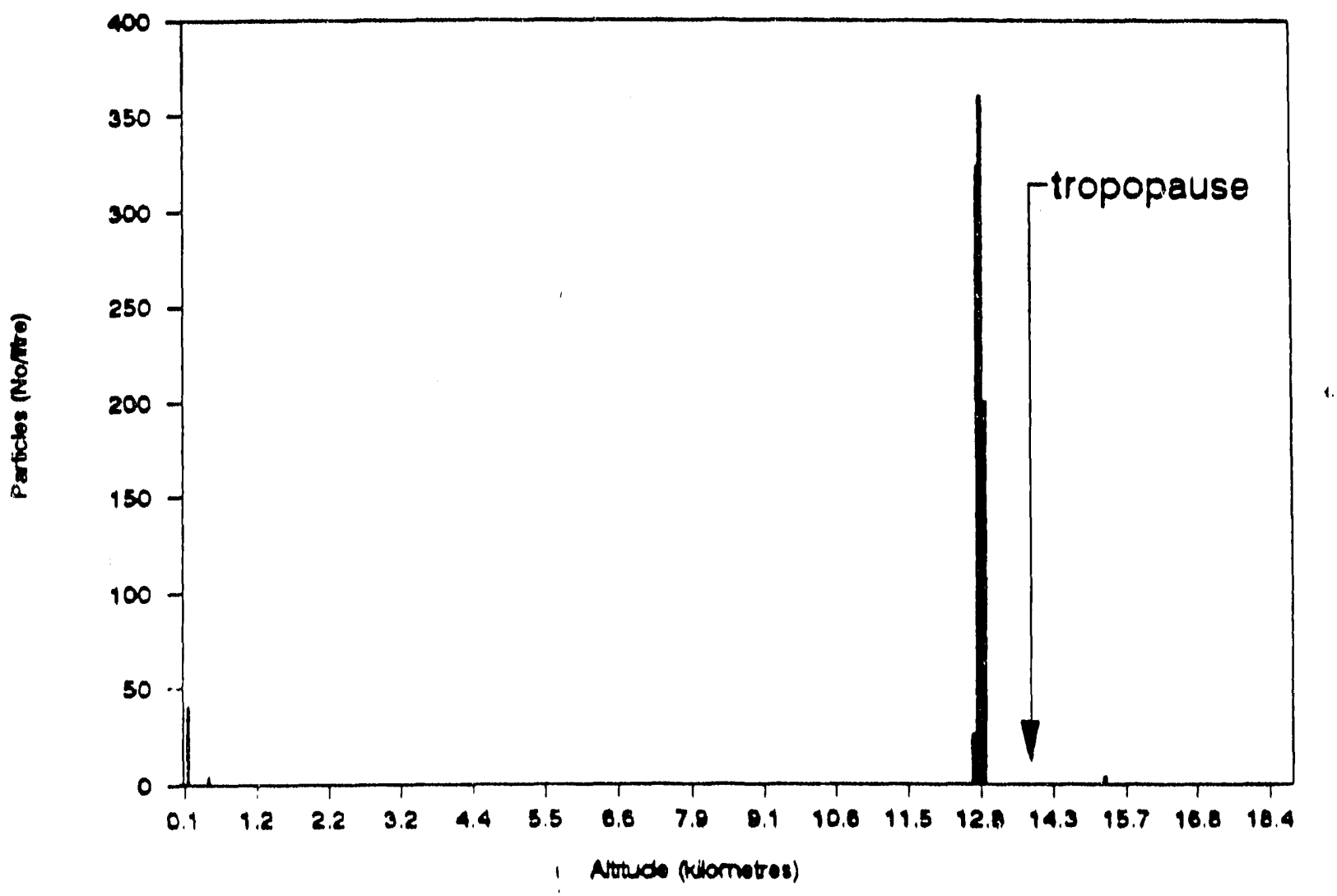

Figure 6. Particle concentration distribution April 15, 1988. 


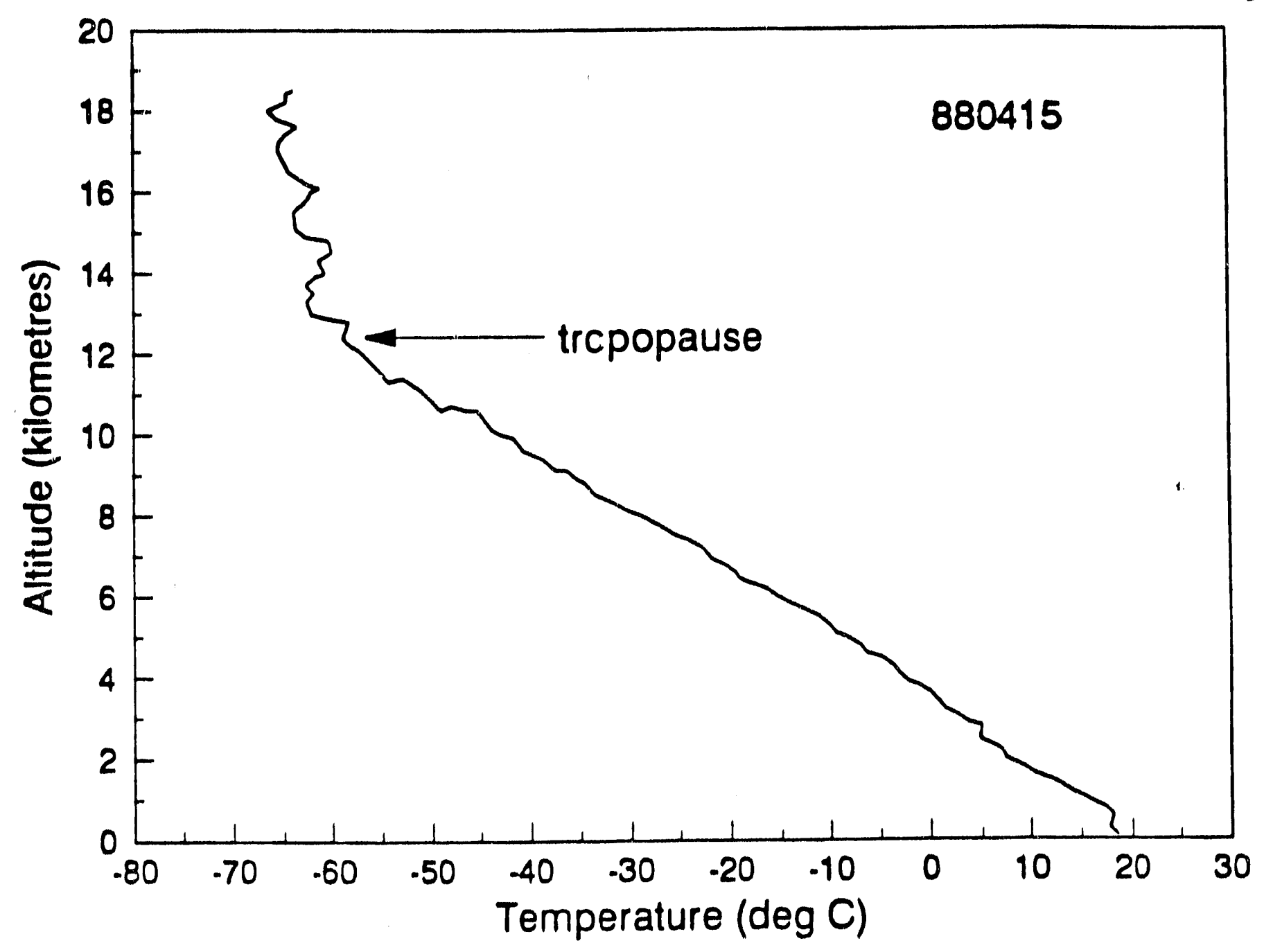

Figure 7. Vertical temperature proflle April 15, 1988. 


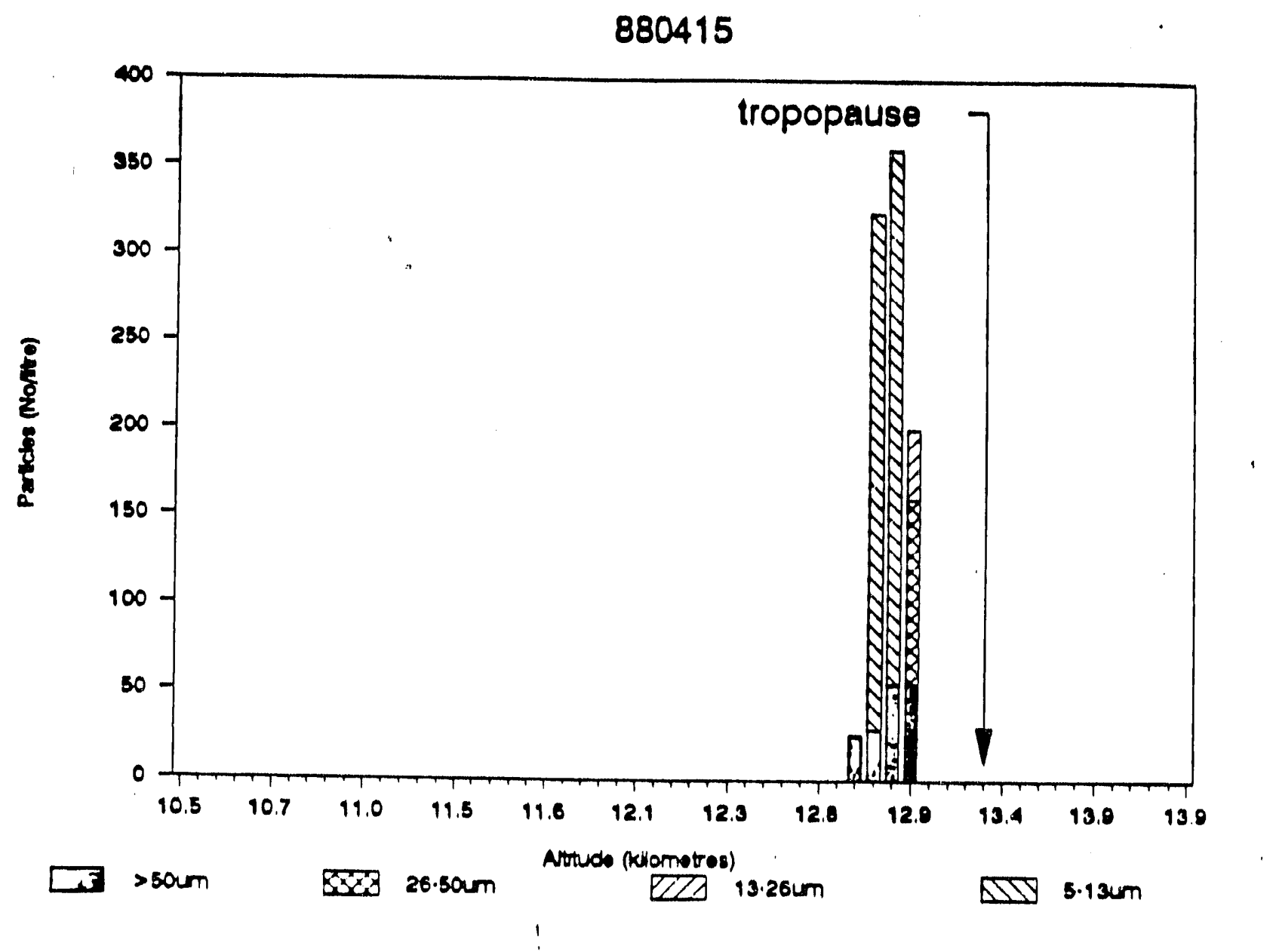

Figure B. Particle size distribution April 15, 1988. 


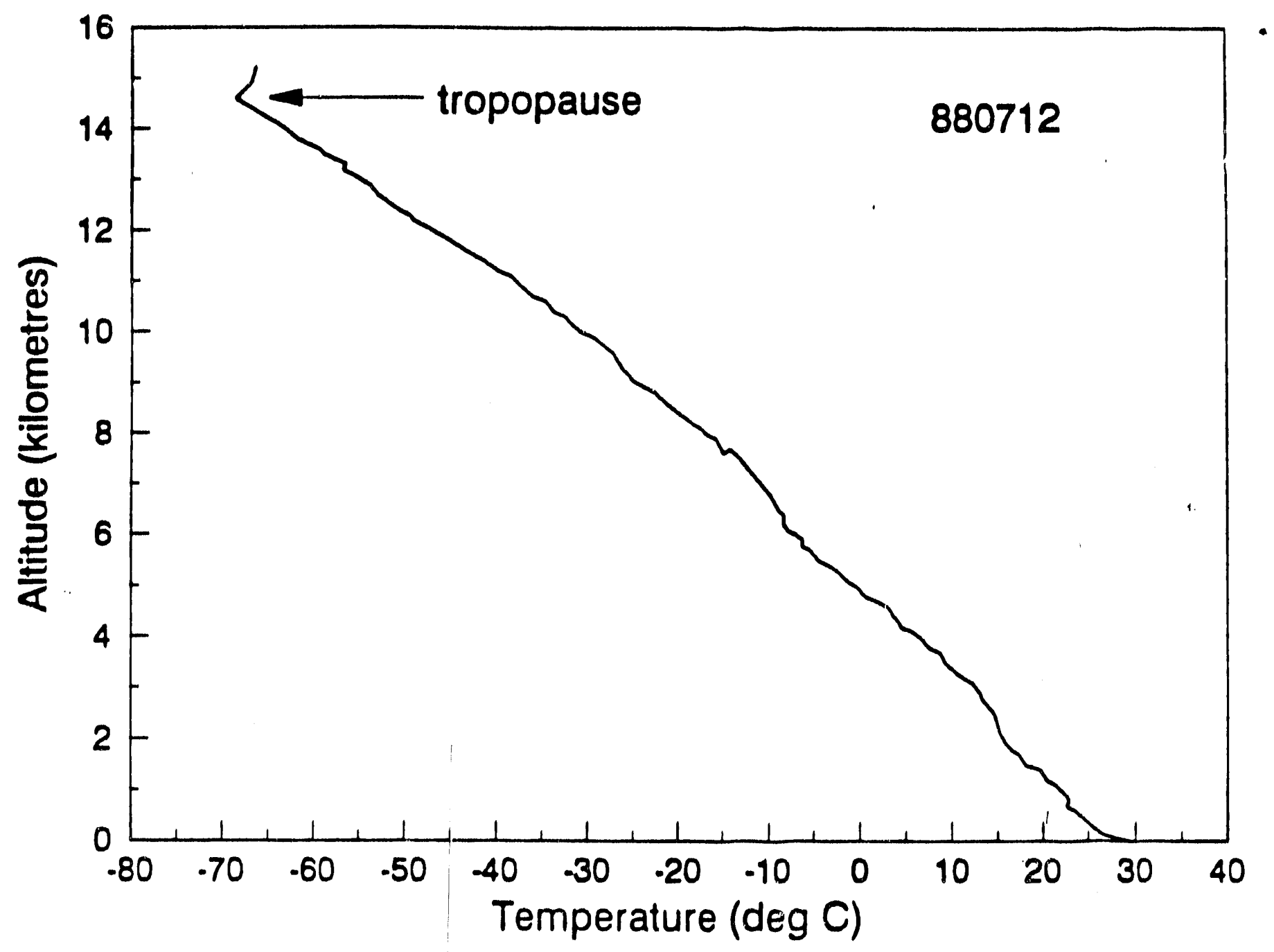

Figure 9. Vertical temperature profile July 12, 1988. 


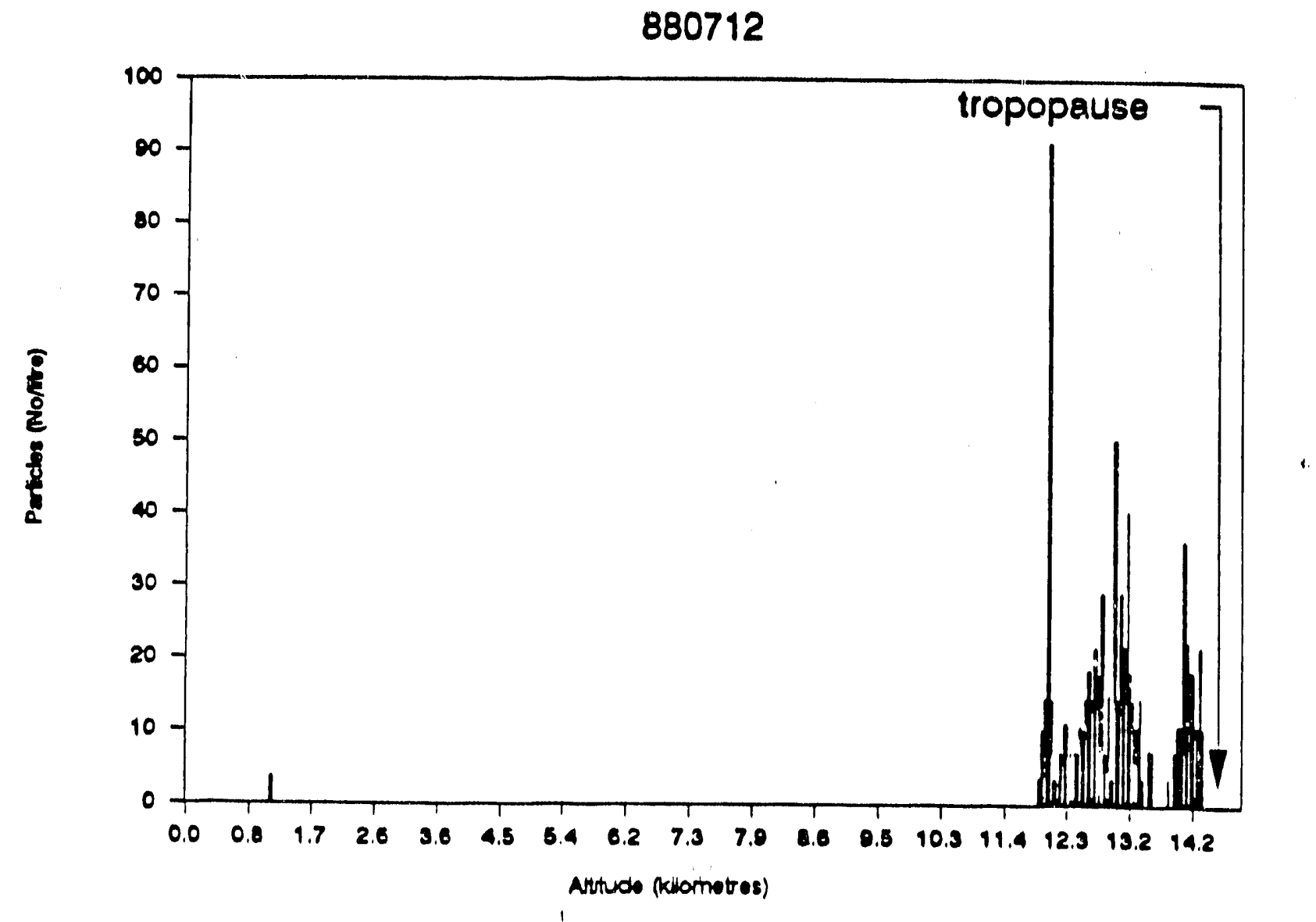

Figure 10. Particle concentration distribution July 12, 1988. 


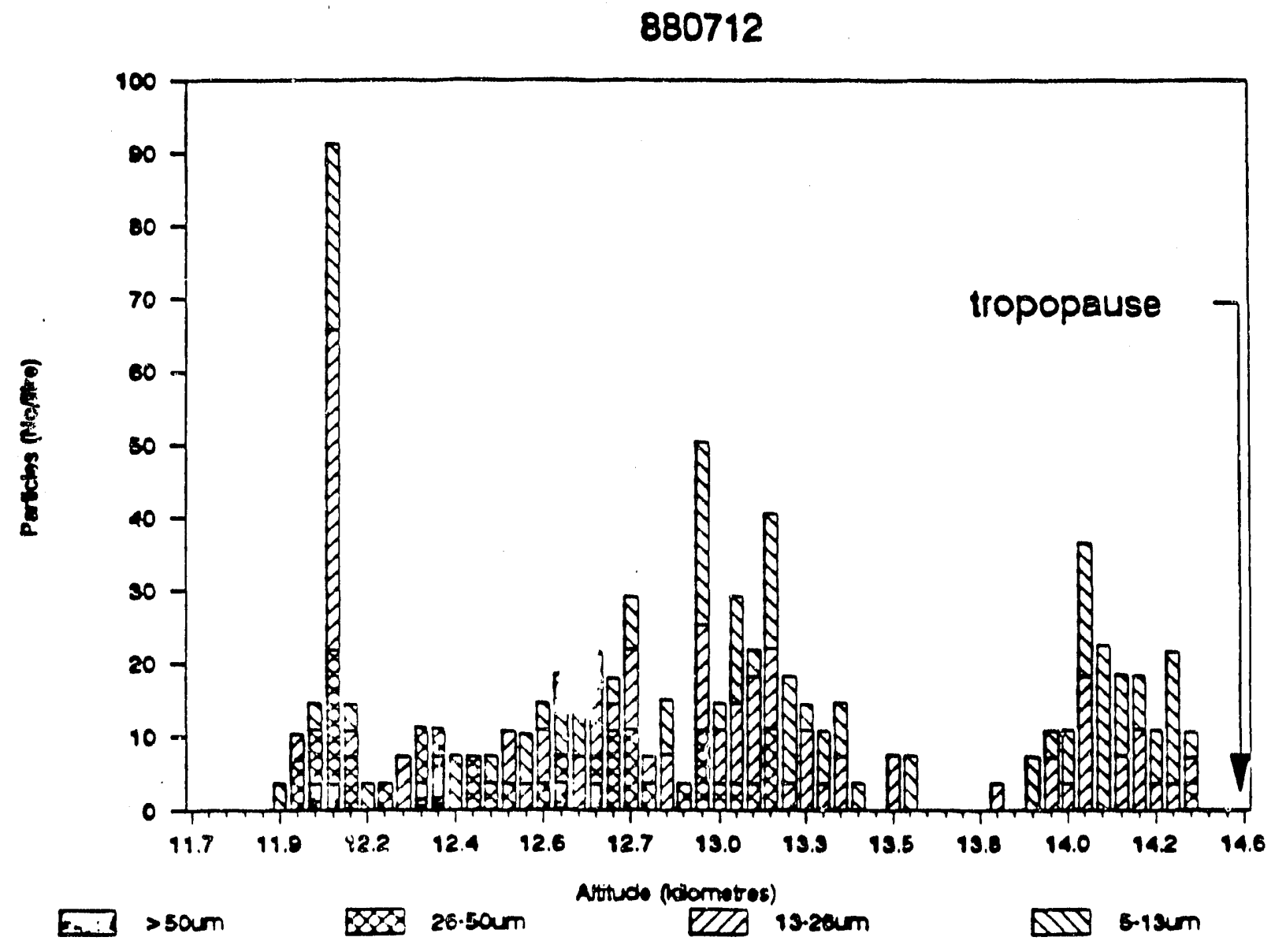

Figure 11. Particle size distribution July 12, 1988. 
Table 1. Concentration Distribution March 22, 1988.

\begin{tabular}{|c|c|c|}
\hline $\begin{array}{c}\text { Altitude } \\
\text { (kilometers) }\end{array}$ & $\begin{array}{c}\text { Concentration } \\
\text { (liters) }\end{array}$ & Layer \\
\hline $\begin{array}{r}9.9 \\
10.0 \\
10.1 \\
10.3 \\
10.4 \\
10.5 \\
10.7 \\
10.8\end{array}$ & $\begin{array}{l}91 \\
97 \\
80 \\
242 \\
135 \\
74 \\
87 \\
68\end{array}$ & I \\
\hline $\begin{array}{l}10.9 \\
11.1 \\
11.3 \\
11.4 \\
11.5 \\
11.7\end{array}$ & $\begin{array}{l}34 \\
130 \\
232 \\
63 \\
41 \\
23\end{array}$ & II \\
\hline $\begin{array}{l}11.9 \\
12.1 \\
12.4\end{array}$ & $\begin{array}{l}19 \\
207 \\
42\end{array}$ & III \\
\hline $\begin{array}{l}12.5 \\
12.6 \\
12.7 \\
12.8 \\
13.1\end{array}$ & $\begin{array}{l}18 \\
43 \\
44 \\
165 \\
189\end{array}$ & IV \\
\hline
\end{tabular}


Table 2. Particle Size Relative Frequency March 22,1988. Layer I.

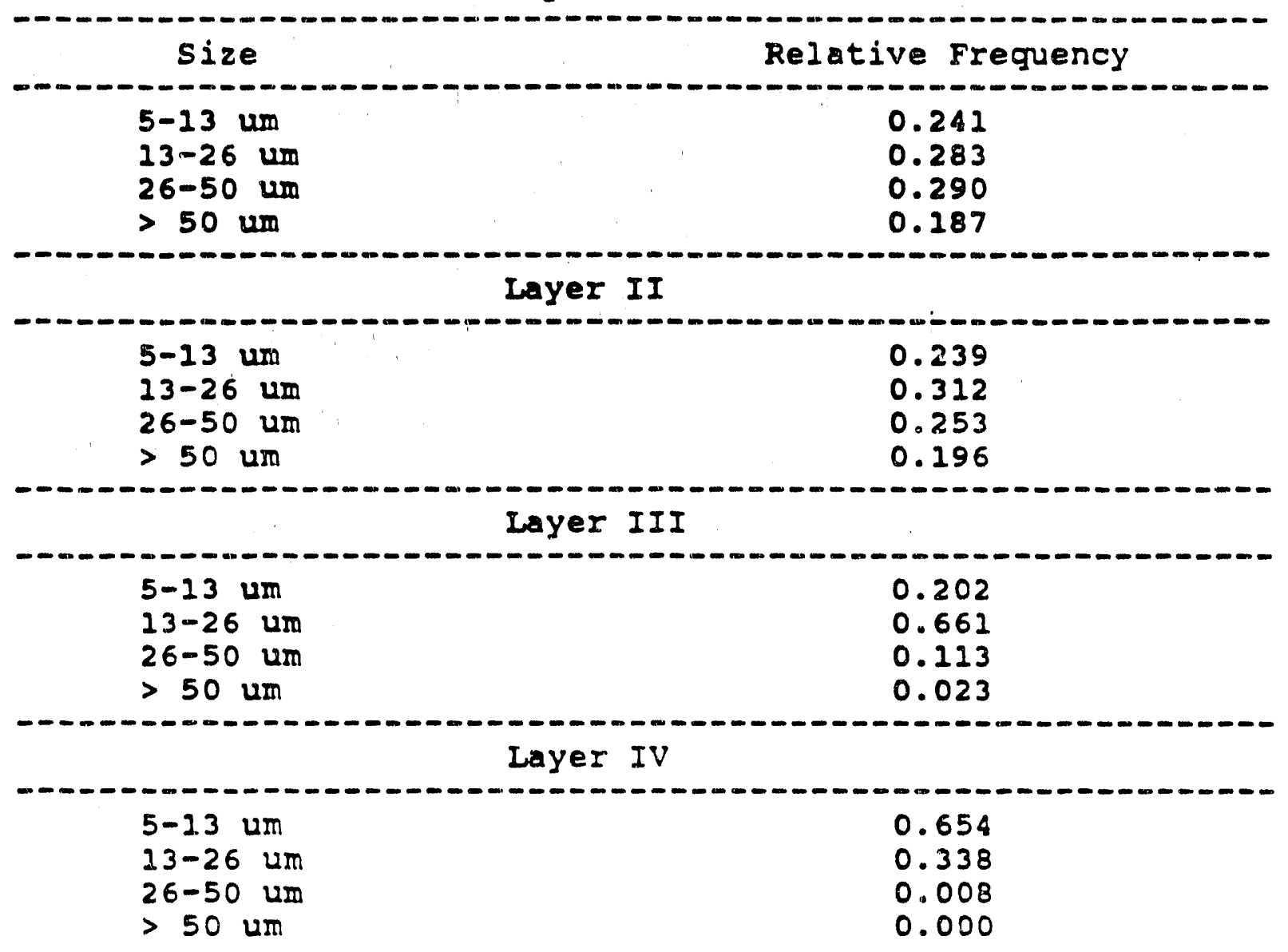


Table 3. Particle size Relative Frequency July 12,1988.

\section{Isyer I}

$\begin{array}{ll}\text { Size } & \text { Relative Frequency } \\ 5-13 \mathrm{um} & 0.279 \\ 23-26 \mathrm{um} & 0.375 \\ 26-50 \mathrm{um} & 0.287 \\ >50 \mathrm{um} & 0.059 \\ & \\ 5-13 \mathrm{um} & 0.267 \\ 13-26 \mathrm{um} & 0.327 \\ 26-50 \mathrm{um} & 0.318 \\ 50 \mathrm{um} & 0.088\end{array}$

Layer ITI

$\begin{array}{ll}5-13 \mathrm{um} & 0.460 \\ 13-26 \mathrm{um} & 0.381 \\ 26-50 \mathrm{um} & 0.159 \\ >50 \mathrm{um} & 0.000\end{array}$

\section{Layer IV}

$\begin{array}{ll}5-13 \text { um } & 0.596 \\ 13-26 \text { um } & 0.381 \\ 26-50 \mathrm{um} & 0.023 \\ >50 \mathrm{um} & 0.000\end{array}$




\subsection{CONCLOSION}

The Sandia/Radiance developed high altitude balloon-borne aerosol spectrometer shows much promise as a useful tool for the characterization of high thin cirrus clouds. With the advent of portable tracking stations, frequent soundings from virtually anywhere in the world are possible. The balioon-borne aerosol spectrometer offers a cost effective alternative to aircraft based high altitude coarse particle measurement programs. 


\section{REFERENCES}

Heymsfield, A. J., 1986. "Ice Particles Observed in Cirriform Cloud at $-83 R C$ and Implications for Polar stratospheric

Clouds," J. Atmos. Sci., 43:851-855.

Magono, C., 1968. "On the Additional Nucleation of Natural snow Crystals," J. Rech. Atmos., 3:147-152. 


\section{APPENDIX A}

FLORIDA DATA 
2 2MAR8 8

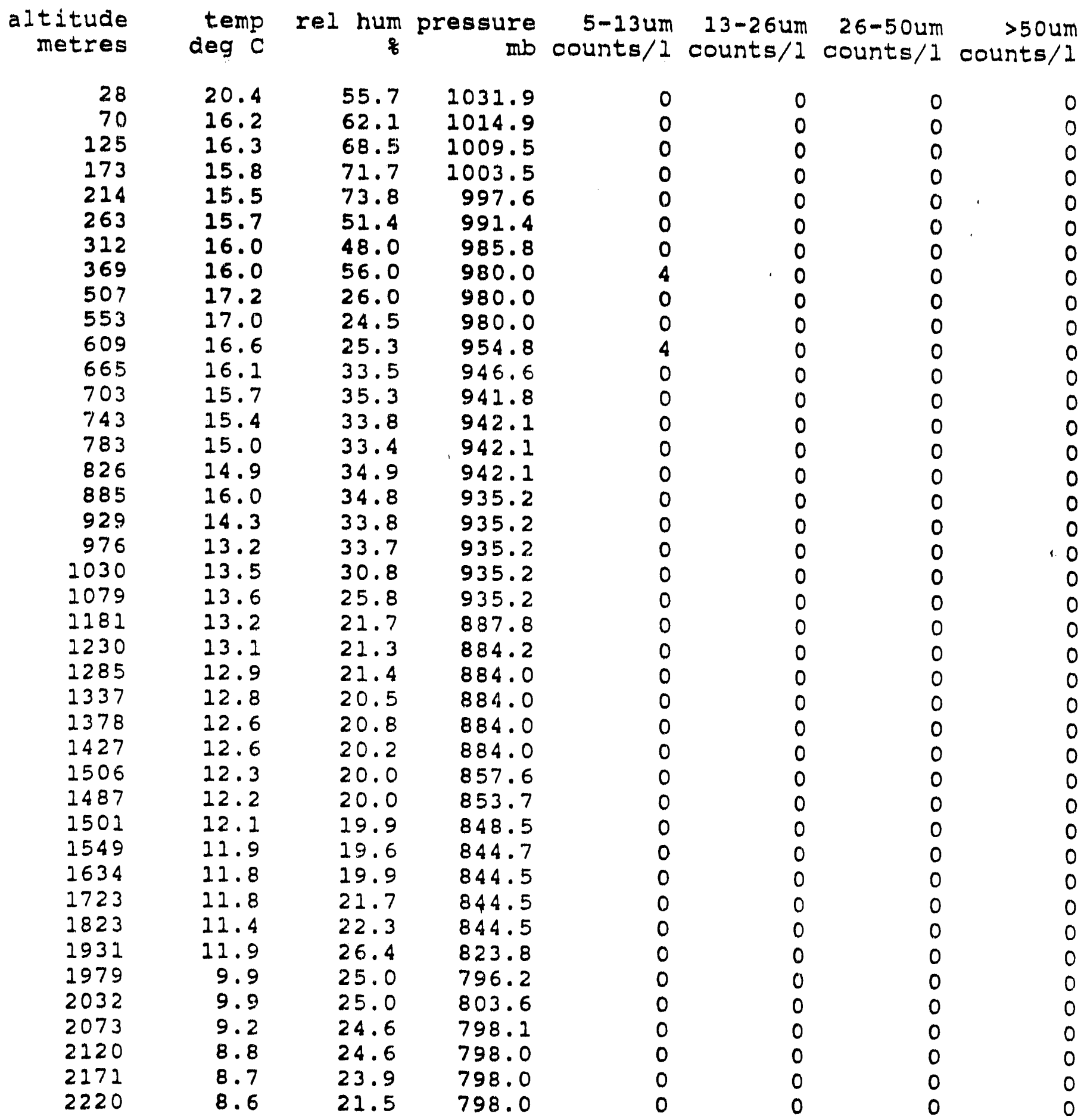




\section{MAR8 8}

altitude metres

2263

2308

2368

2410

2497

2601

2649

2701

2754

2800

2857

2902

2945

3000

3043

3091

3144

3198

3346

3388

3450

3491

3537

3581

3630

3677

3737

3792

3833

3888

3938

4078

4130

4177

4231

4273

4327

4370

4423

4480

4528 temp rel hum pressure deg $C$

\section{5}

8.3

8.1

7.9

7.7

8.0

6.6

6.1

5.7

5.3

5.3

4.8

4.4

4.1

3.6

3.3

3.0

2.9

1.7

0.8

1.1

0.7

0.5

0.3

$-0.2$

0.2

$-0.9$

$-1.5$

$-1.9$

$-2.4$

$-2.8$

$-3.4$

$-3.7$

$-2.5$

$-4.1$

$-4.1$

$-4.4$

$-5.2$

$-5.3$

$-5.7$

$-5.7$
21.0

20.2

19.0

19.4

20.1

21.2

27.4

20.4

20.8

20.8

20.5

20.9

21.6

22.5

25.5

22.5

22.3

21.8

21.4

20.8

20.4

20.5

19.9

19.8

20.1

27.9

21.6

21.9

22.2

22.7

27.7

20.2

20.3

18.9

19.7

19.5

19.0

19.2

19.1

19.5

19.4 mb counts/1 counts/1 counts/1

$>50 \mathrm{um}$ counts / I

$\begin{array}{ll}798.0 & 0 \\ 798.0 & 0 \\ 769.6 & 0 \\ 765.2 & 0 \\ 761.0 & 0 \\ 757.9 & 0 \\ 757.9 & 0 \\ 757.9 & 0 \\ 757.9 & 0 \\ 757.9 & 0 \\ 728.6 & 0 \\ 723.1 & 0 \\ 719.9 & 0 \\ 714.9 & 0 \\ 714.1 & 0 \\ 711.1 & 0 \\ 711.1 & 0 \\ 711.1 & 0 \\ 684.8 & 0 \\ 681.1 & 0 \\ 676.3 & 0 \\ 676.3 & 0 \\ 676.3 & 0 \\ 676.3 & 0 \\ 676.3 & 0 \\ 676.3 & 0 \\ 650.9 & 0 \\ 647.5 & 0 \\ 642.3 & 0 \\ 642.4 & 0 \\ 642.4 & 0 \\ 642.4 & 0 \\ 617.4 & 0 \\ 625.9 & 0 \\ 609.5 & 0 \\ 609.5 & 0 \\ 609.5 & 0 \\ 602.1 & 0 \\ 602.1 & 0 \\ 602.1 & 0 \\ 602.1 & \\ & 0\end{array}$

0
0
0
0
0
0
0
0
0
0
0
0
0
0
0
0
0
0
0
0
0
0
0
0
0
0
0
0
0
0
0
0
0
0
0
0
0
0
0
0
0

0
0
0
0
0
0
0
0
0
0
0
0
0
0
0
0
0
0
0
0
0
0
0
0
0
0
0
0
0
0
0
0
0
0
0
0
0
0
0
0
0

0 
22MARB B

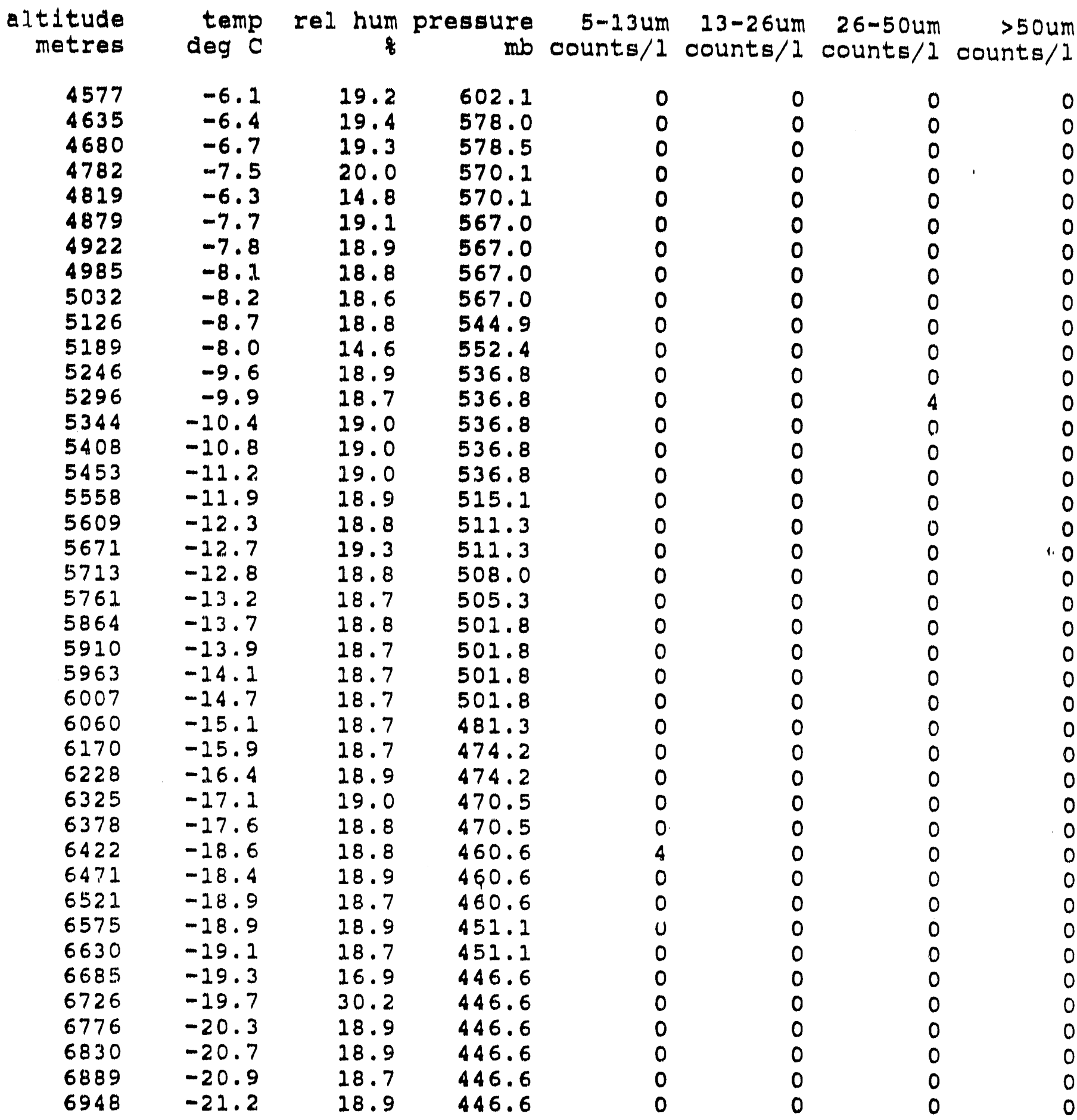




\section{MAR8 8}

altitude temp rel hum pressure 5-13um 13-26um 26-50um >50um metres deg $c$

mb counts/1 counts/1 counts/1 counts/1

\begin{tabular}{|c|c|c|c|c|}
\hline $\begin{array}{l}7118 \\
7174 \\
7240 \\
7290 \\
7349 \\
7408 \\
7454 \\
7506 \\
7559 \\
7611 \\
7658 \\
7709 \\
7752 \\
7933 \\
7988 \\
8051 \\
8106 \\
8212 \\
8271 \\
8307 \\
8364 \\
8408 \\
8531 \\
8587 \\
8711 \\
8768 \\
8825 \\
8880 \\
8979 \\
9042 \\
9082 \\
9135 \\
9189 \\
9258 \\
9310 \\
9348 \\
9400 \\
9521 \\
9574 \\
9606 \\
9655\end{array}$ & $\begin{array}{l}-21.8 \\
-22.9 \\
-22.6 \\
-23.0 \\
-23.4 \\
-23.6 \\
-23.4 \\
-24.3 \\
-24.7 \\
-25.1 \\
-25.4 \\
-25.7 \\
-25.8 \\
-26.5 \\
-27.5 \\
-27.4 \\
-28.2 \\
-28.6 \\
-29.1 \\
-29.0 \\
-29.2 \\
-30.0 \\
-30.6 \\
-31.0 \\
-31.9 \\
-32.3 \\
-32.8 \\
-32.8 \\
-33.9 \\
-34.3 \\
-34.8 \\
-35.4 \\
-35.7 \\
-35.8 \\
-36.7 \\
-36.7 \\
-37.5 \\
-38.4 \\
-38.7 \\
-38.8 \\
-39.3\end{array}$ & $\begin{array}{l}18.8 \\
18.8 \\
18.8 \\
18.7 \\
18.9 \\
18.9 \\
16.8 \\
18.9 \\
19.1 \\
19.0 \\
19.2 \\
19.3 \\
19.3 \\
19.5 \\
19.4 \\
19.5 \\
19.7 \\
19.8 \\
19.7 \\
19.6 \\
15.5 \\
19.7 \\
19.7 \\
18.2 \\
19.8 \\
19.6 \\
20.0 \\
19.8 \\
19.9 \\
19.8 \\
19.7 \\
19.9 \\
19.9 \\
19.9 \\
19.9 \\
20.0 \\
20.3 \\
20.1 \\
20.5 \\
20.4 \\
20.4\end{array}$ & $\begin{array}{l}419.4 \\
416.8 \\
416.8 \\
412.8 \\
412.8 \\
406.7 \\
414.9 \\
41.4 .9 \\
414.9 \\
414.9 \\
414.9 \\
379.9 \\
383.4 \\
378.0 \\
378.0 \\
378.0 \\
378.0 \\
359.5 \\
356.3 \\
354.1 \\
354.5 \\
351.0 \\
348.1 \\
348.1 \\
348.1 \\
348.1 \\
328.9 \\
323.7 \\
321.7 \\
319.9 \\
320.8 \\
316.1 \\
315.2 \\
311.7 \\
310.6 \\
307.2 \\
305.9 \\
300.0 \\
296.3 \\
295.7 \\
293.5\end{array}$ & $\begin{array}{l}0 \\
0 \\
0 \\
0 \\
0 \\
0 \\
0 \\
0 \\
0 \\
0 \\
0 \\
0 \\
0 \\
0 \\
0 \\
0 \\
0 \\
0 \\
0 \\
0 \\
0 \\
0 \\
0 \\
0 \\
0 \\
0 \\
0 \\
0 \\
0 \\
0 \\
0 \\
0 \\
0 \\
0 \\
0 \\
0 \\
0 \\
0 \\
0 \\
0 \\
0\end{array}$ \\
\hline
\end{tabular}

$\begin{array}{lll}0 & 0 & 0 \\ 0 & 0 & 0 \\ 0 & 0 & 0 \\ 7 & 0 & 0 \\ 0 & 0 & 0 \\ 0 & 0 & 0 \\ 0 & 0 & 0 \\ 0 & 0 & 0 \\ 0 & 0 & 0 \\ 0 & 0 & 0 \\ 0 & 0 & 0 \\ 0 & 0 & 0 \\ 0 & 0 & 0 \\ 0 & 0 & 0 \\ 0 & 0 & 0 \\ 0 & 0 & 0 \\ 0 & 0 & 0 \\ 0 & 0 & 0 \\ 0 & 0 & 0 \\ 0 & 0 & 0 \\ 0 & 0 & 0 \\ 0 & 0 & 0 \\ 0 & 0 & 0 \\ 0 & 0 & 0 \\ 0 & 0 & 0 \\ 0 & 0 & 0 \\ 0 & 0 & 0 \\ 0 & 0 & 0 \\ 0 & 0 & 0 \\ 0 & 0 & 0 \\ 0 & 0 & 0 \\ 0 & 0 & 0 \\ 0 & 0 & 0 \\ 0 & 0 & 0 \\ 0 & 0 & 0 \\ 0 & 0 & 0 \\ 0 & 0 & 0 \\ 0 & 0 & 0 \\ 0 & 0 & 0 \\ 0 & 0 & 0 \\ 0 & 0 & 0\end{array}$


22MAR8 8

altitude

temp rel hum pressure

5-13um 13-26um 26-50um $>50 u m$ metres deg $C$

mb counts/1 counts/1 counts/1 counts/1

$\begin{array}{llll}9730 & -39.9 & 20.4 & 291.0 \\ 9779 & -40.5 & 20.7 & 287.3 \\ 9828 & -40.9 & 21.2 & 285.9 \\ 9890 & -41.2 & 22.0 & 283.4 \\ 10023 & -40.9 & 15.8 & 283.9 \\ 10061 & -41.3 & 29.2 & 278.5 \\ 10110 & -41.2 & 33.2 & 277.8 \\ 10138 & -41.3 & 32.5 & 272.8 \\ 10209 & -42.1 & 39.6 & 272.8 \\ 10346 & -42.3 & 37.2 & 266.6 \\ 10393 & -42.4 & 38.3 & 263.3 \\ 10421 & -42.9 & 39.0 & 262.3 \\ 10511 & -43.6 & 39.3 & 262.3 \\ 10537 & -44.1 & 39.6 & 257.9 \\ 10625 & -44.6 & 40.1 & 257.8 \\ 10717 & -45.0 & 40.2 & 257.8 \\ 10736 & -45.4 & 40.4 & 257.8 \\ 10798 & -46.1 & 41.0 & 257.8 \\ 10896 & -46.8 & 41.7 & 257.8 \\ 10911 & -47.2 & 42.2 & 243.3 \\ 10998 & -47.8 & 42.4 & 237.2 \\ 11130 & -48.8 & 42.2 & 235.4 \\ 11233 & -49.8 & 42.2 & 231.7 \\ 11280 & -50.7 & 42.4 & 230.3 \\ 11342 & -50.6 & 42.4 & 227.8 \\ 11388 & -51.2 & 42.4 & 226.9 \\ 11463 & -51.5 & 42.5 & 227.4 \\ 11521 & -52.4 & 42.6 & 222.7 \\ 11585 & -52.5 & 42.7 & 222.7 \\ 11771 & -53.2 & 42.8 & 218.5 \\ 11738 & -53.3 & 42.8 & 216.1 \\ 11768 & -54.1 & 42.8 & 214.3 \\ 11806 & -54.4 & 43.0 & 211.1 \\ 11894 & -55.0 & 42.9 & 210.1 \\ 11923 & -55.1 & 42.8 & 208.1 \\ 12140 & -56.8 & 42.9 & 201.2 \\ 12176 & -57.5 & 42.9 & 199.0 \\ 12437 & -57.9 & 42.9 & 196.8 \\ 12310 & -58.7 & 42.8 & 194.4 \\ 12360 & -59.1 & 42.9 & 192.9 \\ 12450 & -59.5 & 42.8 & 190.5\end{array}$

$\begin{array}{rr}0 & 0 \\ 0 & 0 \\ 0 & 0 \\ 22 & 15 \\ 4 & 4 \\ 14 & 7 \\ 15 & 15 \\ 11 & 26 \\ 7 & 4 \\ 7 & 11 \\ 52 & 71 \\ 14 & 18 \\ 11 & 26 \\ 18 & 21 \\ 0 & 10 \\ 15 & 4 \\ 0 & 7 \\ 23 & 8 \\ 0 & 4 \\ 4 & 4 \\ 0 & 7 \\ 18 & 11 \\ 18 & 25 \\ 40 & 65 \\ 11 & 7 \\ 0 & 11 \\ 4 & 4 \\ 7 & 11 \\ 11 & 7 \\ 4 & 4 \\ 0 & 0 \\ 4 & 8 \\ 11 & 7 \\ 0 & 0 \\ 4 & 0 \\ 4 & 0 \\ 11 & 0 \\ 4 & 0 \\ 11 & 0 \\ 0 & 0 \\ 0 & \end{array}$

$\begin{array}{rr}0 & 0 \\ 0 & 4 \\ 7 & 4 \\ 15 & 11 \\ 12 & 8 \\ 4 & 11 \\ 23 & 8 \\ 11 & 0 \\ 21 & 0 \\ 7 & 18 \\ 48 & 28 \\ 14 & 18 \\ 19 & 15 \\ 0 & 11 \\ 14 & 0 \\ 7 & 7 \\ 40 & 7 \\ 19 & 0 \\ 7 & 7 \\ 0 & 19 \\ 0 & 0 \\ 22 & 0 \\ 18 & 18 \\ 51 & 47 \\ 11 & 15 \\ 7 & 0 \\ 4 & 18 \\ 15 & 0 \\ 4 & 7 \\ 4 & 0 \\ 0 & 0 \\ 0 & 0 \\ 0 & 0 \\ 7 & 0 \\ 0 & 0 \\ 4 & 0 \\ 4 & 0 \\ 4 & 0 \\ 0 & 0 \\ 0 & 0 \\ 0 & 0\end{array}$


22 MARB 8

altitude metres

$\begin{array}{llll}12527 & -59.7 & 42.9 & 190.4 \\ 12564 & -60.2 & 42.9 & 189.3 \\ 12567 & -60.6 & 43.0 & 187.9 \\ 12686 & -60.8 & 40.8 & 188.2 \\ 12672 & -60.9 & 43.0 & 184.9 \\ 12761 & -61.4 & 43.0 & 183.5 \\ 12790 & -61.9 & 43.0 & 182.0 \\ 13023 & -64.2 & 41.3 & 178.4 \\ 13114 & -65.2 & 43.2 & 178.4 \\ 13210 & -65.6 & 46.1 & 178.4 \\ 13256 & -65.5 & 43.2 & 168.8 \\ 13302 & -64.7 & 44.7 & 168.8 \\ 13410 & -64.4 & 43.1 & 165.8 \\ 13380 & -63.5 & 42.4 & 164.8 \\ 13471 & -63.7 & 42.9 & 164.8 \\ 13559 & -64.0 & 42.8 & 161.8 \\ 13614 & -64.0 & 42.7 & 161.8 \\ 13663 & -64.3 & 42.6 & 158.7 \\ 13775 & -64.4 & 42.5 & 154.9 \\ 13897 & -62.8 & 42.2 & 153.8 \\ 13936 & -61.9 & 42.1 & 149.2 \\ 13952 & -62.6 & 42.0 & 151.2 \\ 14059 & -63.0 & 41.6 & 147.9 \\ 14154 & -62.1 & 41.4 & 146.0 \\ 14218 & -63.2 & 41.2 & 145.0 \\ 14346 & -62.9 & 41.2 & 142.4 \\ 14437 & -63.9 & 41.0 & 142.1 \\ 14412 & -63.9 & 41.0 & 139.8 \\ 14440 & -64.4 & 40.9 & 140.0 \\ 14502 & -64.5 & 40.9 & 136.7 \\ 14558 & -64.9 & 40.8 & 137.7 \\ 14610 & -64.6 & 40.7 & 135.2 \\ 14711 & -64.1 & 40.6 & 133.1 \\ 14781 & -64.0 & 40.5 & 132.1 \\ 14830 & -64.5 & 40.6 & 130.2 \\ 14857 & -64.9 & 40.4 & 129.2 \\ 15560 & -67.5 & 40.3 & 113.2 \\ 15668 & -68.2 & 40.2 & 115.1 \\ 15783 & -68.6 & 40.2 & 111.2 \\ 15884 & -67.8 & 40.2 & 110.2 \\ 15933 & -68.9 & 40.1 & 108.7\end{array}$

if

\& temp rel hum pressure mb counts/1 counts/1 counts/1 counts/1

$\operatorname{deg} C$

5-13um

13-26um

\section{0}

0
4
35

14
0
0
8
0

18

19

10

76

105

33

0

0

0

0

0

0

0

0

0

0

0

0

0

0

0

0

0

0

0
0
0

0

0

0

0

0

0

0

10

65

40

0

0

0

0

0

0

0

0

0
0

0

0

0

0

c

0
0

0

0
0

0

0

0

0

0
0
0
0
0
0
0
0
0
0
4
0
0
0
0
0
0
0
0
0
0
0
0
0
0
0
0
0
0
0
0
0
0
0
0
0
0
0
0
0
0
0
0
0

0

0

0 


\section{MAR8 8}

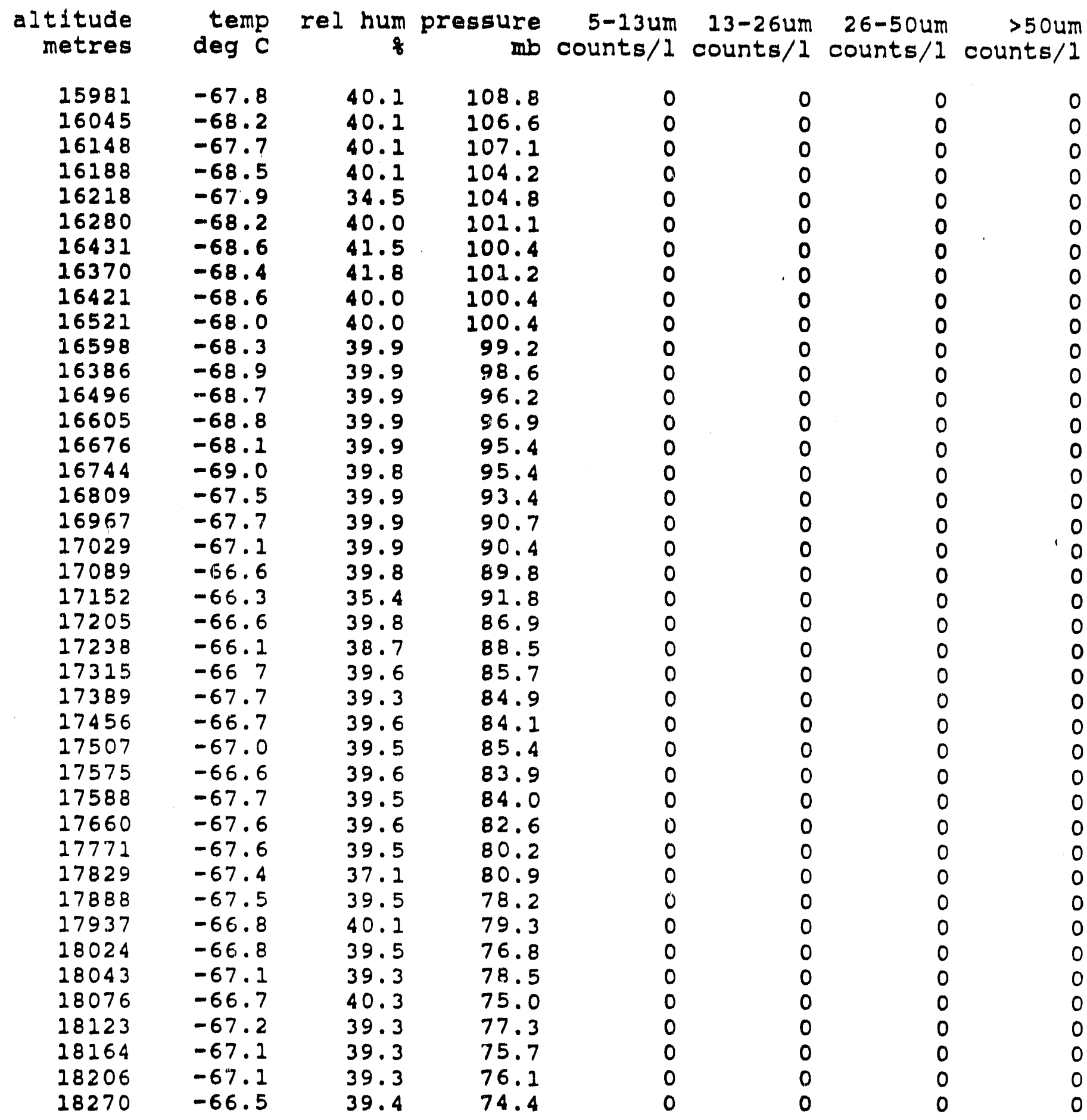




\section{MAR8 8}

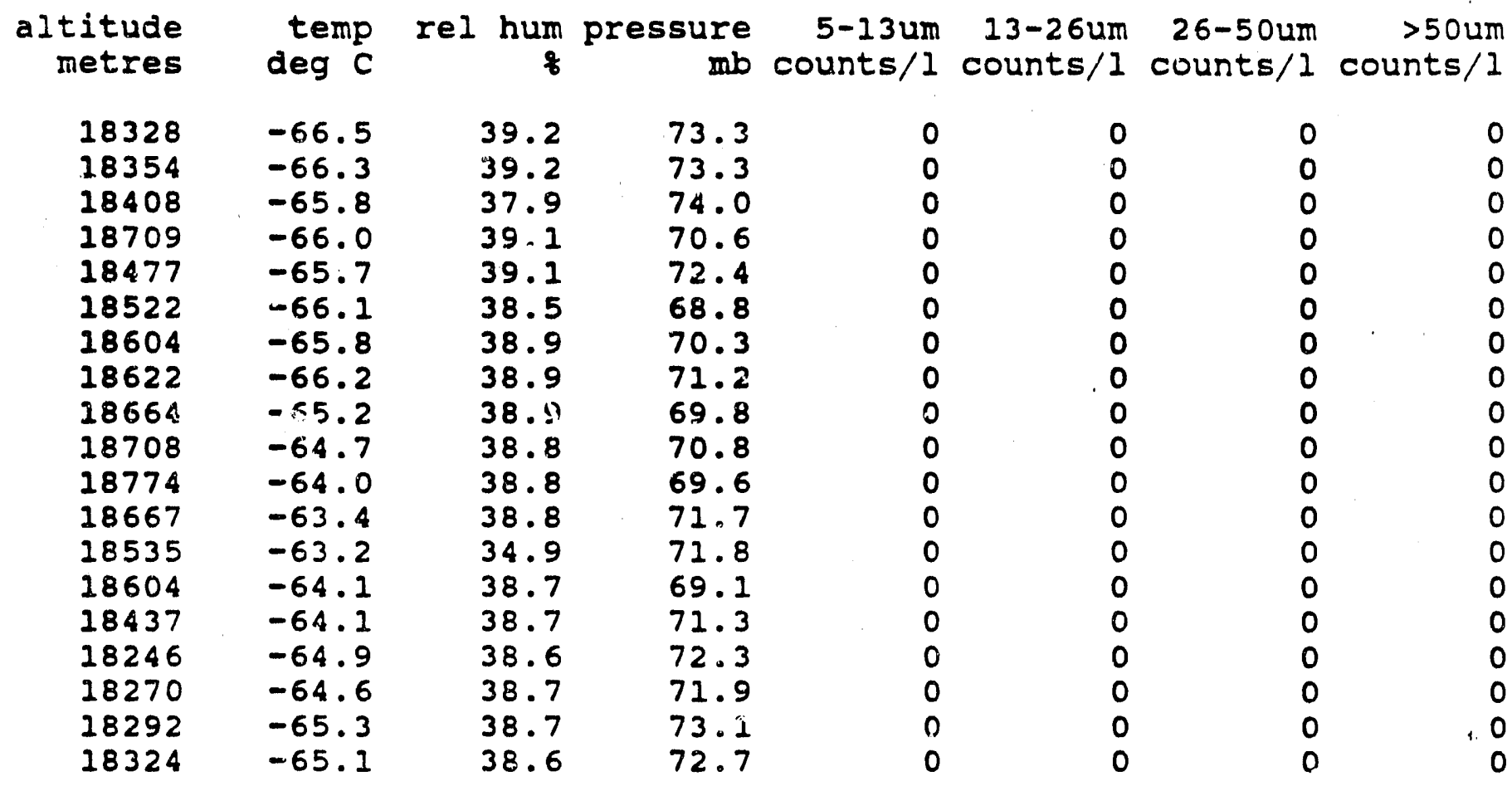




\section{APR8 8}

altitude metres

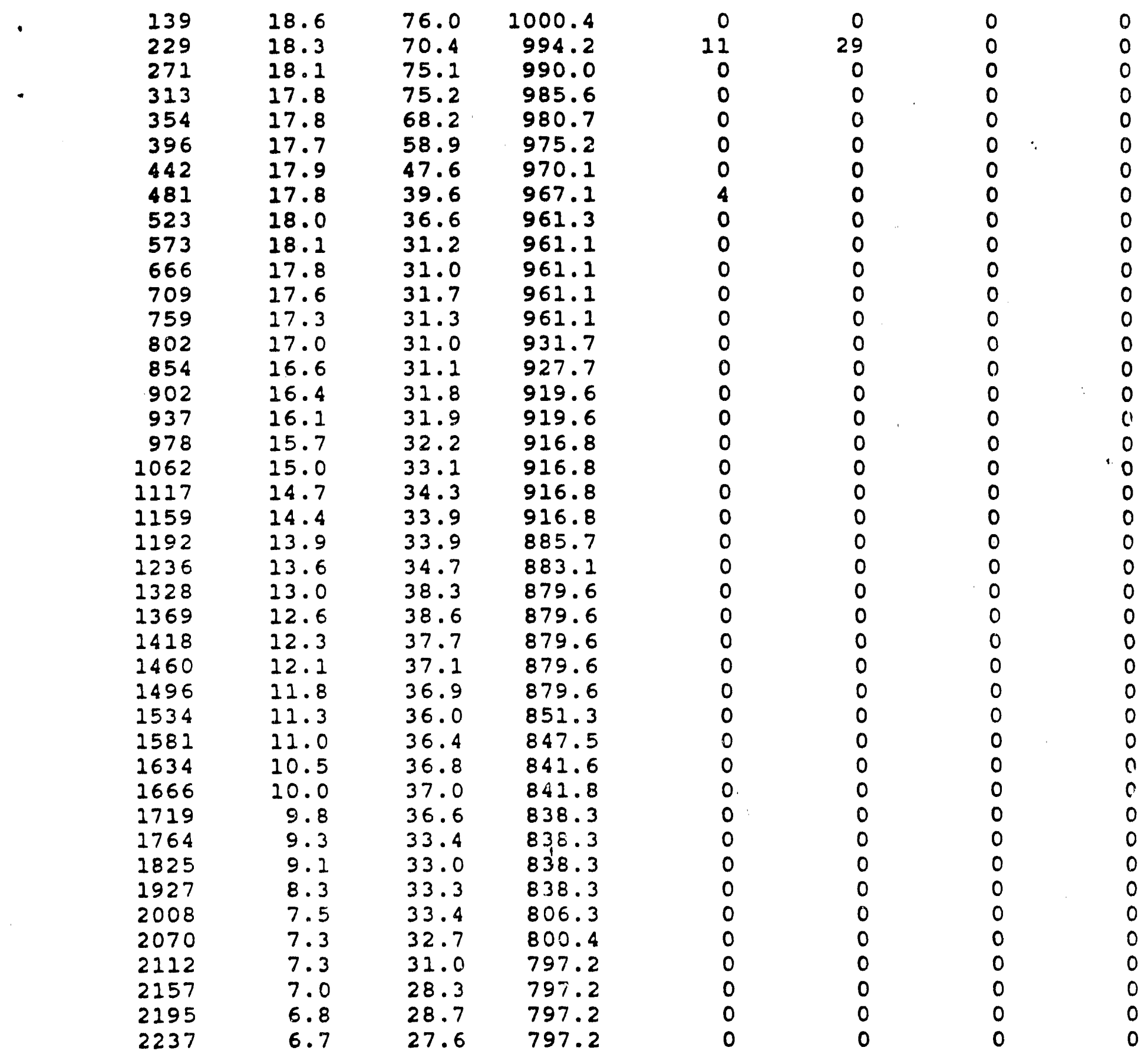

$\begin{array}{rrrrr}\text { temp rel hum pressure } 5-13 u m & 13-26 u m & 26-50 u m & >50 u m \\ \operatorname{deg} C & \text { mb counts/1 counts } / 1 \text { counts } / 1 \text { counts } / 1\end{array}$ $\begin{array}{rrrrr}\text { temp rel hum pressure } 5-13 u m & 13-26 u m & 26-50 u m & >50 u m \\ \operatorname{deg} C & \text { mb counts/1 counts } / 1 \text { counts } / 1 \text { counts } / 1\end{array}$ 
15APR8 8

altitude metres

$\begin{array}{lrll}2275 & 6.5 & 27.4 & 797.2 \\ 2309 & 6.0 & 27.2 & 797.2 \\ 2376 & 5.7 & 26.0 & 772.8 \\ 2403 & 5.5 & 26.3 & 768.7 \\ 2465 & 5.2 & 26.3 & 763.5 \\ 2492 & 4.9 & 26.2 & 761.1 \\ 2536 & 4.8 & 25.9 & 757.5 \\ 2666 & 4.9 & 21.3 & 757.5 \\ 2716 & 4.8 & 20.7 & 757.5 \\ 2766 & 4.9 & 20.8 & 757.5 \\ 2814 & 4.5 & 20.8 & 734.4 \\ 2860 & 4.2 & 21.3 & 727.6 \\ 2904 & 3.7 & 25.6 & 724.0 \\ 2950 & 3.3 & 28.4 & 721.1 \\ 3000 & 3.0 & 28.9 & 721.1 \\ 3058 & 2.5 & 30.0 & 721.1 \\ 3105 & 2.1 & 30.5 & 721.1 \\ 3152 & 1.8 & 30.9 & 721.1 \\ 3202 & 1.5 & 30.9 & 721.1 \\ 3257 & 1.1 & 31.2 & 688.2 \\ 3409 & 0.6 & 25.9 & 680.8 \\ 3457 & 0.4 & 25.2 & 680.8 \\ 3505 & 0.5 & 25.9 & 680.8 \\ 3545 & 0.3 & 25.6 & 680.8 \\ 3588 & 0.1 & 25.6 & 680.8 \\ 3688 & -0.3 & 25.5 & 654.9 \\ 3731 & -0.7 & 25.6 & 651.5 \\ 3776 & -1.2 & 26.3 & 647.1 \\ 3825 & -1.7 & 27.3 & 646.9 \\ 3870 & -1.9 & 27.4 & 644.4 \\ 3908 & -2.3 & 28.1 & 644.4 \\ 3945 & -2.6 & 28.2 & 644.4 \\ 3996 & -2.8 & 27.3 & 644.4 \\ 4115 & -3.2 & 26.3 & 620.8 \\ 4145 & -3.4 & 24.8 & 613.1 \\ 4215 & -3.4 & 23.4 & 611.8 \\ 4280 & -3.8 & 24.1 & 611.6 \\ 4357 & -4.5 & 24.2 & 607.4 \\ 4414 & -4.7 & 25.4 & 607.4 \\ 4485 & -4.9 & 25.7 & 597.6 \\ 4524 & -5.3 & 25.8 & 597.6 \\ 4543 & -5.7 & 25.5 & 597.6\end{array}$
temp rel hum pressure $5-13$ um $13-26 u m$ 26-50um $>50 u m$
deg C temp rel hum pressure $5-13 u m$ 13-26um $26-50 u m$ in $>50 u m$
deg C $\begin{array}{rrrr}\text { temp rel hum pressure } 5-13 u m & 13-26 u m & 26-50 u m & >50 u m \\ \text { deg C } & \text { mb counts/1 counts/1 counts/1 counts } / 1\end{array}$

0

0

0

0

0

0

0

0

0

0

0

0

0

0

0

0

0

0

0

0

0

0

0

0

0

0

0
0

0
0

0
0

0

0

0

0

0
0
0

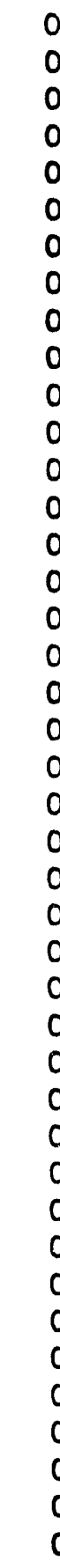

0

0

0

0

0

0

0

0

0

0

. 0

0

0
0

0

0

0

0

0

0

0

0

0

0

0

0

0

0

0

0

0

0

0 
15APR8 8

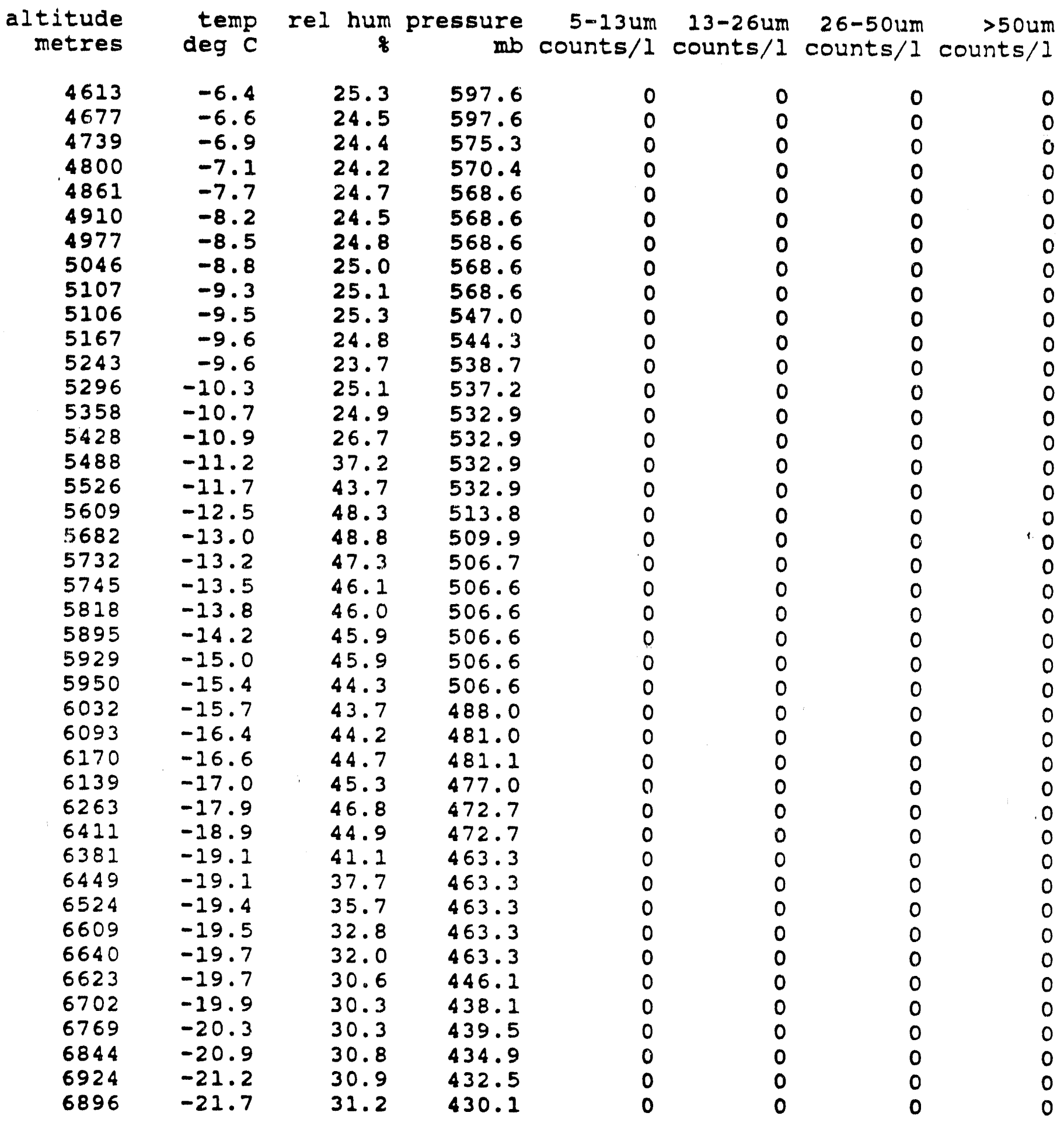


15APK 88

altitude metres

$\begin{array}{lllll}6925 & -21.9 & 31.8 & 430.0 & 0 \\ 7166 & -22.4 & 29.4 & 421.6 & 0 \\ 7255 & -22.7 & 29.3 & 421.6 & 0 \\ 7188 & -22.9 & 29.1 & 421.6 & 0 \\ 7242 & -23.3 & 29.0 & 411.9 & 0 \\ 7331 & -23.8 & 29.0 & 411.9 & 0 \\ 7400 & -24.3 & 29.2 & 404.3 & 0 \\ 7485 & -24.5 & 29.3 & 404.3 & 0 \\ 7551 & -25.2 & 29.7 & 404.3 & 0 \\ 7483 & -25.5 & 30.0 & 404.3 & 0 \\ 7581 & -26.1 & 30.6 & 404.3 & 0 \\ 7672 & -26.5 & 30.7 & 389.0 & 0 \\ 7736 & -26.9 & 31.3 & 384.7 & 0 \\ 7832 & -27.4 & 31.5 & 383.7 & 0 \\ 7902 & -28.5 & 33.0 & 379.7 & 0 \\ 7983 & -29.1 & 33.0 & 379.7 & 0 \\ 8173 & -29.5 & 33.5 & 370.0 & 0 \\ 8150 & -29.9 & 33.5 & 370.0 & 0 \\ 8076 & -30.3 & 33.6 & 370.0 & 0 \\ 8148 & -30.7 & 33.8 & 362.1 & 0 \\ 8243 & -31.1 & 33.9 & 362.1 & 0 \\ 8319 & -31.7 & 34.5 & 358.1 & 0 \\ 8419 & -32.6 & 34.9 & 358.1 & 0 \\ 8509 & -33.2 & 35.3 & 358.1 & 0 \\ 8485 & -33.6 & 35.8 & 348.2 & 0 \\ 8501 & -33.9 & 36.3 & 348.2 & 0 \\ 8597 & -34.1 & 35.9 & 348.2 & 0 \\ 8794 & -34.7 & 34.2 & 341.7 & 0 \\ 8670 & -35.0 & 33.9 & 341.7 & 0 \\ 8772 & -35.4 & 33.8 & 333.4 & 0 \\ 8870 & -35.5 & 33.4 & 333.4 & 0 \\ 8978 & -35.8 & 32.9 & 323.7 & 0 \\ 8964 & -36.2 & 32.7 & 323.9 & 0 \\ 9067 & -36.5 & 32.8 & 319.4 & 0 \\ 9149 & -36.8 & 32.8 & 319.6 & 0 \\ 9258 & -37.1 & 33.0 & 315.1 & 0 \\ 9114 & -37.5 & 33.5 & 312.1 & 0 \\ 9238 & -38.2 & 34.1 & 308.6 & 0 \\ 9343 & -38.7 & 34.6 & 307.7 & 0 \\ 9430 & -38.8 & 35.0 & 307.8 & 0 \\ 9534 & -39.8 & 36.2 & 302.0 & 0 \\ 9487 & -40.6 & 37.0 & 297.5 & 0\end{array}$

$-21.9$

29.4

0

0

0

0

0

0

0

0

0

0

0

0

0

0

0

0

0

0

0

0

0

0

0

0

0

0

0

0

0

0

0

0

0 temp rel hum pressure 5-13um 13-26um 26-50um mb counts/1 counts/1 counts/1 counts/1

$\operatorname{deg} C$
0
0
0
0
0
0
0
0
0
0
0
0
0
0
0
0
0
0
0
0
0
0
0
0
0
0
0
0
0
0
0
0
0
0
0
0
0
0
0
0
0
0

$\begin{array}{ll}0 & 0 \\ 0 & 0 \\ 0 & 0 \\ 0 & 0 \\ 0 & 0 \\ 0 & 0 \\ 0 & 0 \\ 0 & 0 \\ 0 & 0 \\ 0 & 0 \\ 0 & 0 \\ 0 & 0 \\ 0 & 0 \\ 0 & 0 \\ 0 & 0 \\ 0 & 0 \\ 0 & 0 \\ 0 & 0 \\ 0 & 0 \\ 0 & 0 \\ 0 & 0 \\ 0 & 0 \\ 0 & 0 \\ 0 & 0 \\ 0 & 0 \\ 0 & 0 \\ 0 & 0 \\ 0 & 0 \\ 0 & 0 \\ 0 & 0 \\ 0 & 0 \\ 0 & 0 \\ 0 & 0 \\ 0 & 0 \\ 0 & 0 \\ 0 & 0 \\ 0 & 0 \\ 0 & 0 \\ 0 & 0 \\ 0 & 0 \\ 0 & 0 \\ 0 & 0\end{array}$




\section{APR8 8}

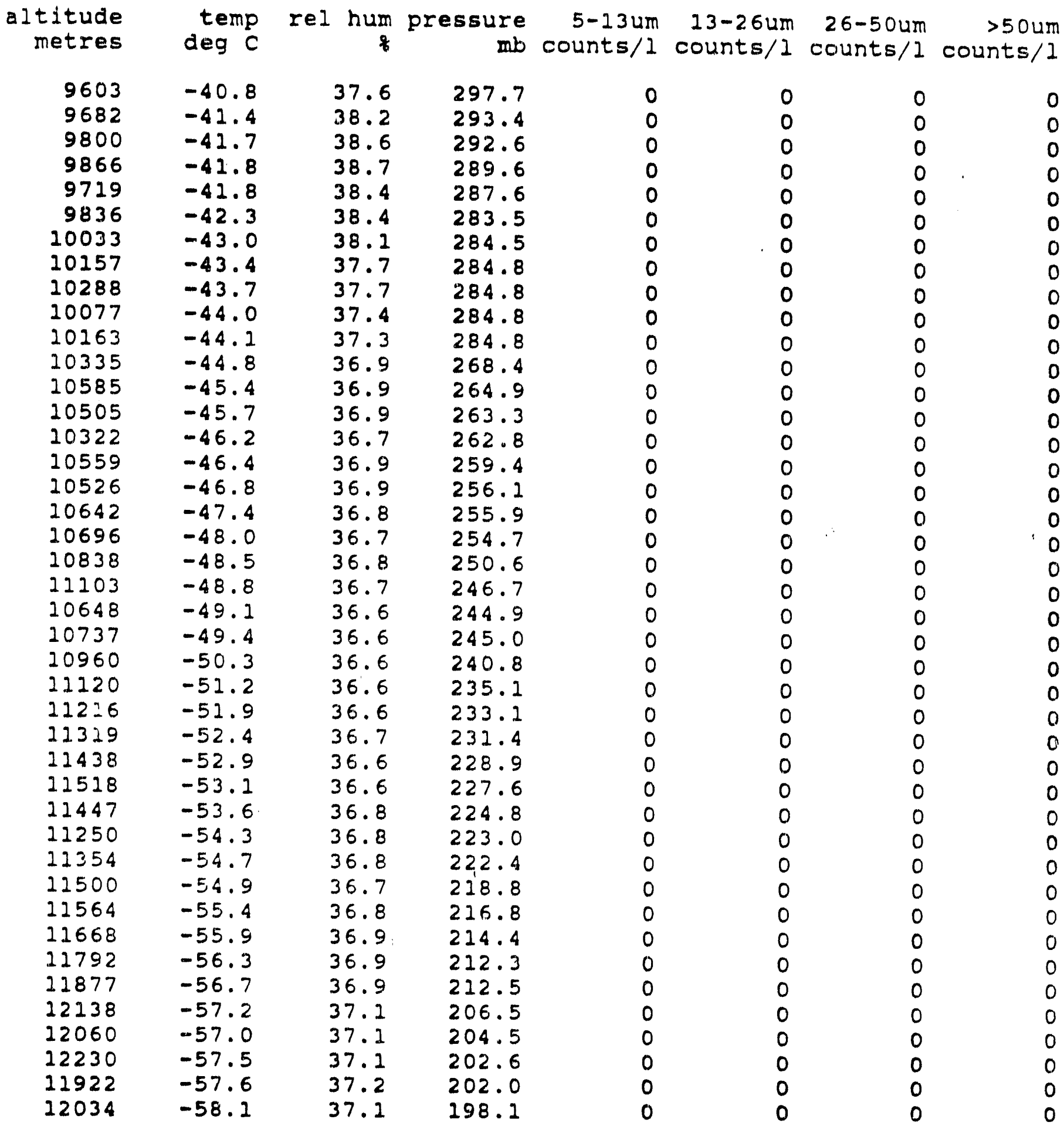




\section{APR8 8}

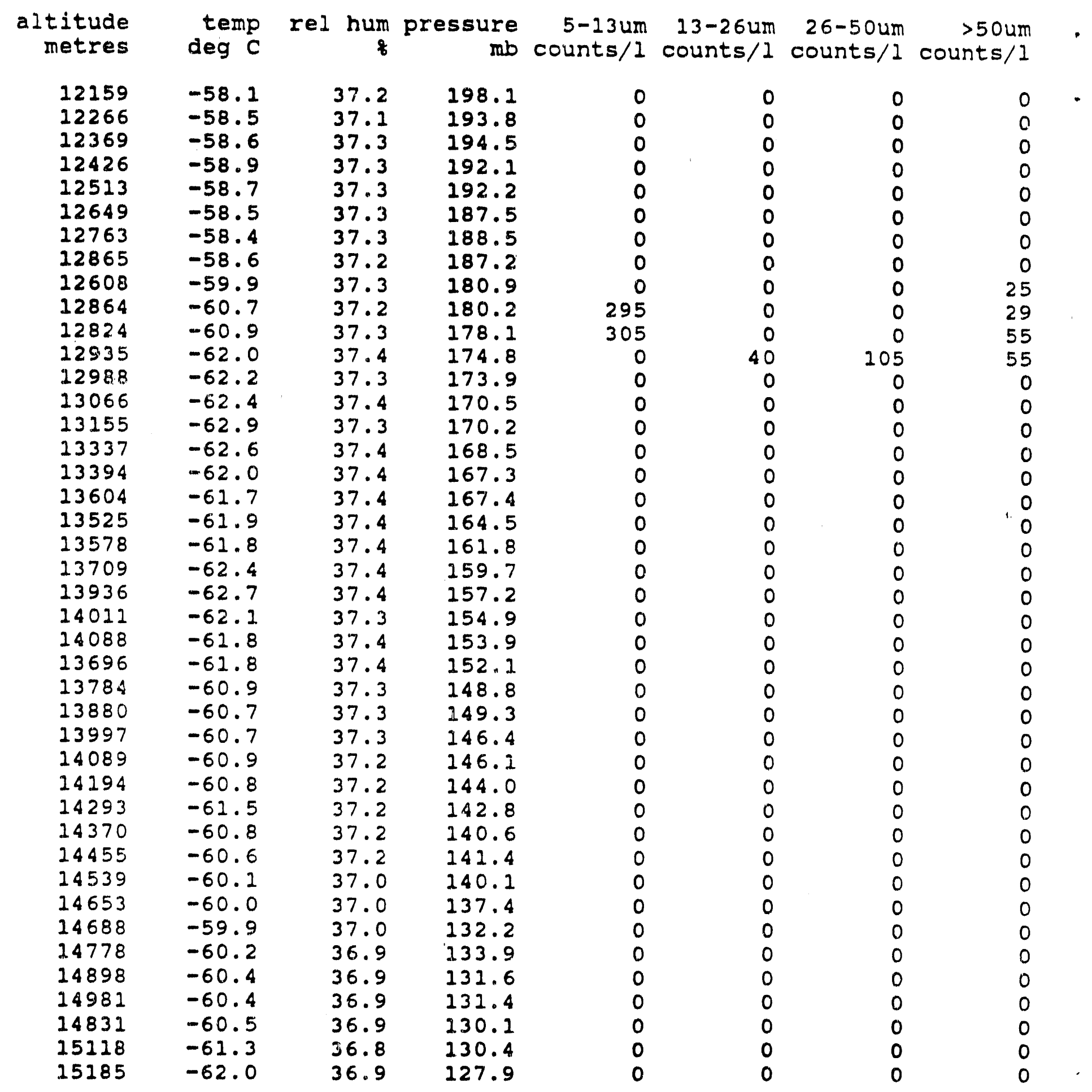




\section{APRB 8}

altitude metres

15561

15371

15405

15110

15587

15427

15518

15610

15701

15793

15884

15976

16067

16113

16120

16128

16159

16279

16193

16447

16737

16516

16870

16638

16992

16790

17104

17157

16842

17206

10000

17086

16759

17563

17018

17684

17715

17713

17574

17839

17896

17933 temp rel hum pressure 5-13um 13-26um 26-50um >50um $\operatorname{deg} c$

if mb counts $/ 1$ counts $/ 1$ counts $/ 1$ counts $/ 1$

$-62.7$

36.8

124.6

125.3

122.7

36.8

$\begin{array}{ll}37.0 & 121.4 \\ 36.9 & 120.2\end{array}$

$36.9 \quad 118.0$

$37.0 \quad 121.2$

$36.8 \quad 117.5$

$37.0 \quad 114.7$

$38.0 \quad 112.2$

$35.8 \quad 112.5$

$36.7 \quad 113.0$

$36.8 \quad 111.2$

$36.8 \quad 110.2$

$36.8 \quad 108.7$

$36.8 \quad 107.8$

$36.7 \quad 107.4$

$36.7 \quad 102.9$

36.5

36.7

103.1

99.4

99.7

99.8

97.4

97.9

97.8

95.1

91.5

93.8

90.2

92.6

89.2

89.2

88.7

89.7

87.3

85.5

86.9

83.1

84.3

81.0

82.3

81.0

0
0
0
0
0
0
0
0
0
0
0
0
0
0
0
0
0
0
0
0
0
0
0
0
0
0
0
0
0
0
0
0
0
0
0
0
0
0
0
0
0
0
0

36.6

36.7

36.7
$-66.1$

$-66.8$

$\begin{array}{ll}0 & 0 \\ 0 & 0 \\ 0 & 0 \\ 0 & 0 \\ 0 & 0 \\ 0 & 0 \\ 0 & 0 \\ 0 & 0 \\ 0 & 0 \\ 0 & 0 \\ 0 & 0 \\ 0 & 0 \\ 0 & 0 \\ 0 & 0 \\ 0 & 0 \\ 0 & 0 \\ 0 & 0 \\ 0 & 0 \\ 0 & 0 \\ 0 & 0 \\ 0 & 0 \\ 0 & 0 \\ 0 & 0 \\ 0 & 0 \\ 0 & 0 \\ 0 & 0 \\ 0 & 0 \\ 0 & 0 \\ 0 & 0 \\ 0 & 0 \\ 0 & 0 \\ 0 & 0 \\ 0 & 0 \\ 0 & 0 \\ 0 & 0 \\ 0 & 0 \\ 0 & 0 \\ 0 & 0 \\ 0 & 0 \\ 0 & 0 \\ 0 & 0 \\ 0 & 0 \\ 0 & 0\end{array}$


15APR 8

\begin{tabular}{|c|c|c|c|c|c|c|c|}
\hline $\begin{array}{r}\text { altitude } \\
\text { metres }\end{array}$ & $\begin{array}{l}\text { temp } \\
\text { deg } C\end{array}$ & rel hum & $\begin{array}{r}\text { pressure } \\
\text { mb }\end{array}$ & $\begin{array}{r}5-13 u m \\
\text { counts } / 1\end{array}$ & $\begin{array}{c}13-26 u m \\
\text { counts } / 1\end{array}$ & $\begin{array}{l}26-50 u m \\
\text { counts } / 1\end{array}$ & $\begin{array}{r}>50 \text { um } \\
\text { counts } / 1\end{array}$ \\
\hline $\begin{array}{l}18002 \\
18115 \\
18161 \\
18218 \\
18288 \\
18344 \\
18373 \\
18438 \\
18495 \\
18524 \\
18648 \\
18683 \\
18716\end{array}$ & $\begin{array}{l}-66.4 \\
-65.1 \\
-65.0 \\
-64.7 \\
-65.0 \\
-65.0 \\
-64.7 \\
-64.4 \\
-64.1 \\
-64.1 \\
-64.4 \\
-64.6 \\
-64.3\end{array}$ & $\begin{array}{l}36.8 \\
36.7 \\
36.8 \\
36.7 \\
36.8 \\
36.6 \\
36.7 \\
36.6 \\
36.6 \\
36.7 \\
36.6 \\
36.6 \\
36.7\end{array}$ & $\begin{array}{l}81.6 \\
80.1 \\
78.8 \\
76.5 \\
77.6 \\
75.8 \\
74.3 \\
73.5 \\
73.6 \\
75.0 \\
72.2 \\
73.4 \\
71.3\end{array}$ & $\begin{array}{l}0 \\
0 \\
0 \\
0 \\
0 \\
0 \\
0 \\
0 \\
0 \\
0 \\
0 \\
0 \\
0\end{array}$ & $\begin{array}{l}0 \\
0 \\
0 \\
0 \\
0 \\
0 \\
0 \\
0 \\
0 \\
0 \\
0 \\
0 \\
0\end{array}$ & $\begin{array}{l}0 \\
0 \\
0 \\
0 \\
0 \\
0 \\
0 \\
0 \\
0 \\
0 \\
0 \\
0 \\
0\end{array}$ & $\begin{array}{l}0 \\
0 \\
0 \\
0 \\
0 \\
0 \\
0 \\
0 \\
0 \\
0 \\
0 \\
0 \\
0\end{array}$ \\
\hline
\end{tabular}




\section{JUN88}

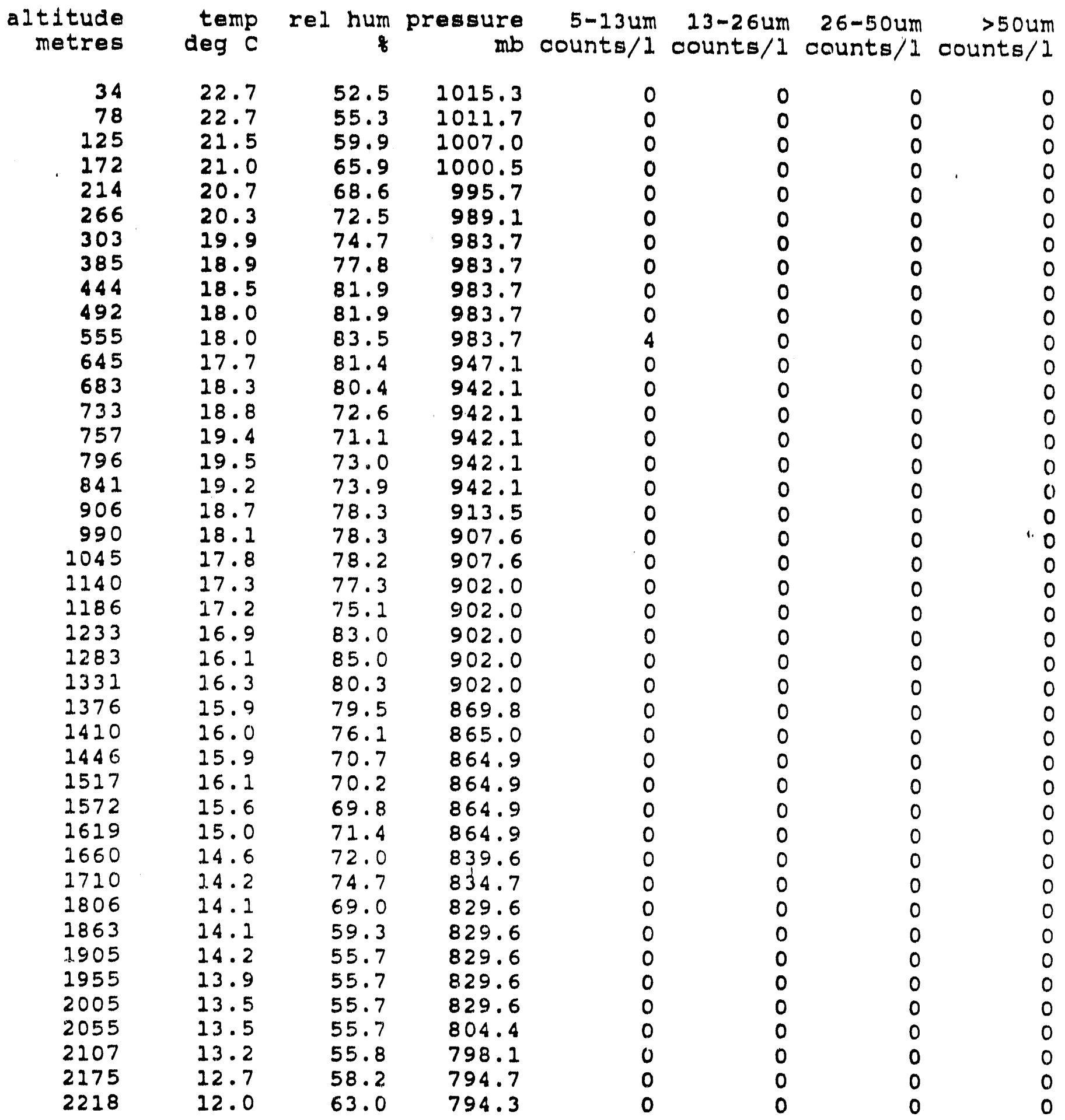




\section{JUN8 8}

\begin{tabular}{|c|c|c|c|c|c|c|c|}
\hline $\begin{array}{l}\text { altitude } \\
\text { metres }\end{array}$ & $\begin{array}{l}\text { temp } \\
\operatorname{deg} c\end{array}$ & rel hum & $\begin{array}{r}\text { pressure } \\
\text { mb }\end{array}$ & $\begin{array}{c}5-13 u m \\
\text { counts } / 1\end{array}$ & $\begin{array}{c}13-26 \mathrm{um} \\
\text { counts/1 }\end{array}$ & $\begin{array}{c}26-50 u m \\
\text { counts } / 1\end{array}$ & $\begin{array}{r}>50 u m \\
\text { counts } / 1\end{array}$ \\
\hline $\begin{array}{l}2270 \\
2307 \\
2362 \\
2450 \\
2545 \\
2583 \\
2633 \\
2670 \\
2720 \\
2762 \\
2814 \\
2865 \\
2918 \\
2962 \\
3010 \\
3067 \\
3119 \\
3303 \\
3341 \\
3392 \\
3425 \\
3474 \\
3519 \\
3557 \\
3608 \\
3654 \\
3698 \\
3744 \\
3792 \\
3945 \\
3999 \\
4039 \\
4080 \\
4134 \\
4179 \\
4224 \\
4273 \\
4313 \\
4369 \\
4407 \\
4453 \\
4499\end{array}$ & $\begin{array}{l}11.8 \\
11.3 \\
11.0 \\
10.5 \\
10.1 \\
9.7 \\
9.7 \\
9.5 \\
9.0 \\
8.7 \\
8.3 \\
7.9 \\
7.4 \\
6.9 \\
6.4 \\
6.4 \\
6.2 \\
5.0 \\
4.7 \\
4.8 \\
4.7 \\
4.7 \\
4.3 \\
4.2 \\
4.0 \\
4.0 \\
3.5 \\
3.1 \\
2.8 \\
3.6 \\
3.7 \\
3.5 \\
3.2 \\
2.9 \\
2.3 \\
2.3 \\
1.8 \\
1.6 \\
1.0 \\
0.7 \\
0.1 \\
0.1\end{array}$ & $\begin{array}{l}64.8 \\
63.8 \\
65.3 \\
64.4 \\
65.5 \\
66.0 \\
74.7 \\
75.7 \\
76.8 \\
81.5 \\
87.3 \\
88.5 \\
89.5 \\
89.0 \\
90.2 \\
90.2 \\
87.8 \\
92.2 \\
92.3 \\
88.4 \\
81.4 \\
75.4 \\
67.1 \\
62.2 \\
62.2 \\
63.2 \\
66.8 \\
64.9 \\
52.7 \\
24.5 \\
25.7 \\
26.6 \\
28.5 \\
28.5 \\
28.5 \\
28.9 \\
29.2 \\
29.0 \\
30.2 \\
31.2 \\
30.7 \\
29.5\end{array}$ & $\begin{array}{l}794.3 \\
794.3 \\
794.3 \\
763.9 \\
759.0 \\
759.0 \\
759.0 \\
759.0 \\
759.0 \\
737.4 \\
732.7 \\
727.1 \\
727.3 \\
727.3 \\
727.3 \\
727.3 \\
727.3 \\
696.5 \\
696.5 \\
696.5 \\
696.5 \\
696.5 \\
670.8 \\
667.4 \\
667.4 \\
664.0 \\
664.0 \\
664.0 \\
664\end{array}$ & $\begin{array}{l}0 \\
0 \\
0 \\
0 \\
0 \\
0 \\
0 \\
0 \\
0 \\
0 \\
0 \\
0 \\
0 \\
0 \\
0 \\
0 \\
0 \\
0 \\
0 \\
0 \\
0 \\
0 \\
0 \\
0 \\
0 \\
0 \\
0 \\
0 \\
0 \\
0 \\
0 \\
0 \\
0 \\
0 \\
0 \\
0 \\
0 \\
0 \\
0 \\
0 \\
0 \\
0\end{array}$ & $\begin{array}{l}0 \\
0 \\
0 \\
0 \\
0 \\
0 \\
0 \\
0 \\
0 \\
0 \\
0 \\
0 \\
0 \\
0 \\
0 \\
0 \\
0 \\
0 \\
0 \\
0 \\
0 \\
0 \\
0 \\
0 \\
0 \\
0 \\
0 \\
0 \\
0 \\
0 \\
0 \\
0 \\
0 \\
0 \\
0 \\
0 \\
0 \\
0 \\
0 \\
0 \\
0 \\
0\end{array}$ & $\begin{array}{l}0 \\
0 \\
0 \\
0 \\
0 \\
0 \\
0 \\
0 \\
0 \\
0 \\
0 \\
0 \\
0 \\
0 \\
0 \\
0 \\
0 \\
0 \\
0 \\
0 \\
0 \\
0 \\
0 \\
0 \\
0 \\
0 \\
0 \\
0 \\
0 \\
0 \\
0 \\
0 \\
0 \\
0 \\
0 \\
0 \\
0 \\
0 \\
0 \\
0 \\
0\end{array}$ & $\begin{array}{l}0 \\
0 \\
0 \\
0 \\
0 \\
0 \\
0 \\
0 \\
0 \\
0 \\
0 \\
0 \\
0 \\
0 \\
0 \\
0 \\
0 \\
0 \\
0 \\
0 \\
0 \\
0 \\
0 \\
0 \\
0 \\
0 \\
0 \\
0 \\
0 \\
0 \\
0 \\
0 \\
0 \\
0 \\
0 \\
0 \\
0 \\
0 \\
0 \\
0 \\
0 \\
0\end{array}$ \\
\hline
\end{tabular}


10 JUN 88

altitude metres

temp rel hum pressure $\operatorname{deg} \mathrm{C}$ mb counts/1 counts/1 counts/1 counts/1

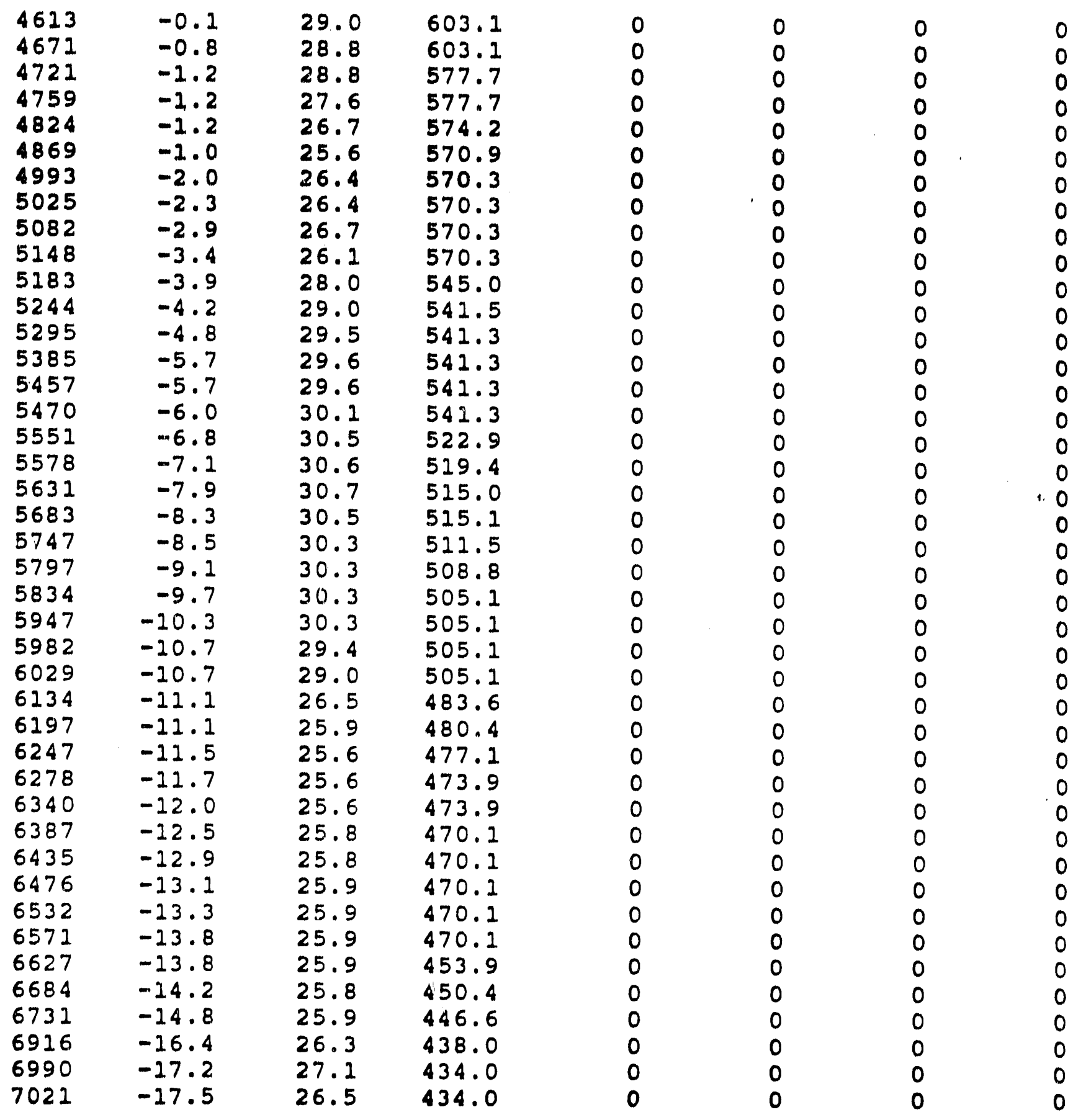




\section{JUN8B}

altitude metres

$\begin{array}{llll}7082 & -18.0 & 26.3 & 434.0 \\ 7139 & -18.7 & 26.5 & 424.5 \\ 7212 & -18.9 & 26.5 & 424.5 \\ 7229 & -18.9 & 26.3 & 424.5 \\ 7309 & -19.5 & 26.3 & 414.8 \\ 7354 & -19.8 & 26.5 & 414.8 \\ 7384 & -20.2 & 26.0 & 411.8 \\ 7457 & -20.7 & 26.3 & 408.5 \\ 7497 & -21.0 & 26.5 & 408.5 \\ 7656 & -22.3 & 26.7 & 408.5 \\ 7718 & -22.7 & 28.1 & 388.5 \\ 7762 & -23.2 & 27.1 & 385.6 \\ 7827 & -23.6 & 27.0 & 383.1 \\ 7881 & -24.3 & 27.4 & 383.0 \\ 7939 & -24.3 & 27.4 & 379.8 \\ 7983 & -24.7 & 27.7 & 376.3 \\ 8070 & -25.1 & 28.1 & 373.8 \\ 8093 & -25.2 & 28.1 & 370.6 \\ 8162 & -25.9 & 29.1 & 368.0 \\ 8204 & -26.5 & 28.1 & 364.9 \\ 8274 & -26.7 & 28.0 & 364.9 \\ 8323 & -27.3 & 27.8 & 362.1 \\ 8413 & -27.8 & 27.9 & 356.4 \\ 8453 & -28.3 & 28.1 & 353.9 \\ 8540 & -29.0 & 28.1 & 350.2 \\ 8555 & -29.4 & 27.8 & 347.4 \\ 8669 & -30.0 & 28.3 & 342.9 \\ 8720 & -30.8 & 28.5 & 339.7 \\ 8772 & -30.9 & 28.7 & 337.0 \\ 8815 & -31.2 & 28.7 & 334.7 \\ 8871 & -31.3 & 28.5 & 332.0 \\ 8911 & -31.2 & 28.5 & 329.7 \\ 8957 & -31.1 & 28.1 & 327.3 \\ 9044 & -31.5 & 28.1 & 324.2 \\ 9081 & -31.4 & 27.9 & 321.7 \\ 9184 & -32.3 & 27.9 & 316.5 \\ 9220 & -32.9 & 27.8 & 316.6 \\ 9285 & -32.9 & 27.8 & 313.5 \\ 9327 & -32.6 & 27.9 & 311.8 \\ 9386 & -33.2 & 28.1 & 309.6 \\ 9407 & -33.4 & 27.5 & 307.8 \\ 9591 & -34.0 & 28.0 & 304.9\end{array}$

temp rel hum pressure mb counts/1 counts/1 counts/1

$>50 u m$

$\begin{array}{ll}0 & 0 \\ 0 & 0 \\ 0 & 0 \\ 0 & 0 \\ 0 & 0 \\ 0 & 0 \\ 0 & 0 \\ 0 & 0 \\ 0 & 0 \\ 0 & 0 \\ 0 & 0 \\ 0 & 0 \\ 0 & 0 \\ 0 & 0 \\ 0 & 0 \\ 0 & 0 \\ 0 & 0 \\ 0 & 0 \\ 0 & 0 \\ 0 & 0 \\ 0 & 0 \\ 0 & 0 \\ 0 & 0 \\ 0 & 0 \\ 0 & 0 \\ 0 & 0 \\ 0 & 0 \\ 0 & 0 \\ 0 & 0 \\ 0 & 0 \\ 0 & 0 \\ 0 & 0 \\ 0 & 0 \\ 0 & 0 \\ 0 & 0 \\ 0 & 0 \\ 0 & 0 \\ 0 & 0 \\ 0 & 0 \\ 0 & 0 \\ 0 & 0 \\ 0 & 0 \\ 0 & 0\end{array}$

$\begin{array}{ll}0 & 0 \\ 0 & 0 \\ 0 & 0 \\ 0 & 0 \\ 0 & 0 \\ 0 & 0 \\ 0 & 0 \\ 0 & 0 \\ 0 & 0 \\ 0 & 0 \\ 0 & 0 \\ 0 & 0 \\ 0 & 0 \\ 0 & 0 \\ 0 & 0 \\ 0 & 0 \\ 0 & 0 \\ 0 & 0 \\ 0 & 0 \\ 0 & 0 \\ 0 & 0 \\ 0 & 0 \\ 0 & 0 \\ 0 & 0 \\ 0 & 0 \\ 0 & 0 \\ 0 & 0 \\ 0 & 0 \\ 0 & 0 \\ 0 & 0 \\ 0 & 0 \\ 0 & 0 \\ 0 & 0 \\ 0 & 0 \\ 0 & 0 \\ 0 & 0 \\ 0 & 0 \\ 0 & 0 \\ 0 & 0 \\ 0 & 0 \\ 0 & 0 \\ 0 & 0\end{array}$


IOJUNB8

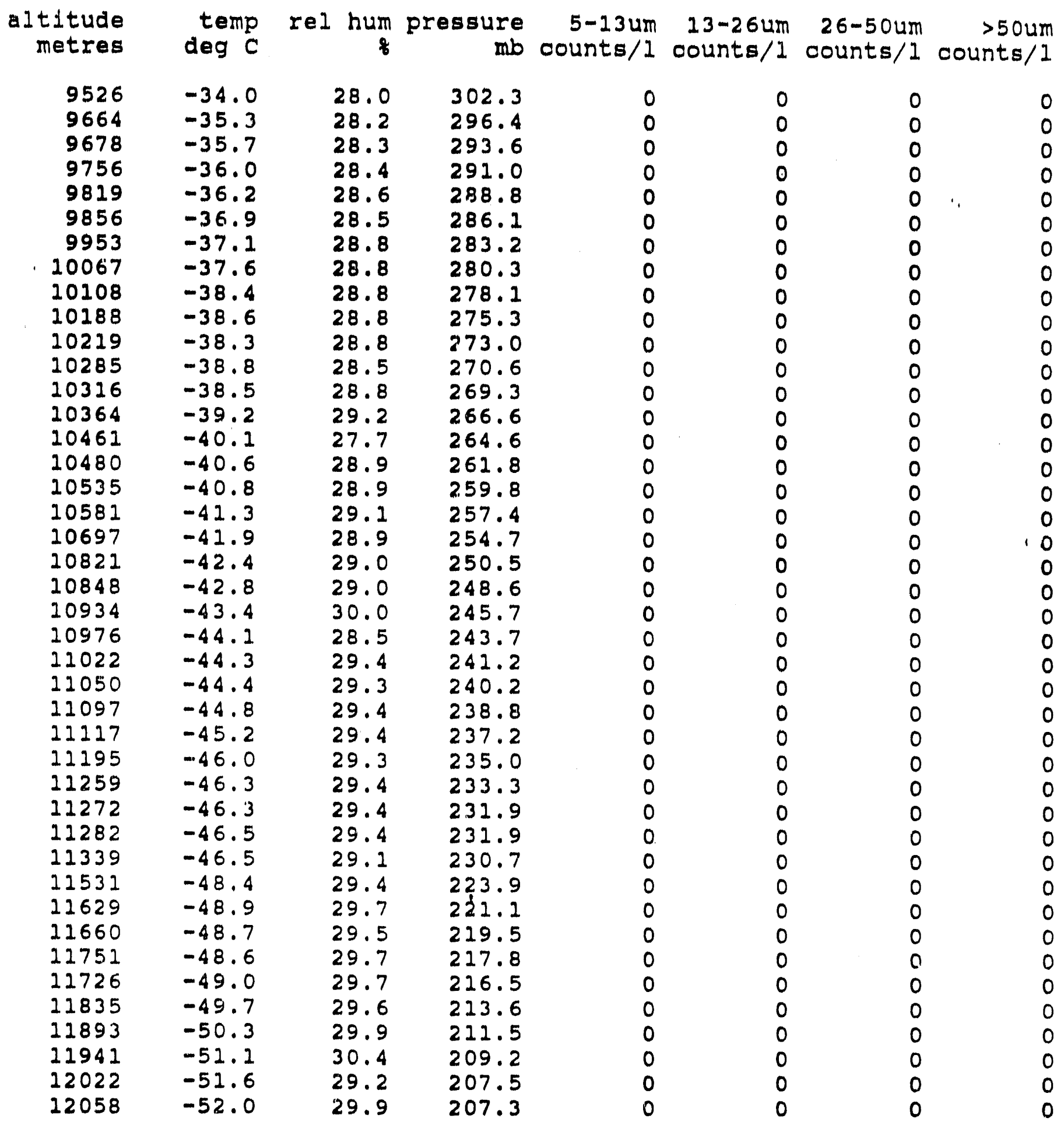




\section{JUN88}

altitude metres

temp rel hum pressure mb counts/I counts/1 counts/1

$>50$ um deq $C$

is pressure

5-13um $13-26 u m$
counts $/ 1$ counts/1

$\begin{array}{llll}12132 & -52.0 & 30.1 & 205.3 \\ 12204 & -52.7 & 30.3 & 203.0 \\ 12274 & -53.0 & 30.4 & 201.2 \\ 12397 & -54.6 & 30.6 & 195.4 \\ 12405 & -54.7 & 30.8 & 193.3 \\ 12517 & -55.1 & 30.6 & 192.1 \\ 12567 & -56.0 & 30.9 & 190.0 \\ 12652 & -56.4 & 30.5 & 188.0 \\ 12715 & -56.7 & 31.0 & 185.5 \\ 12728 & -57.0 & 31.1 & 183.5 \\ 12853 & -57.6 & 31.2 & 181.5 \\ 12885 & -58.0 & 31.2 & 181.4 \\ 12971 & -58.0 & 31.2 & 179.3 \\ 13030 & -57.7 & 31.2 & 178.1 \\ 13268 & -59.6 & 31.2 & 176.3 \\ 13150 & -38.9 & 31.3 & 174.2 \\ 13272 & -59.5 & 31.3 & 170.6 \\ 13292 & -59.5 & 32.6 & 168.7 \\ 13379 & -59.7 & 31.2 & 167.1 \\ 13440 & -60.3 & 31.5 & 165.2 \\ 13600 & -61.0 & 31.5 & 161.3 \\ 13600 & -61.4 & 31.5 & 159.6 \\ 13686 & -62.1 & 31.6 & 158.2 \\ 13714 & -62.6 & 31.7 & 158.2 \\ 13810 & -62.6 & 31.7 & 156.9 \\ 13841 & -62.7 & 31.7 & 155.2 \\ 13873 & -62.5 & 31.7 & 153.5 \\ 14031 & -63.2 & 31.9 & 152.0 \\ 13979 & -63.1 & 32.2 & 150.6 \\ 14124 & -63.4 & 31.9 & 147.9 \\ 14109 & -63.9 & 31.9 & 146.7 \\ 14247 & -62.9 & 31.9 & 145.5 \\ 14207 & -61.9 & 31.5 & 144.6 \\ 14232 & -61.2 & 31.6 & 143.2 \\ 14337 & -61.8 & 32.0 & 141.5 \\ 14357 & -62.2 & 31.8 & 139.9 \\ 14479 & -62.5 & 31.6 & 139.9 \\ 14580 & -62.8 & 31.3 & 136.5 \\ 14649 & -63.0 & 32.9 & 134.8 \\ 14715 & -62.7 & 30.8 & 133.6 \\ 14819 & -62.9 & 31.7 & 132.3 \\ 14786 & -62.8 & 31.8 & 131.1 \\ & & & \end{array}$

0
0
0
0
0
0
0
0
0
0
0
0
0
0
0
0
0
0
0
0
0
0
0
0
4
7
4
0
0
0
0
0
0
0
0
0
0
0
0
0
0
0

0
0
0
0
0
0
0
0
0
0
0
0
0
0
0
0
0
0
0
0
0
0
0
0
0
0
0
4
0
0
0
0
0
0
0
0
0
0
0
0
0
0
0




\section{$10 J U N 88$}

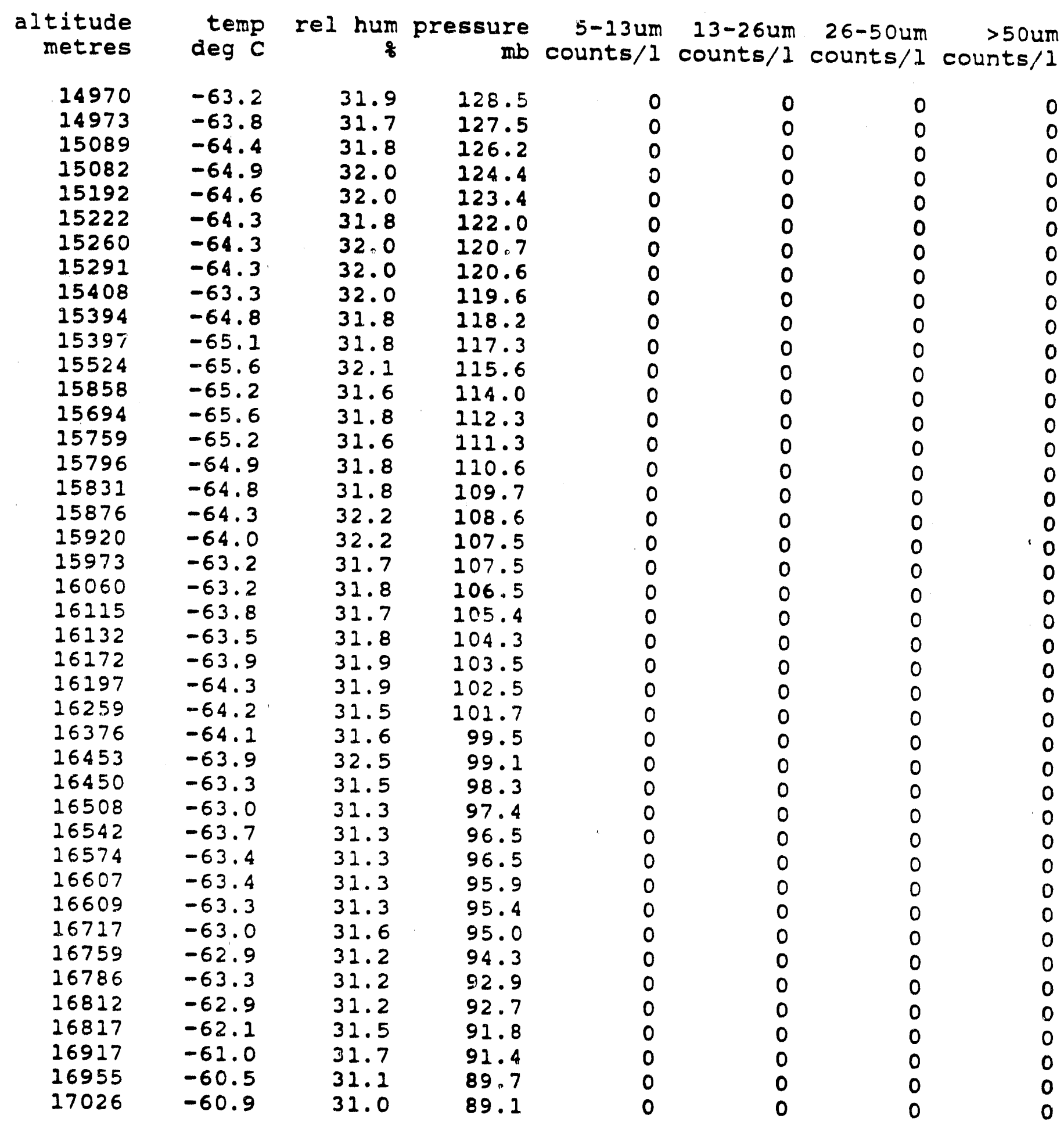




\section{JUN88}

\begin{tabular}{|c|c|c|c|c|c|c|c|}
\hline $\begin{array}{l}\text { Ititude } \\
\text { metres }\end{array}$ & $\begin{array}{l}\text { temp } \\
\operatorname{deg} C\end{array}$ & rel hum & $\begin{array}{r}\text { pressure } \\
\text { mb }\end{array}$ & $\begin{array}{r}5-13 \text { um } \\
\text { counts } / 1\end{array}$ & $\begin{array}{c}13-26 u m \\
\text { counts/1 }\end{array}$ & $\begin{array}{c}26-50 u m \\
\text { counts/I }\end{array}$ & $\begin{array}{r}>50 u m \\
\text { counts } / 1\end{array}$ \\
\hline $\begin{array}{l}17036 \\
17326 \\
17355 \\
17441 \\
17417 \\
17396 \\
17504 \\
17589 \\
17655 \\
17653 \\
17787 \\
17758 \\
17814 \\
17858 \\
17946 \\
17915 \\
17987 \\
18029 \\
18017 \\
18110 \\
18165 \\
18223 \\
18193 \\
18265 \\
18331 \\
18373 \\
18442 \\
18461 \\
18504 \\
18 \\
18546 \\
18625 \\
18648 \\
18661 \\
18710 \\
18749\end{array}$ & $\begin{array}{l}-60.9 \\
-60.9 \\
-61.6 \\
-61.5 \\
-61.3 \\
-62.1 \\
-62.0 \\
-62.8 \\
-62.8 \\
-62.5 \\
-62.9 \\
-62.9 \\
-62.8 \\
-62.3 \\
-61.8 \\
-62.0 \\
-61.1 \\
-61.4 \\
-61.0 \\
-60.2 \\
-59.8 \\
-59.0 \\
-58.5 \\
-57.7 \\
-57.0 \\
-57.0 \\
-56.9 \\
-57.4 \\
-56.5 \\
-56.6 \\
-56.8 \\
-56.3 \\
-55.5 \\
-55.3 \\
-54.8\end{array}$ & $\begin{array}{l}31.0 \\
31.0 \\
31.0 \\
30.8 \\
30.9 \\
31.0 \\
30.8 \\
30.8 \\
30.9 \\
30.9 \\
31.0 \\
30.8 \\
31.6 \\
30.6 \\
30.8 \\
31.2 \\
30.6 \\
30.7 \\
30.6 \\
30.7 \\
30.7 \\
31.7 \\
30.0 \\
30.7 \\
30.5 \\
30.5 \\
30.5 \\
30.4 \\
30.4 \\
30.2 \\
30.2 \\
31.0 \\
30.0 \\
30.2 \\
30.2\end{array}$ & $\begin{array}{l}88.5 \\
87.7 \\
84.1 \\
83.6 \\
82.7 \\
81.9 \\
81.0 \\
80.3 \\
79.7 \\
79.4 \\
78.1 \\
78.1 \\
77.9 \\
77.0 \\
76.5 \\
75.9 \\
75.4 \\
74.7 \\
74.3 \\
73.0 \\
72.5 \\
72.0 \\
71.2 \\
71.0 \\
71.0 \\
70.5 \\
69.7 \\
68.7 \\
68.3 \\
67.6 \\
67.3 \\
66.6 \\
66.1 \\
66.0 \\
66.0\end{array}$ & $\begin{array}{l}0 \\
0 \\
0 \\
0 \\
0 \\
0 \\
0 \\
0 \\
0 \\
0 \\
0 \\
0 \\
0 \\
0 \\
0 \\
0 \\
0 \\
0 \\
0 \\
0 \\
0 \\
0 \\
0 \\
0 \\
0 \\
0 \\
0 \\
0 \\
0 \\
0 \\
0 \\
0 \\
0 \\
0 \\
0\end{array}$ & $\begin{array}{l}0 \\
0 \\
0 \\
0 \\
0 \\
0 \\
0 \\
0 \\
0 \\
0 \\
0 \\
0 \\
0 \\
0 \\
0 \\
0 \\
0 \\
0 \\
0 \\
0 \\
0 \\
0 \\
0 \\
0 \\
0 \\
0 \\
0 \\
0 \\
0 \\
0 \\
0 \\
0 \\
0 \\
0 \\
0\end{array}$ & $\begin{array}{l}0 \\
0 \\
0 \\
0 \\
0 \\
0 \\
0 \\
0 \\
0 \\
0 \\
0 \\
0 \\
0 \\
0 \\
0 \\
0 \\
0 \\
0 \\
0 \\
0 \\
0 \\
0 \\
0 \\
0 \\
0 \\
0 \\
0 \\
0 \\
0 \\
0 \\
0 \\
0 \\
0 \\
0 \\
0\end{array}$ & $\begin{array}{l}0 \\
0 \\
0 \\
0 \\
0 \\
0 \\
0 \\
0 \\
0 \\
0 \\
0 \\
0 \\
0 \\
0 \\
0 \\
0 \\
0 \\
0 \\
0 \\
0 \\
0 \\
0 \\
0 \\
0 \\
0 \\
0 \\
0 \\
0 \\
0 \\
0 \\
0 \\
0 \\
0 \\
0 \\
0\end{array}$ \\
\hline
\end{tabular}




\section{JUN 88}

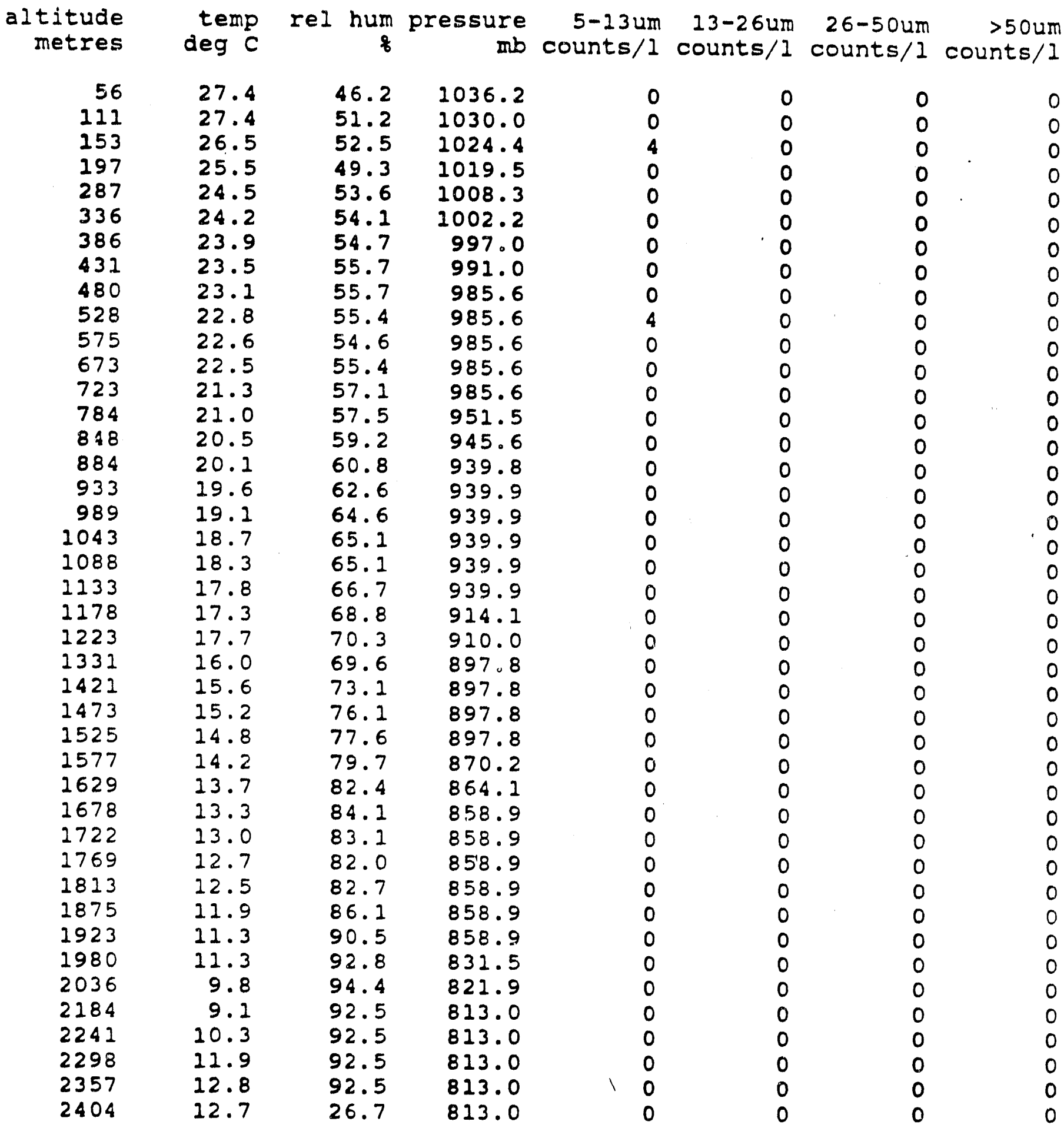




\section{JUN8 8}

\begin{tabular}{|c|c|c|c|c|c|c|c|}
\hline $\begin{array}{l}\text { altitude } \\
\text { metres }\end{array}$ & $\begin{array}{l}\text { temp } \\
\operatorname{deg} C\end{array}$ & rel hum & $\begin{array}{r}\text { pressure } \\
\text { mb }\end{array}$ & $\begin{array}{c}5-13 \mathrm{um} \\
\text { counts/1 }\end{array}$ & $\begin{array}{c}13-26 u m \\
\text { counts } / 1\end{array}$ & $\begin{array}{l}26-50 u m \\
\text { counts/1 }\end{array}$ & $\begin{array}{r}>50 u \pi \\
\text { counts } / 2\end{array}$ \\
\hline 2454 & 12.5 & 25.7 & 787.4 & 0 & 0 & 0 & 0 \\
\hline 2504 & 12.1 & 25.3 & 782.8 & 0 & 0 & 0 & 0 \\
\hline 2535 & 11.8 & 25.9 & 777.8 & 0 & 0 & 0 & 0 \\
\hline 2583 & 11.7 & 25.6 & 774.4 & 0 & 0 & 0 & 0 \\
\hline 2637 & 11.5 & 24.6 & 774.4 & 0 & 0 & 0 & 0 \\
\hline 2683 & 11.3 & 24.3 & 774.4 & 0 & 0 & 0 & 0 \\
\hline 2736 & 11.0 & 24.1 & 774.4 & 0 & 0 & 0 & 0 \\
\hline 2786 & 10.6 & 23.8 & 774.4 & 0 & 0 & 0 & 0 \\
\hline 2927 & 9.4 & 23.4 & 742.2 & 0 & 0 & 0 & 0 \\
\hline 2972 & 9.2 & 23.5 & 737.1 & 0 & 0 & 0 & 0 \\
\hline 3014 & 9.1 & 24.7 & 737.1 & 0 & 0 & 0 & 0 \\
\hline 3072 & 9.5 & 22.1 & 733.9 & 0 & 0 & 0 & 0 \\
\hline 3113 & 9.5 & 23.7 & 733.9 & 0 & 0 & 0 & 0 \\
\hline 3163 & 9.4 & 23.2 & 733.9 & 0 & 0 & 0 & 0 \\
\hline 3211 & 9.5 & 21.3 & 733.9 & 0 & 0 & 0 & 0 \\
\hline 3263 & 9.5 & 21.4 & 733.9 & 0 & 0 & 0 & 0 \\
\hline 3311 & 9.1 & 21.6 & 707.2 & 0 & 0 & 0 & 0 \\
\hline 3364 & 8.6 & 21.8 & 702.6 & 0 & 0 & 0 & .0 \\
\hline 3410 & 8.2 & 21.9 & 698.0 & 0 & 0 & 0 & 0 \\
\hline 3472 & 7.8 & 21.9 & 698.1 & 0 & 0 & 0 & 0 \\
\hline 3525 & 7.4 & 22.1 & 698.1 & 0 & 0 & 0 & 0 \\
\hline 3621 & 7.1 & 22.7 & 698.1 & 0 & 0 & 0 & 0 \\
\hline 3688 & 6.9 & 22.1 & 698.1 & 0 & 0 & 0 & 0 \\
\hline 3727 & 6.7 & 21.9 & 676.1 & 0 & 0 & 0 & 0 \\
\hline 3790 & 6.5 & 20.6 & 672.8 & 0 & 0 & 0 & 0 \\
\hline 3893 & 5.8 & 22.1 & 661.8 & 0 & 0 & 0 & 0 \\
\hline 3957 & 5.4 & 21.9 & 662.1 & 0 & 0 & 0 & 0 \\
\hline 3995 & 5.0 & 21.7 & 658.1 & 0 & 0 & 0 & 0 \\
\hline 4055 & 4.6 & 21.2 & 658.1 & 0 & 0 & 0 & 0 \\
\hline 4100 & 4.3 & 22.4 & 658.1 & 0 & 0 & 0 & 0 \\
\hline 4147 & 4.0 & 21.2 & 658.1 & 0 & 0 & 0 & 0 \\
\hline 4213 & 3.6 & 21.4 & 658.1 & 0 & 0 & 0 & 0 \\
\hline 4254 & 3.1 & 21.9 & 633.2 & 0 & 0 & 0 & 0 \\
\hline 4303 & 2.7 & 22.4 & 629.2 & 0 & 0 & 0 & 0 \\
\hline 4404 & 1.8 & 22.8 & 624.8 & 0 & 0 & 0 & 0 \\
\hline 4455 & 1.3 & 22.8 & 620.8 & 0 & 0 & 0 & 0 \\
\hline 4499 & 0.9 & 23.0 & 620.8 & 0 & 0 & 0 & 0 \\
\hline 4554 & 0.3 & 23.0 & 620.8 & 0 & 0 & 0 & 0 \\
\hline 4605 & 0.0 & 23.0 & 620.8 & 0 & 0 & 0 & 0 \\
\hline 4654 & -0.6 & 22.8 & 620.8 & 0 & 0 & 0 & 0 \\
\hline 4702 & -0.8 & 22.7 & 597.5 & 0 & 0 & 0 & 0 \\
\hline 4770 & -1.3 & 22.7 & 592.5 & 0 & 0 & 0 & 0 \\
\hline
\end{tabular}




\section{JUN 88}

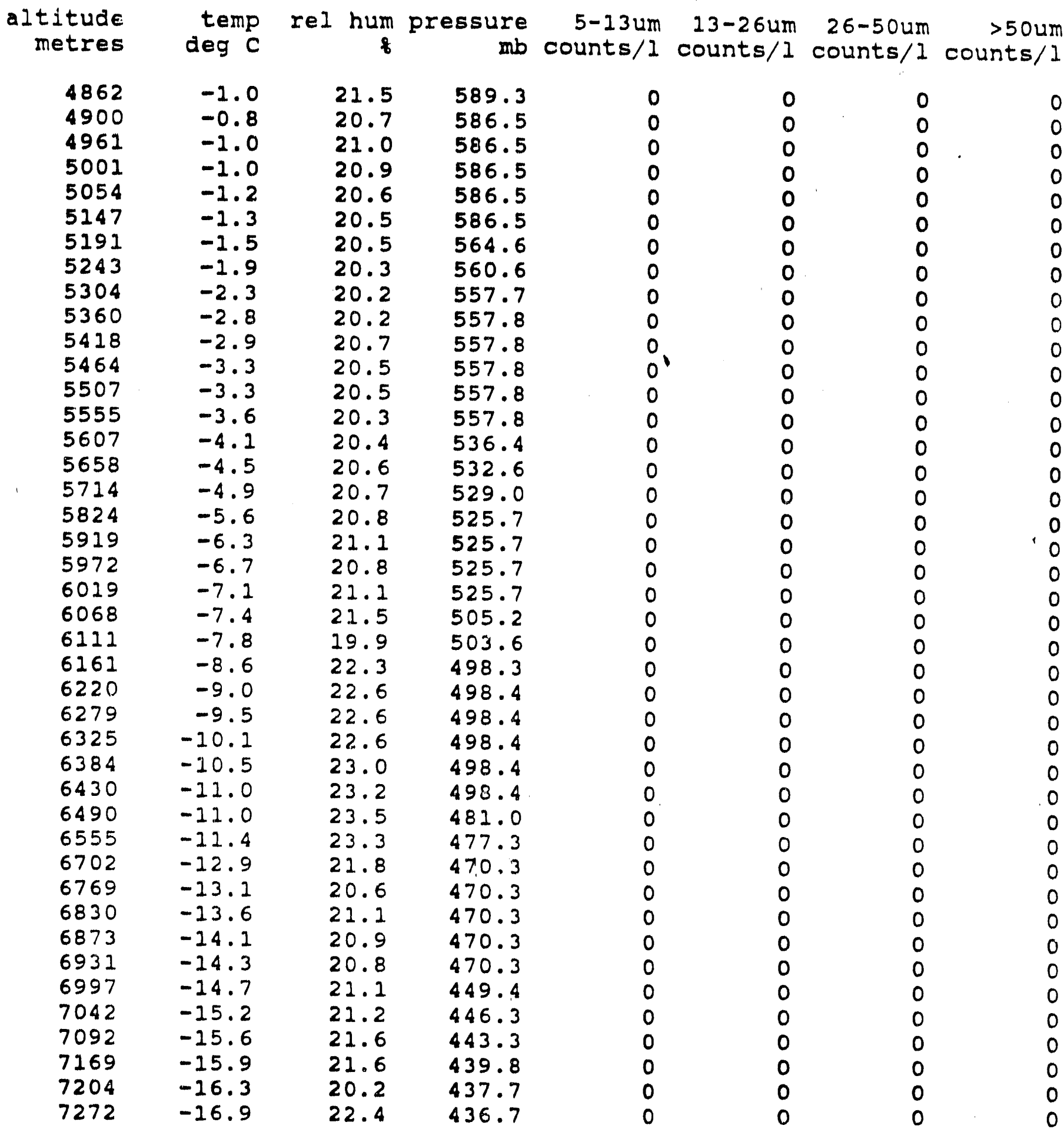




\section{JUN8 8}

\begin{tabular}{|c|c|c|c|}
\hline $\begin{array}{l}\text { altitude } \\
\text { metres }\end{array}$ & $\begin{array}{l}\text { temp } \\
\text { deg } c\end{array}$ & rel hum & $\begin{array}{r}\text { pressure } \\
\text { mb }\end{array}$ \\
\hline $\begin{array}{l}7310 \\
7375 \\
7431 \\
7522 \\
7587 \\
7633 \\
7713 \\
7767 \\
7819 \\
7881 \\
7955 \\
8001 \\
8041 \\
8111 \\
8171 \\
8229 \\
8322 \\
8376 \\
8435 \\
8474 \\
8588 \\
8643 \\
8668 \\
8729 \\
8781 \\
8817 \\
8890 \\
8943 \\
8999 \\
9098 \\
9168 \\
9221 \\
9272 \\
9321 \\
9376 \\
9436 \\
947 \% \\
9580 \\
9640 \\
9702 \\
9747 \\
9802\end{array}$ & $\begin{array}{l}-17.5 \\
-18.0 \\
-18.4 \\
-19.3 \\
-19.7 \\
-20.2 \\
-20.6 \\
-20.8 \\
-21.2 \\
-21.6 \\
-22.1 \\
-22.5 \\
-22.7 \\
-23.3 \\
-24.1 \\
-24 \\
-24.4 \\
-24.8 \\
-24.8 \\
-25.5 \\
-26.0 \\
-26.7 \\
-26 \\
-27.1 \\
-27.4 \\
-27.5 \\
-27.8 \\
-28.3 \\
-28.6 \\
-28.9 \\
-29 \\
-29.5 \\
-30.4 \\
-30.6 \\
-31.0 \\
-31.5 \\
-31.9 \\
-32.3 \\
-32.8 \\
-33.7 \\
-34.3 \\
-35.0 \\
-35.6 \\
-36.1 \\
-36.6\end{array}$ & $\begin{array}{l}22.0 \\
22.2 \\
22.4 \\
23.0 \\
23.3 \\
21.9 \\
22.6 \\
22.5 \\
22.5 \\
22.8 \\
23.1 \\
22.9 \\
22.9 \\
22.4 \\
23.1 \\
23.1 \\
23.4 \\
23.2 \\
23.5 \\
23.4 \\
23.1 \\
23.7 \\
23.5 \\
23.4 \\
23.4 \\
25.6 \\
23.7 \\
23.5 \\
23.8 \\
21.6 \\
23.8 \\
23.8 \\
23.8 \\
23.9 \\
22.8 \\
23.9 \\
24.1 \\
24.2 \\
22.2 \\
24.3 \\
24.6 \\
24.8\end{array}$ & $\begin{array}{l}433.8 \\
433.8 \\
433.8 \\
423.9 \\
415.0 \\
415.0 \\
415.0 \\
408.7 \\
408.7 \\
408.7 \\
398.8 \\
398.8 \\
398.8 \\
389.7 \\
389.7 \\
384.1 \\
384.1 \\
375.7 \\
375.7 \\
375.7 \\
367.2 \\
367.2 \\
361.9 \\
361.9 \\
354.5 \\
354.5 \\
354.5 \\
346.8 \\
346.8 \\
342.4 \\
342.4 \\
342.4 \\
333.0 \\
333.0 \\
328.8 \\
328.8 \\
328.8 \\
320.9 \\
316.9 \\
316.9 \\
316.9 \\
308.3\end{array}$ \\
\hline
\end{tabular}

$13-26 u m$
$26-50 u m$ counts $/ 1$ counts $/ 1$ counts $/ 1$.




\section{JUN 88}

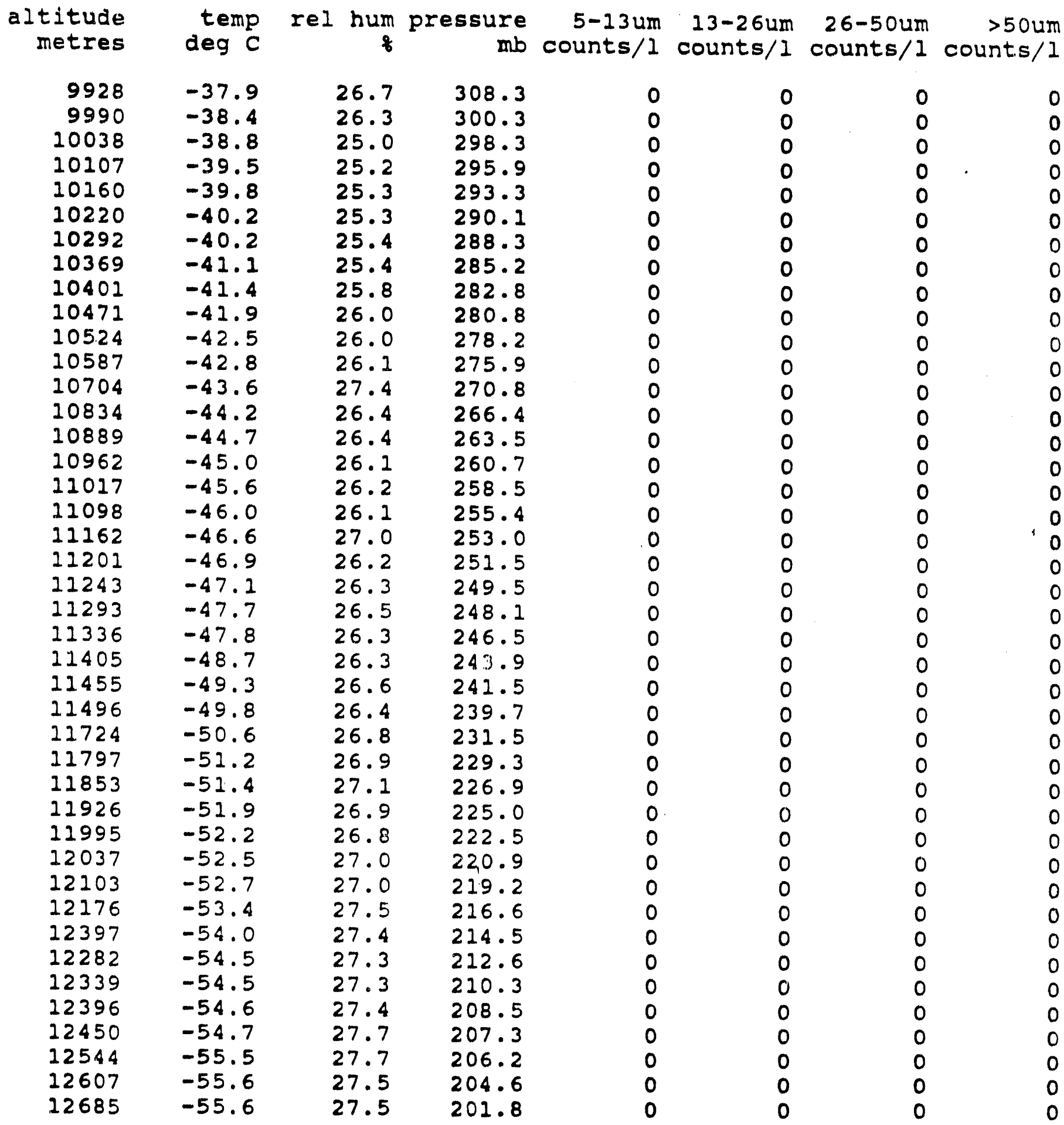


14 JUN8 8

altitude metres

$\begin{array}{llll}12744 & -56.3 & 27.5 & 200.2 \\ 12802 & -56.3 & 27.6 & 198.5 \\ 12848 & -56.5 & 27.8 & 197.1 \\ 12883 & -56.8 & 27.8 & 195.5 \\ 12276 & -57.0 & 28.0 & 193.4 \\ 12122 & -56.7 & 27.9 & 192.6 \\ 12053 & -57.7 & 28.0 & 190.8 \\ 12454 & -58.2 & 28.1 & 188.8 \\ 12904 & -58.4 & 28.0 & 185.5 \\ 13248 & -59.3 & 28.1 & 162.6 \\ 13504 & -59.8 & 28.1 & 178.5 \\ 13547 & -60.5 & 28.2 & 176.6 \\ 13674 & -60.9 & 28.2 & 172.9 \\ 13723 & -61.1 & 28.3 & 171.2 \\ 13807 & -61.2 & 28.4 & 169.8 \\ 13863 & -61.7 & 28.5 & 168.0 \\ 13925 & -62.1 & 28.4 & 165.7 \\ 13982 & -61.8 & 28.5 & 164.7 \\ 14032 & -62.4 & 28.5 & 163.2 \\ 14114 & -63.1 & 28.9 & 160.8 \\ 14178 & -63.2 & 28.8 & 159.3 \\ 14299 & -63.5 & 28.7 & 156.0 \\ 14429 & -63.7 & 29.2 & 154.5 \\ 14433 & -62.8 & 28.6 & 152.8 \\ 14502 & -63.4 & 27.8 & 151.4 \\ 14610 & -63.8 & 28.6 & 149.5 \\ 14679 & -63.7 & 28.2 & 147.8 \\ 14809 & -63.6 & 28.6 & 144.8 \\ 14834 & -63.9 & 29.0 & 146.1 \\ 14890 & -63.8 & 28.5 & 142.0 \\ 14947 & -64.4 & 28.6 & 141.1 \\ 15000 & -64.7 & 28.6 & 140.1 \\ 15062 & -65.2 & 28.6 & 138.6 \\ 15127 & -65.0 & 28.7 & 137.2 \\ 15252 & -64.7 & 28.6 & 135.6 \\ 15304 & -64.7 & 28.7 & 134.7 \\ 15376 & -65.6 & 28.8 & 133.3 \\ 15450 & -66.2 & 28.8 & 131.6 \\ 15568 & -66.7 & 28.7 & 128.9 \\ 15602 & -66.6 & 28.8 & 127.6 \\ 15645 & -66.7 & 28.9 & 126.9 \\ 15703 & -66.8 & 28.9 & 126.3\end{array}$

temp deg $C$ rel hum pressure mb cou

5-13um 13-26um 26-50um counts/1 


\section{$14 \pi U N 88$}

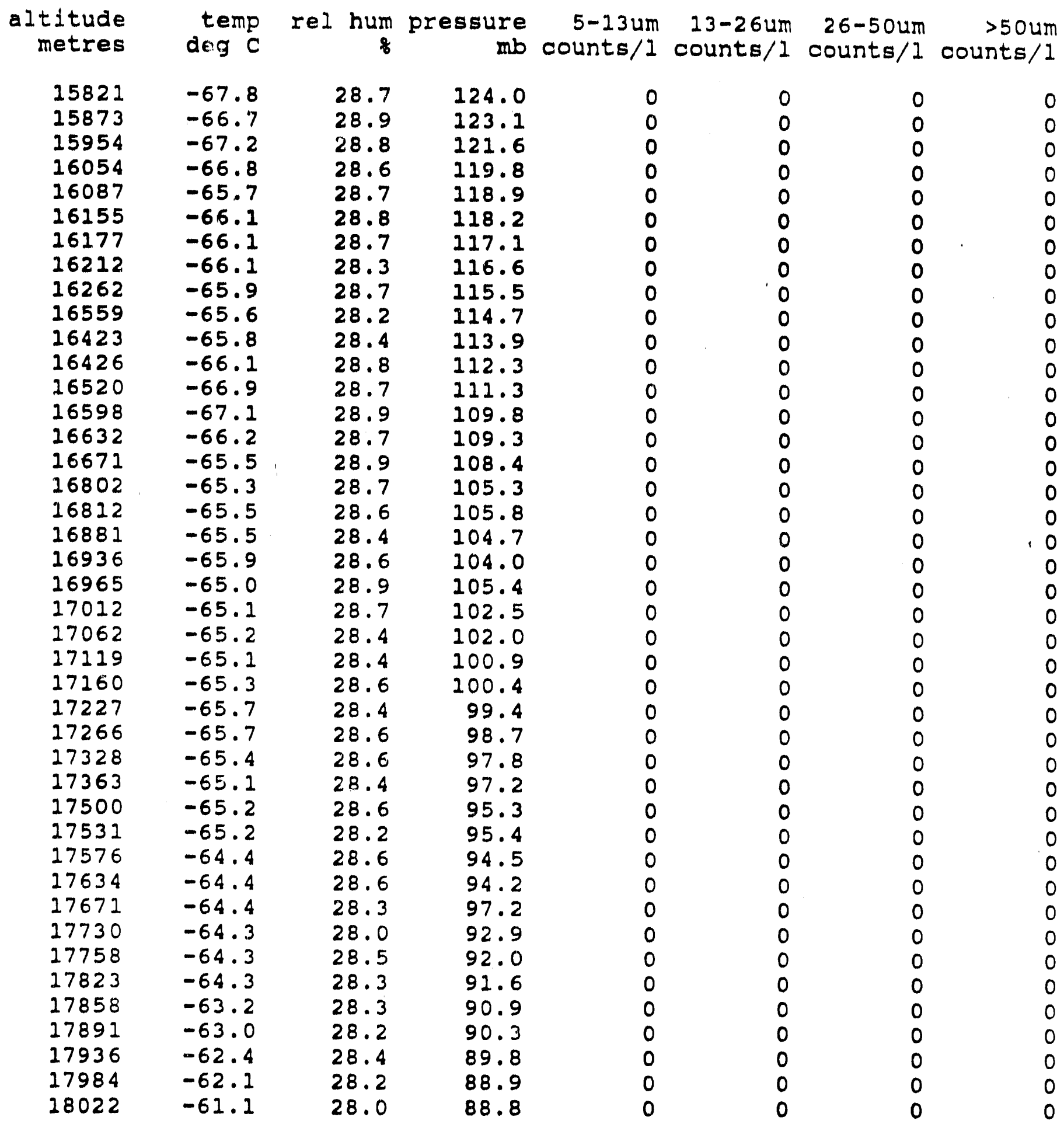




\section{JUN 88}

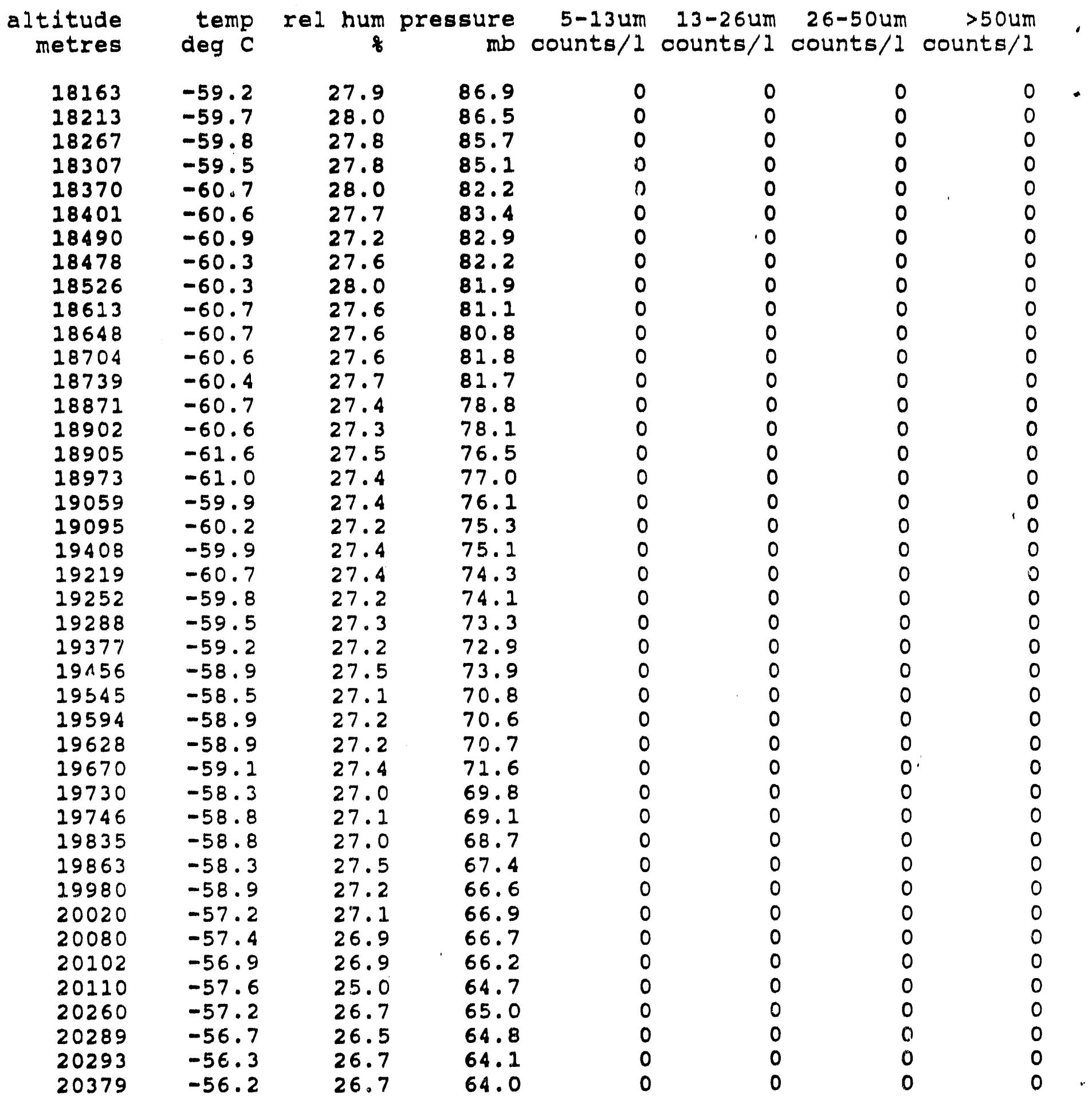




\section{JUN 88}

altitude metres temp rel hum pressure deg $C$

$\begin{array}{llll}20416 & -55.7 & 26.5 & 63.3 \\ 20472 & -55.8 & 26.5 & 62.8 \\ 20522 & -55.7 & 26.6 & 62.5 \\ 20573 & -55.4 & 26.5 & 61.9 \\ 20630 & -55.7 & 26.6 & 61.8 \\ 20686 & -55.8 & 26.5 & 61.1 \\ 20700 & -55.8 & 26.6 & 61.0 \\ 20745 & -55.2 & 26.6 & 62.1 \\ 20861 & -55.2 & 26.2 & 59.8 \\ 20947 & -55.1 & 26.1 & 59.0 \\ 20986 & -54.0 & 26.3 & 58.4 \\ 21061 & -54.5 & 25.9 & 58.1 \\ 21090 & -54.7 & 25.7 & 58.0 \\ 21136 & -54.7 & 26.5 & 57.2 \\ 21168 & -54.7 & 26.1 & 57.2 \\ 21244 & -55.2 & 26.6 & 56.7 \\ 21286 & -54.6 & 26.1 & 56.2 \\ 21322 & -54.4 & 26.2 & 56.2 \\ 21376 & -54.1 & 26.1 & 55.5 \\ 21412 & -54.0 & 26.1 & 55.4 \\ 21466 & -54.0 & 26.1 & 55.0 \\ 21515 & -53.7 & 25.5 & 54.6 \\ 21636 & -53.0 & 25.4 & 53.9 \\ 21693 & -53.0 & 25.6 & 53.3 \\ 21743 & -52.6 & 25.6 & 52.9 \\ 21779 & -52.7 & 25.7 & 53.8 \\ 21831 & -52.3 & 25.5 & 52.0 \\ 21879 & -52.3 & 25.8 & 52.4 \\ 21900 & -51.9 & 25.8 & 51.2 \\ 21945 & -51.7 & 25.6 & 52.1 \\ 22030 & -51.7 & 25.4 & 51.5 \\ 22068 & -51.7 & 25.5 & 51.5 \\ 22139 & -52.0 & 25.5 & 51.0 \\ 22162 & -52.1 & 25.2 & 50.5 \\ 22218 & -52.0 & 25.2 & 50.6 \\ 22369 & -51.4 & 25.3 & 49.6 \\ 22432 & -51.5 & 25.1 & 48.0 \\ 22472 & -51.2 & 25.0 & 48.9 \\ 22529 & -51.2 & 25.2 & 48.7 \\ 22584 & -51.3 & 25.2 & 48.0 \\ 22634 & -51.2 & 25.2 & 49.5 \\ 22691 & -50.9 & 25.1 & 47.6\end{array}$
3

2.8

62.5

61.9

1.1

61.0

2.1

59.0

58.4

58.1

57.2

7.2

56.2

56.2

5.5

5.4

5.0

53.9

53.3

52.9

3.8

2.0

51.2

52.1

51.5

51.5

1.0

50.5

50.6

9.6

8.9

48.7

49.5

47.6 5-13um 13-26um 26-50um >50um mb counts/1 counts/1 counts/1 counts/1

$\begin{array}{ll}0 & 0 \\ 0 & 0 \\ 0 & 0 \\ 0 & 0 \\ 0 & 0 \\ 0 & 0 \\ 0 & 0 \\ 0 & 0 \\ 0 & 0 \\ 0 & 0 \\ 0 & 0 \\ 0 & 0 \\ 0 & 0 \\ 0 & 0 \\ 0 & 0 \\ 0 & 0 \\ 0 & 0 \\ 0 & 0 \\ 0 & 0 \\ 0 & 0 \\ 0 & 0 \\ 0 & 0 \\ 0 & 0 \\ 0 & 0 \\ 0 & 0 \\ 0 & 0 \\ 0 & 0 \\ 0 & 0 \\ 0 & 0 \\ 0 & 0 \\ 0 & 0 \\ 0 & 0 \\ 0 & 0 \\ 0 & 0 \\ 0 & 0 \\ 0 & 0 \\ 0 & 0 \\ 0 & 0 \\ 0 & 0 \\ 0 & 0 \\ 0 & 0 \\ 0 & 0\end{array}$

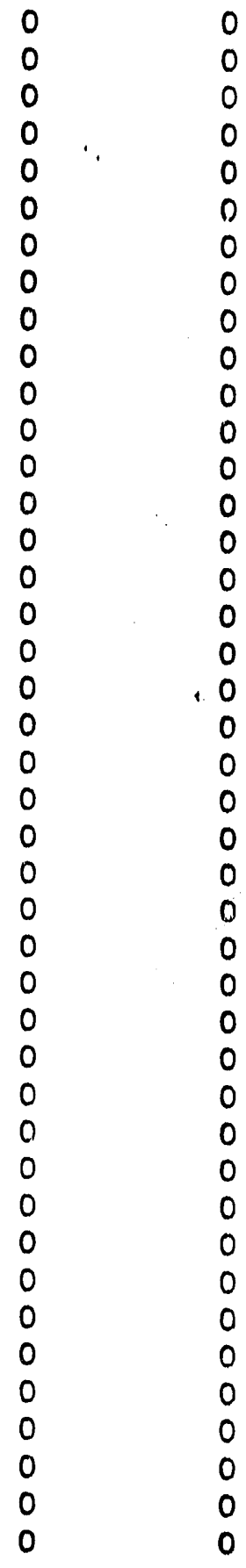




\section{JUN88}

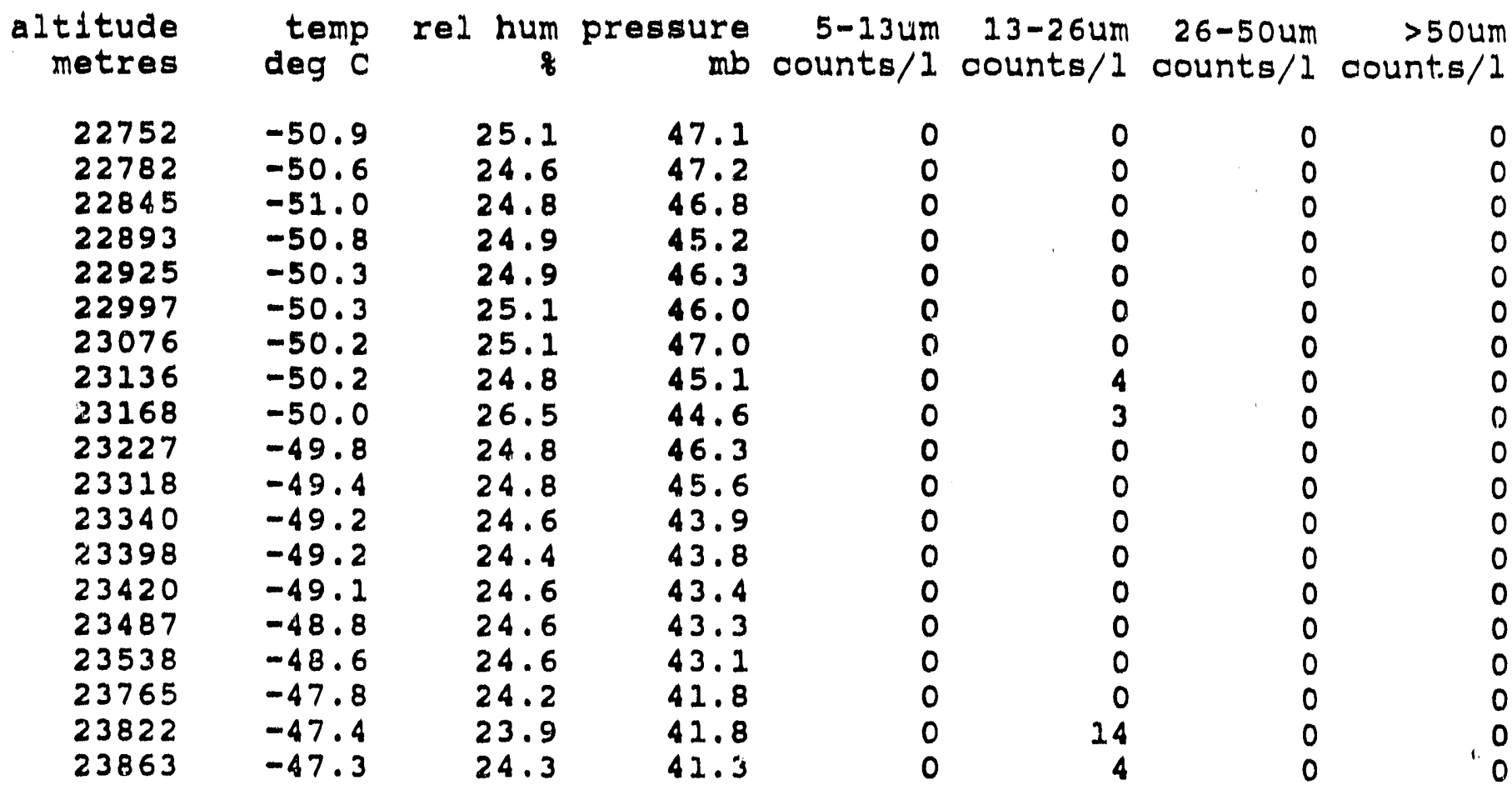




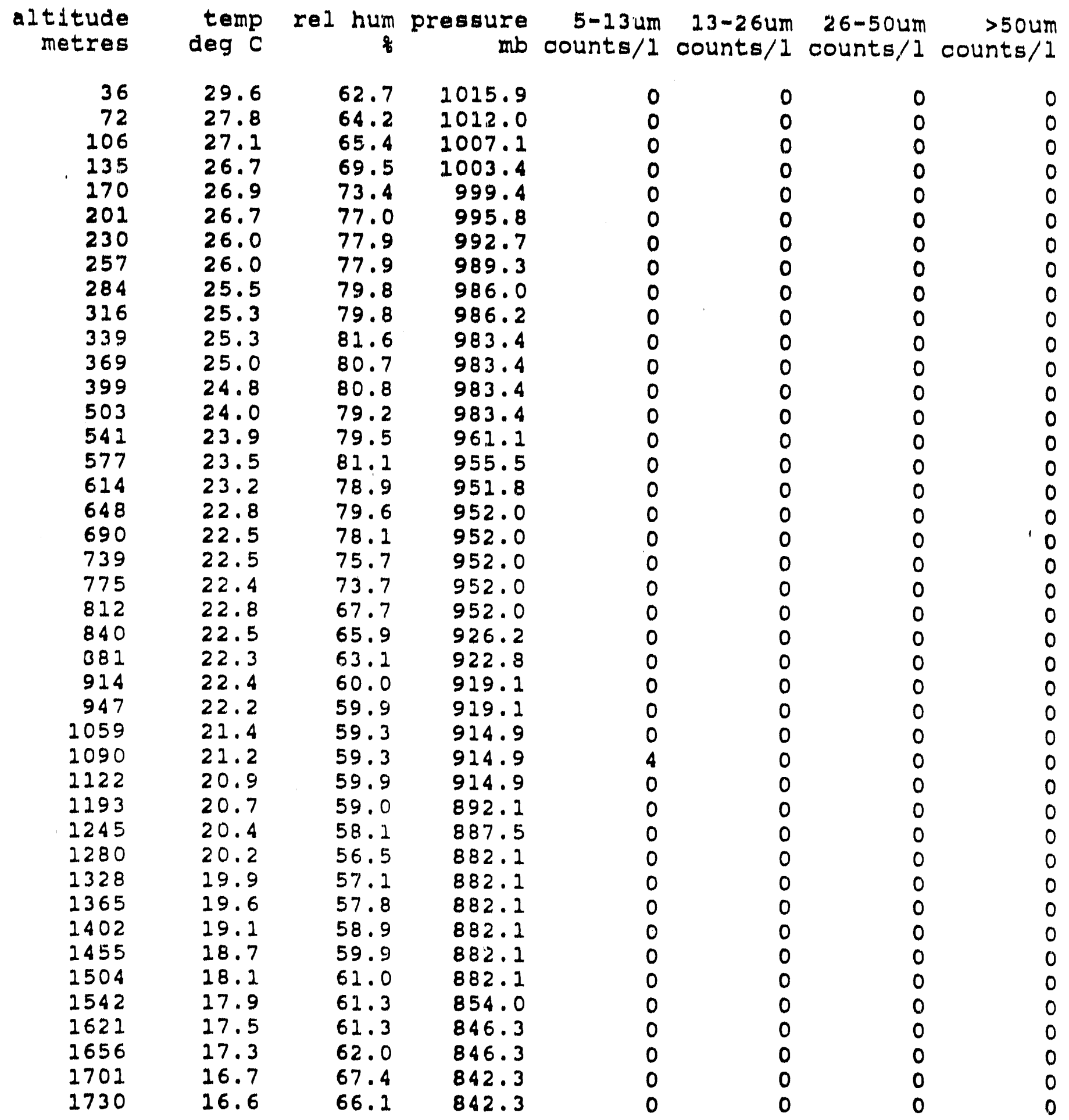




\section{JUL88}

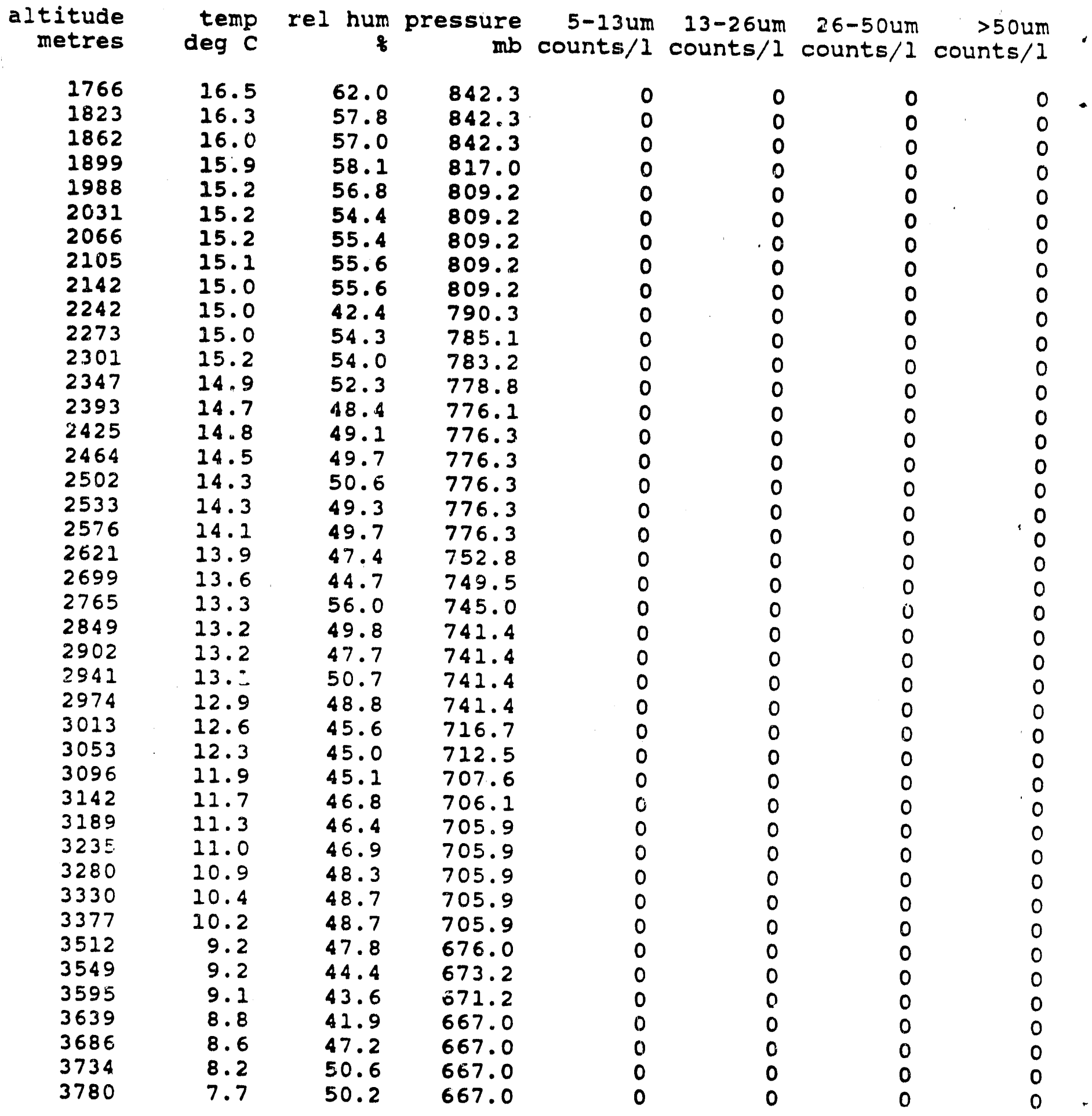




\section{JUL8 8}

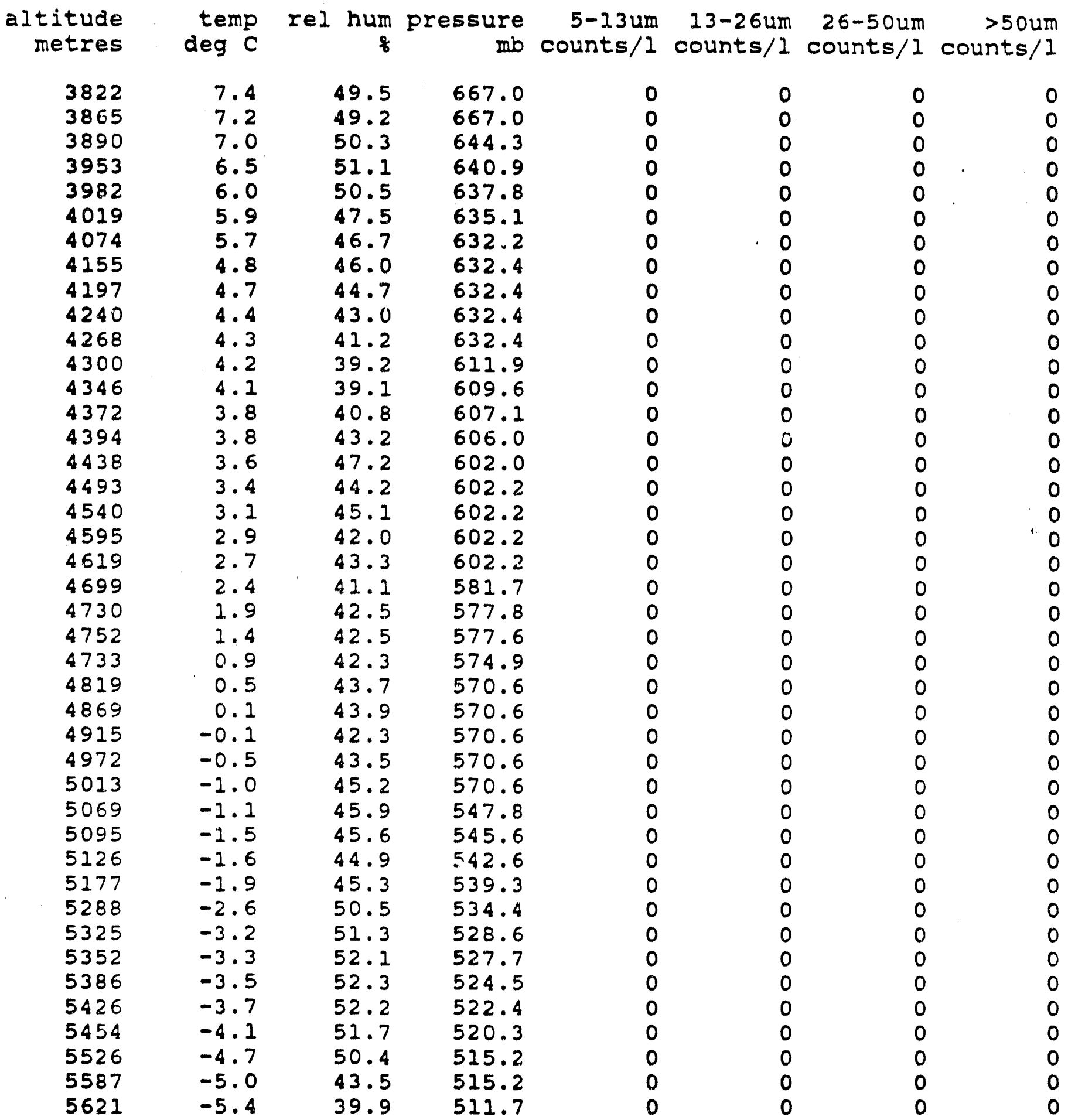




\section{JUL88}

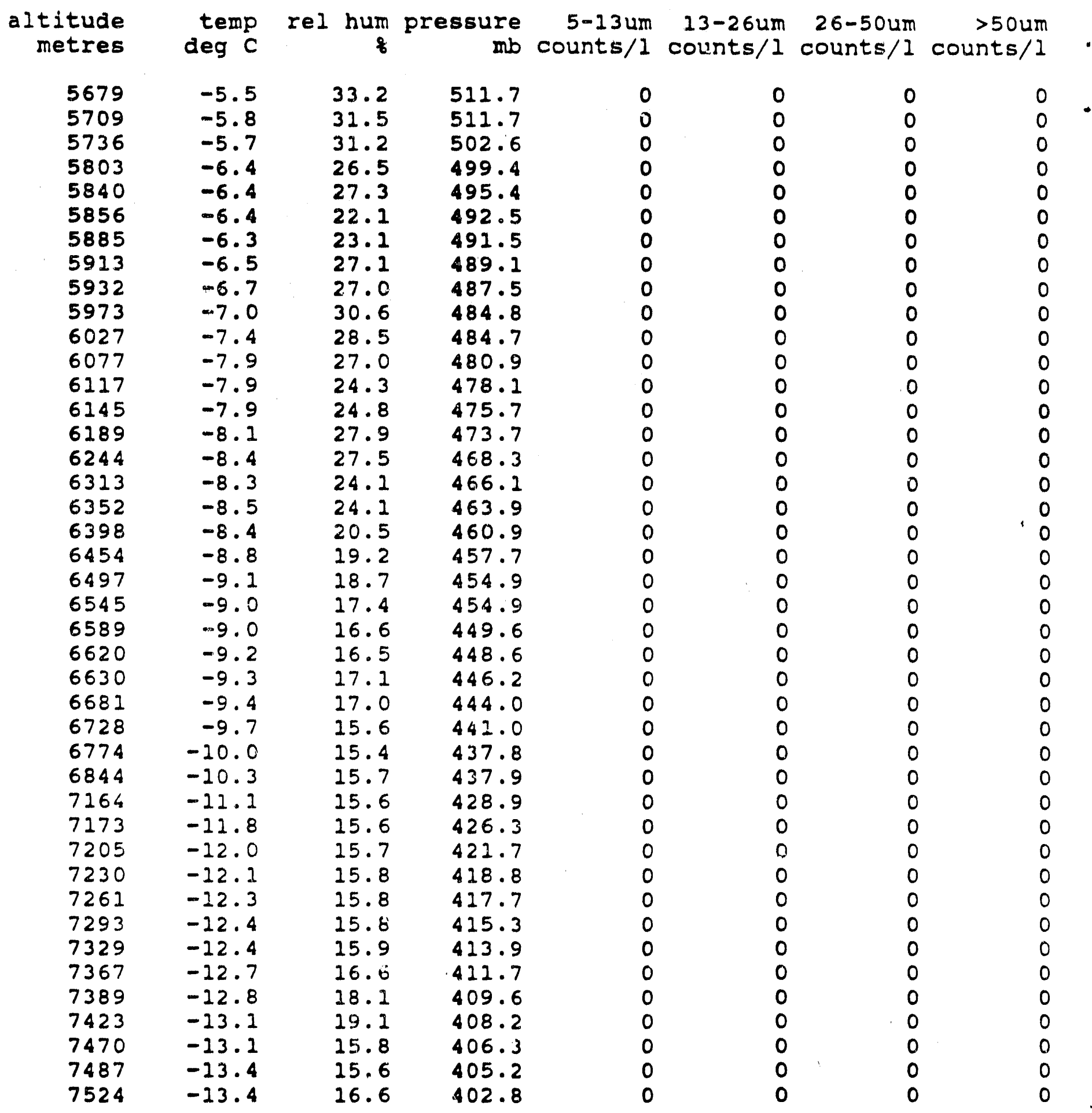




\section{JUL8 8}

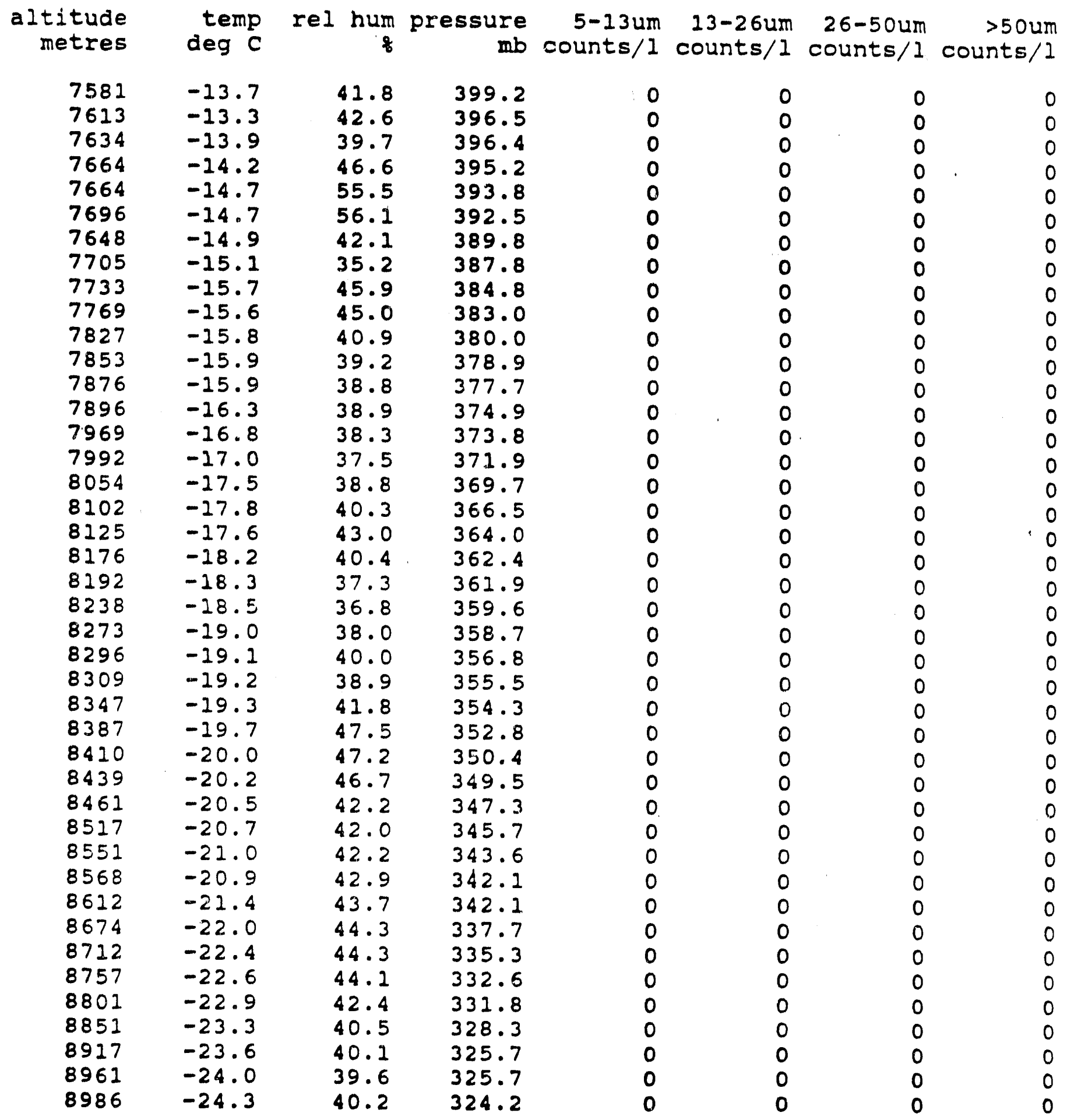




\section{JUL8 8}

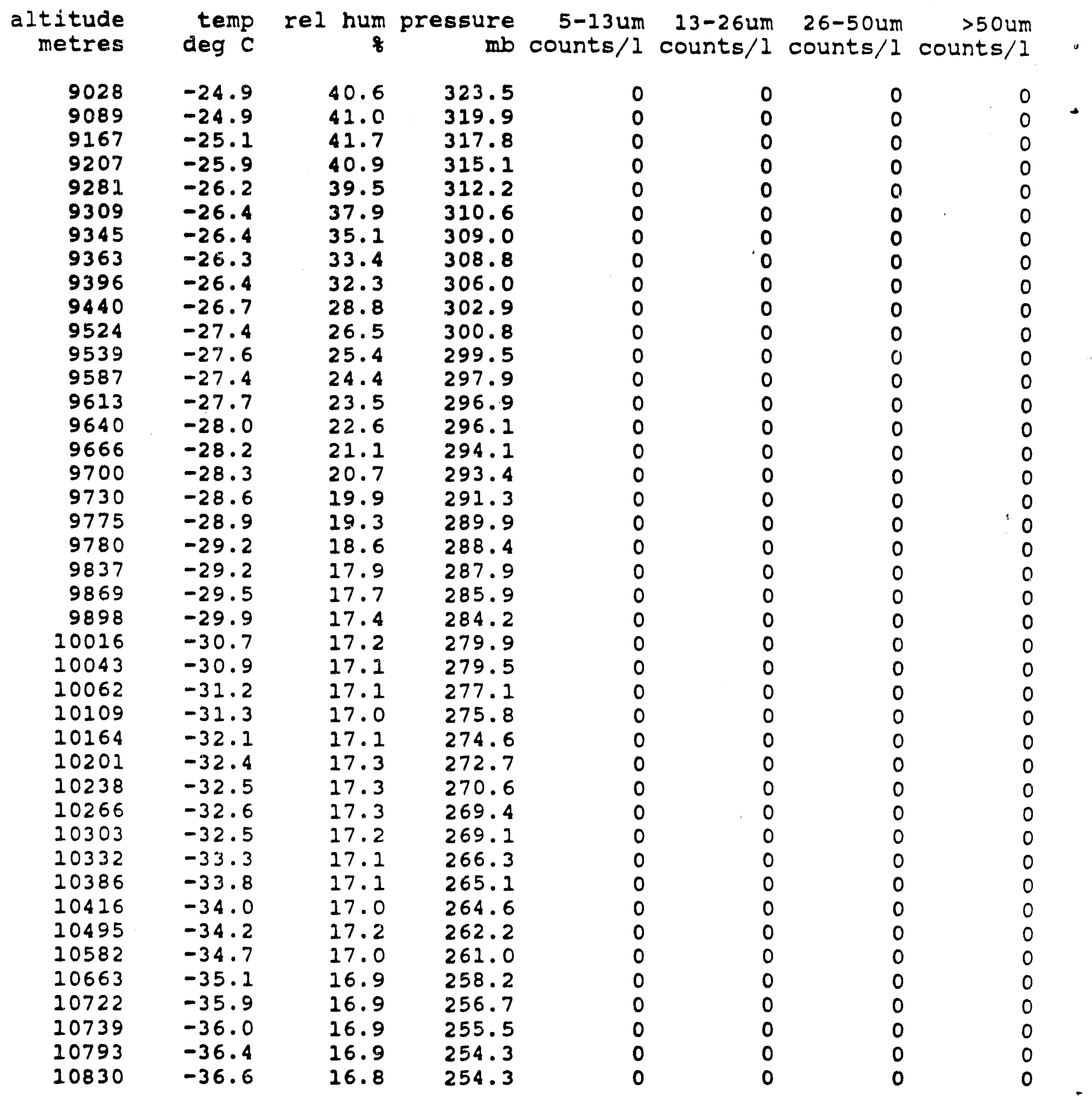




\section{JUL8 8}

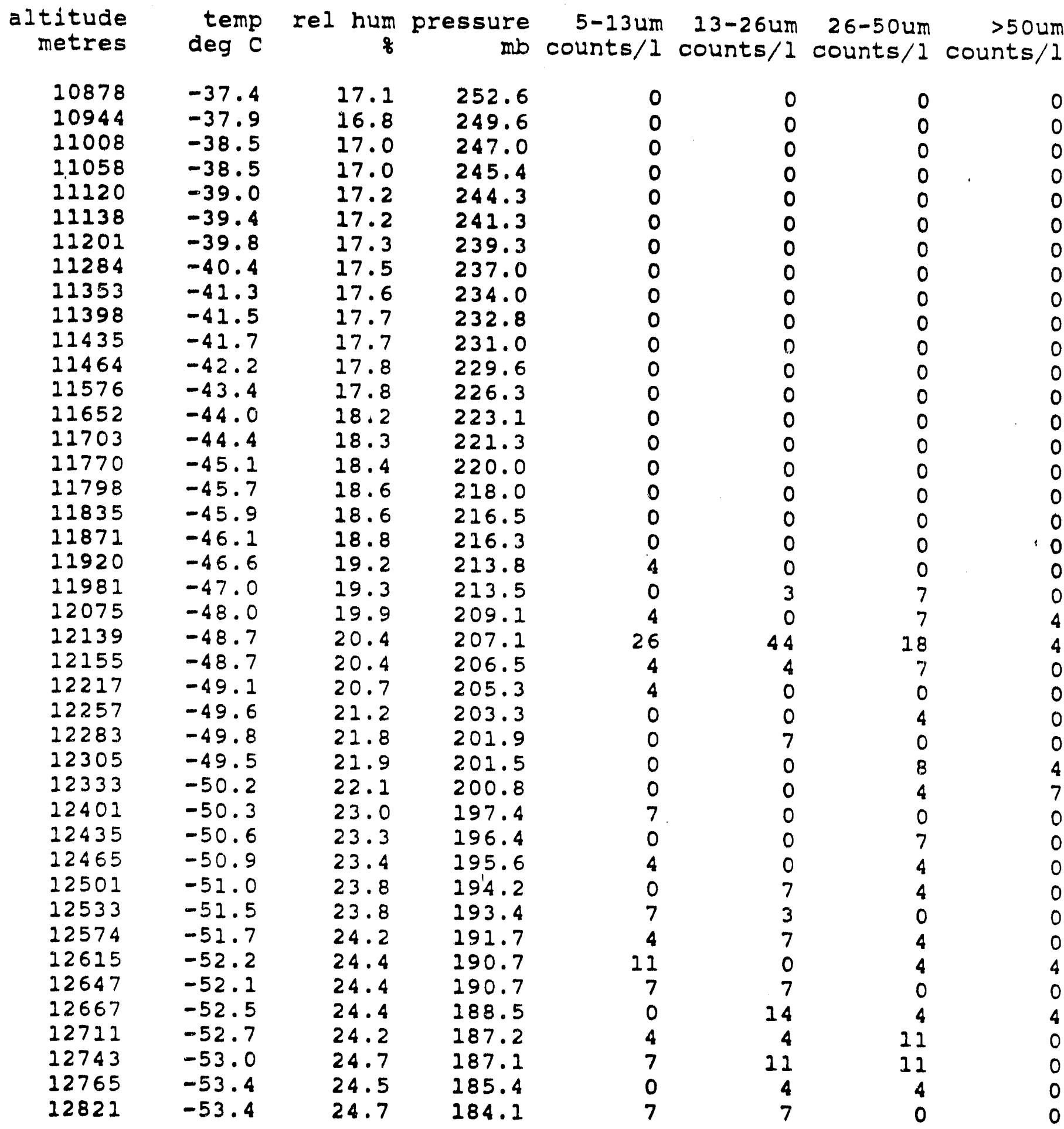




\section{JUL8 8}

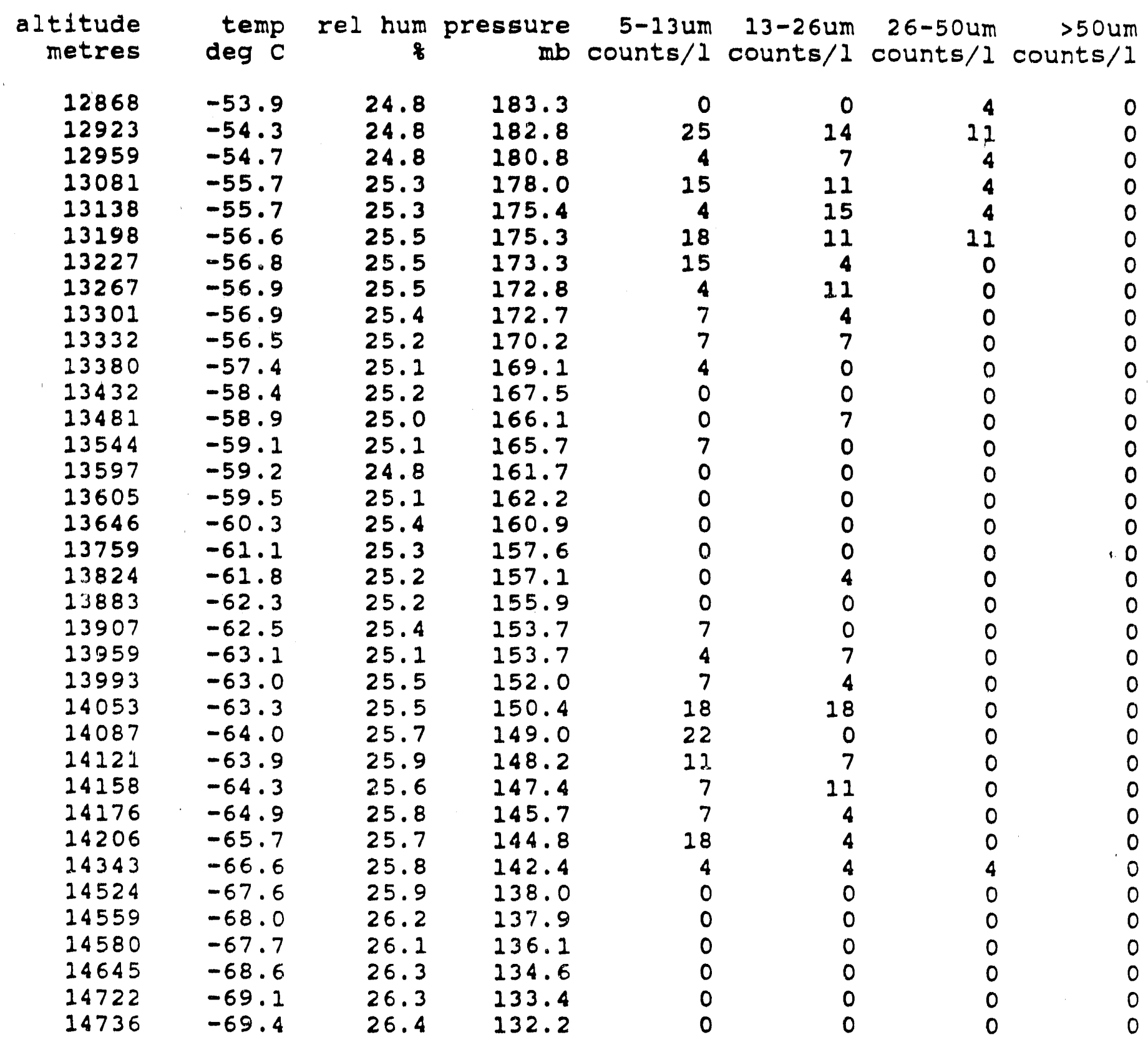




\section{APPENDIX B}

CALIFORNIA DATA 
BAUG8 8

$\begin{array}{rrrrr}\text { altitude } & \text { temp rel hum pressure } 5-13 \mathrm{um} 13-26 \mathrm{um} & 26-50 \mathrm{um} & >50 u m \\ \text { metres } & \text { deg } C & \text { mb counts } / 1 \text { counts } / 1 \text { counts } / 1 \text { counts } / 1 .\end{array}$

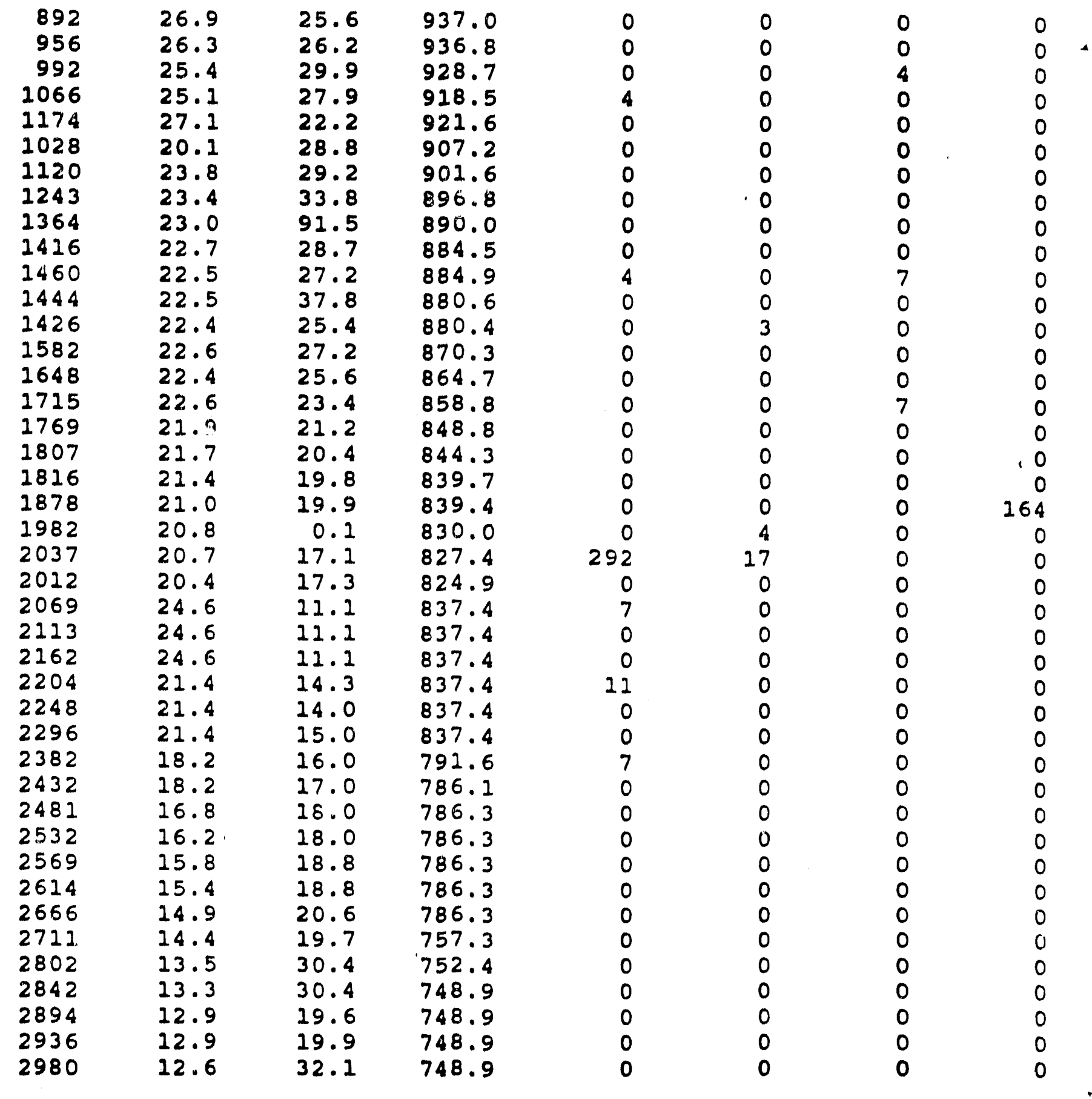


8AUG8 8

\begin{tabular}{|c|c|c|c|c|c|c|c|}
\hline $\begin{array}{l}\text { 1titude } \\
\text { metres }\end{array}$ & $\begin{array}{l}\text { temp } \\
\text { deg } C\end{array}$ & rel hum & $\begin{array}{r}\text { pressure } \\
\text { mb }\end{array}$ & $\begin{array}{c}5-13 \mathrm{um} \\
\text { counts/1 }\end{array}$ & $\begin{array}{c}13-26 u m \\
\text { counts } / 1\end{array}$ & $\begin{array}{c}26-50 u m \\
\text { counts/1 }\end{array}$ & $\begin{array}{r}>50 \text { un } \\
\text { counts } / 2\end{array}$ \\
\hline $\begin{array}{l}3236 \\
3139 \\
3161 \\
3203 \\
3249 \\
3300 \\
3350 \\
3389 \\
3436 \\
3488 \\
3539 \\
3596 \\
3699 \\
3773 \\
3814 \\
3874 \\
3921 \\
3972 \\
4014 \\
4061 \\
4112 \\
4151 \\
4193 \\
4236 \\
4289 \\
4341 \\
4518 \\
4566 \\
4607 \\
4656 \\
4688 \\
4741 \\
4778 \\
4815 \\
4863 \\
4907 \\
4960 \\
4997 \\
5047 \\
5148 \\
5200 \\
5236\end{array}$ & $\begin{array}{r}11.7 \\
11.4 \\
11.3 \\
10.9 \\
10.5 \\
10.1 \\
9.7 \\
9.2 \\
9.2 \\
9.2 \\
8.3 \\
7.9 \\
6.9 \\
8.6 \\
8.6 \\
8.6 \\
5.4 \\
5.0 \\
4.6 \\
4.2 \\
3.8 \\
3.3 \\
3.9 \\
2.9 \\
2.5 \\
1.9 \\
0.3 \\
2.4 \\
2.4 \\
2.4 \\
0.2 \\
0.4 \\
0.6 \\
0.2 \\
0.0 \\
-0.3 \\
-0.2 \\
-1.1 \\
-1.4 \\
-1.4 \\
-1.6 \\
-2.2 \\
-2.7 \\
-1.6\end{array}$ & $\begin{array}{l}19.8 \\
29.4 \\
19.2 \\
19.1 \\
19.5 \\
18.9 \\
19.1 \\
19.1 \\
19.1 \\
19.1 \\
19.2 \\
18.9 \\
18.1 \\
12.3 \\
12.3 \\
12.3 \\
17.0 \\
17.5 \\
17.5 \\
17.5 \\
18.0 \\
17.7 \\
17.9 \\
18.0 \\
26.9 \\
18.4 \\
11.4 \\
11.4 \\
16.8 \\
16.3 \\
16.1 \\
16.0 \\
16.0 \\
16.0 \\
16.1 \\
16.1 \\
16.0 \\
16.1 \\
16.2 \\
25.3 \\
16.4 \\
12.0\end{array}$ & $\begin{array}{l}726.0 \\
722.1 \\
717.3 \\
717.5 \\
713.4 \\
713.4 \\
713.4 \\
713.4 \\
713.4 \\
691.5 \\
689.0 \\
633.8 \\
683.8 \\
683.6 \\
683.6 \\
683.6 \\
683.6 \\
683.6 \\
650.8 \\
646.7 \\
642.1 \\
642.2 \\
638.5 \\
638.5 \\
638.5 \\
638.5 \\
610.4 \\
610.4 \\
610.4 \\
610.4 \\
610.4 \\
592.1 \\
5818.7 \\
586.1 \\
586.3 \\
582.3 \\
582.3 \\
582.3 \\
582.3 \\
563.1 \\
560.1 \\
565.5\end{array}$ & $\begin{array}{l}0 \\
0 \\
0 \\
0 \\
0 \\
0 \\
0 \\
0 \\
0 \\
0 \\
0 \\
0 \\
0 \\
4 \\
0 \\
0 \\
0 \\
0 \\
0 \\
0 \\
0 \\
0 \\
0 \\
0 \\
0 \\
0 \\
7 \\
0 \\
0 \\
0 \\
0 \\
0 \\
0 \\
0 \\
0 \\
0 \\
0 \\
0 \\
0 \\
0 \\
0 \\
3\end{array}$ & $\begin{array}{l}0 \\
0 \\
0 \\
0 \\
0 \\
0 \\
0 \\
0 \\
0 \\
0 \\
0 \\
0 \\
0 \\
0 \\
0 \\
0 \\
0 \\
0 \\
0 \\
0 \\
0 \\
0 \\
0 \\
0 \\
0 \\
0 \\
0 \\
0 \\
0 \\
0 \\
0 \\
0 \\
0 \\
0 \\
0 \\
0 \\
0 \\
3 \\
0 \\
0 \\
0 \\
0\end{array}$ & $\begin{array}{l}0 \\
0 \\
0 \\
0 \\
0 \\
0 \\
0 \\
0 \\
0 \\
0 \\
0 \\
0 \\
0 \\
0 \\
0 \\
0 \\
0 \\
0 \\
0 \\
0 \\
0 \\
0 \\
0 \\
0 \\
0 \\
0 \\
0 \\
0 \\
0 \\
0 \\
0 \\
0 \\
0 \\
0 \\
0 \\
0 \\
0 \\
0 \\
0 \\
0 \\
0 \\
0\end{array}$ & . \\
\hline
\end{tabular}




\section{BAUGB8}

altitude metres

$\begin{array}{llll}5275 & -3.1 & 16.5 & 565.5 \\ 5316 & -3.2 & 16.6 & 565.5 \\ 5359 & -3.5 & 16.5 & 565.5 \\ 5408 & -3.9 & 16.6 & 565.5 \\ 5456 & -4.4 & 16.6 & 565.5 \\ 5512 & -4.4 & 16.5 & 540.4 \\ 5604 & -5.3 & 16.7 & 536.1 \\ 5608 & -5.3 & 16.6 & 533.1 \\ 5384 & -5.4 & 16.3 & 529.9 \\ 5490 & -5.8 & 16.1 & 526.3 \\ 5784 & -7.2 & 16.2 & 524.1 \\ 5832 & -6.7 & 16.1 & 524.1 \\ 5880 & -7.2 & 16.2 & 524.1 \\ 5933 & -7.7 & 16.1 & 524.1 \\ 6417 & -12.5 & 16.4 & 466.1 \\ 6685 & -12.9 & 16.8 & 461.4 \\ 6814 & -13.2 & 16.7 & 458.0 \\ 6624 & -13.7 & 16.5 & 455.4 \\ 6896 & -14.6 & 19.3 & 447.2 \\ 6929 & -14.8 & 17.1 & 449.7 \\ 6897 & -14.8 & 17.1 & 446.4 \\ 6818 & -15.5 & 17.7 & 442.0 \\ 7225 & -16.7 & 15.1 & 435.5 \\ 7265 & -17.8 & 18.7 & 427.7 \\ 7320 & -18.4 & 32.7 & 425.2 \\ 7447 & -19.0 & 18.9 & 420.0 \\ 7482 & -19.2 & 18.7 & 417.8 \\ 7509 & -19.6 & 19.1 & 415.8 \\ 7580 & -20.2 & 18.9 & 411.1 \\ 7653 & -20.6 & 18.9 & 407.1 \\ 7705 & -21.4 & 19.1 & 401.2 \\ 7724 & -21.5 & 19.2 & 401.6 \\ 7783 & -22.0 & 19.2 & 399.1 \\ 7751 & -22.2 & 19.3 & 399.5 \\ 7800 & -21.7 & 13.9 & 403.5 \\ 7860 & -22.1 & 14.0 & 402.4 \\ 7790 & -23.0 & 19.9 & 393.4 \\ 8061 & -23.2 & 23.3 & 392.6 \\ 8106 & -23.7 & 20.2 & 390.0 \\ 8033 & -23.7 & 19.8 & 388.7 \\ 8048 & -23.8 & 19.8 & 386.4 \\ 8228 & -25.6 & 21.8 & 374.1 \\ & & & \end{array}$

5-13um 13-26um 26-50um >50um. mb counts/1 counts/1 counts/1 counts/1

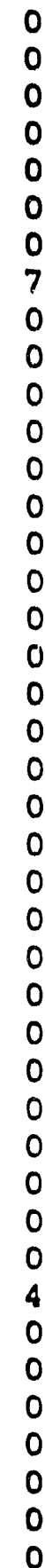

0
0
0
0
0
0
0
0
0
0
0
0
0
0
0
0
0
0
0
0
0
0
0
0
0
0
9
0
0
0
0
0
0
0
0
0
0
0
0
0
0
0
0
0
0
0
0
0
0
0
0
0
0
0
0

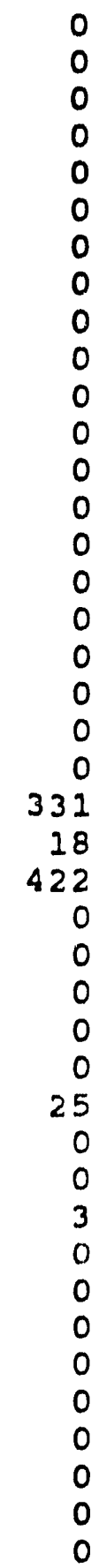




\section{AUGB 8}

altitude metres

8249

8268

8377

8420

8402

8478

8416

8425

8677

8651

8754

8786

8817

8830

8953

8851

9056

9103

8938

9502

9545

9553

9644

9.7.77

9794

9801

9990

9902

10097

10132

10159

10209

10244

9779

9886

10339

10299

10479

10674

10582

10801

20824 temp deg $C$

$-25.5$

$-25.5$

$-25.7$

$-25.9$

$-26.0$

$-26.5$

$-27.2$

$-27.4$

$-28.1$

$-28.1$

$-28.6$

$-28.9$

$-29.4$

$-29.5$

$-30.7$

$-15.3$

$-31.9$

$-32.3$

$-33.0$

$-34.0$

$-35.7$

$-36.2$

$-36.2$

$-36.8$

$-36.6$

$-37.4$

$-38.8$

$-39.4$

$-40.0$

$-40.0$

$-40.3$

$-40.4$

$-40.7$

$-41.1$

$-41.2$

$-41.4$

$-41.7$

$-42.3$

$-43.7$

$-43.8$

$-44.9$

$-45.1$ rel hum

20.0

18.6

18.8

26.5

18.5

18.8

19.0

18.9

18.9

17.5

19.0

19.0

19.2

19.2

19.1

81.6

19.3

19.5

20.0

21.0

21.2

19.3

16.1

19.2

16.6

19.2

19.6

19.8

19.8

19.9

19.9

19.7

19.6

19.4

20.0

22.5

19.7

30.3

20.1

27.6

20.3

20.1 pressure mb counts

5-13um

13-26um 26-50um counts/1 counts/1 counts/1

373.0
372.0

369.3

368.1

365.9

364.1

360.0

358.3

353.0

352.9

349.7

348.7

346.2

344.7

339.4

170.2

334.4

331.7

320.0

315.0

308.8

306.2

307.5

302.4

303.9

299.1

291.6

289.2

289.1

287.0

285.0

284.0

281.9

280.7

279.0

278.1

275.5

274.3

266.5

264.3

259.3

257.8

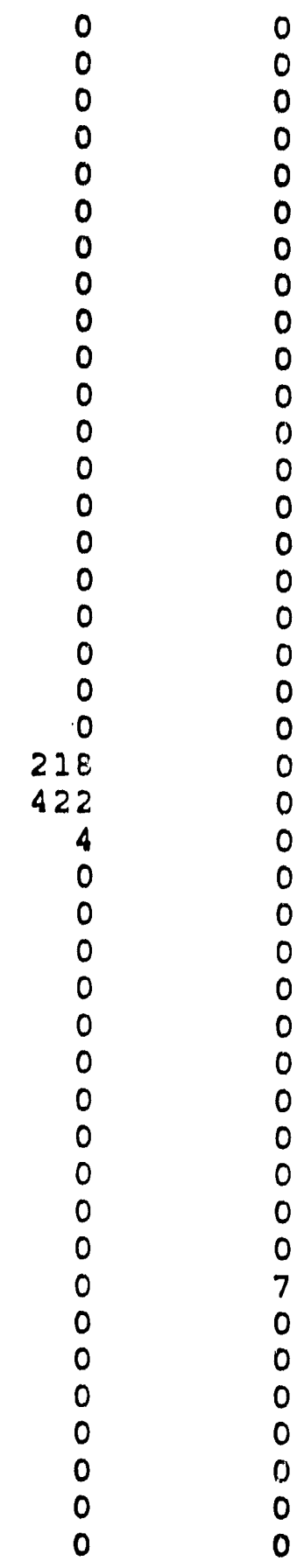

$\begin{array}{cc}0 & 0 \\ 0 & 0 \\ 0 & 0 \\ 0 & 0 \\ 0 & 0 \\ 0 & 0 \\ 0 & 0 \\ 0 & 0 \\ 0 & 0 \\ 0 & 0 \\ 0 & 0 \\ 0 & 0 \\ 0 & 0 \\ 0 & 0 \\ 0 & 0 \\ 0 & 0 \\ 0 & 0 \\ 0 & 0 \\ 0 & 0 \\ 0 & 0 \\ 160 & 0 \\ 0 & 0 \\ 0 & 0 \\ 0 & 0 \\ 0 & 0 \\ 0 & 0 \\ 0 & 0 \\ 0 & 0 \\ 0 & 0 \\ 0 & 0 \\ 0 & 0 \\ 0 & 0 \\ 0 & 0 \\ 0 & 0 \\ 0 & 0 \\ 0 & 0 \\ 0 & 0 \\ 0 & 0 \\ 0 & 0 \\ 0 & 0 \\ 0 & 0 \\ 0 & 0\end{array}$ 
8AUG88

altitude metres

$\begin{array}{llll}10867 & -45.3 & 20.1 & 256.3 \\ 10813 & -45.4 & 20.1 & 254.8 \\ 10943 & -45.8 & 20.3 & 253.3 \\ 10958 & -46.1 & 20.3 & 251.6 \\ 10949 & -46.1 & 20.3 & 251.6 \\ 10929 & -46.5 & 20.3 & 250.0 \\ 11094 & -46.5 & 20.1 & 248.9 \\ 11333 & -46.7 & 20.1 & 246.3 \\ 11217 & -47.5 & 20.2 & 244.0 \\ 11272 & -47.7 & 20.2 & 242.7 \\ 11199 & -48.1 & 20.2 & 240.4 \\ 11284 & -48.3 & 20.1 & 238.6 \\ 11407 & -48.7 & 20.2 & 236.2 \\ 11.473 & -48.8 & 20.2 & 233.7 \\ 11609 & -49.3 & 20.2 & 229.7 \\ 11739 & -50.1 & 20.3 & 227.1 \\ 11753 & -50.0 & 20.5 & 225.8 \\ 11837 & -49.7 & 20.5 & 223.2 \\ 11916 & -50.0 & 20.3 & 220.5 \\ 11938 & -50.1 & 20.4 & 218.7 \\ 12000 & -50.2 & 20.6 & 217.6 \\ 12026 & -5.0 .3 & 20.4 & 217.6 \\ 12038 & -50.3 & 20.4 & 216.2 \\ 12087 & -50.2 & 20.4 & 215.2 \\ 12127 & -50.2 & 20.4 & 214.2 \\ 12150 & -51.0 & 20.4 & 212.4 \\ 12187 & -51.1 & 20.3 & 211.1 \\ 12251 & -51.2 & 20.9 & 209.9 \\ 12286 & -51.8 & 20.6 & 207.7 \\ 12363 & -51.9 & 20.3 & 205.3 \\ 12482 & -52.1 & 20.5 & 201.9 \\ 12560 & -51.9 & 20.4 & 200.4 \\ 12610 & -51.8 & 20.3 & 198.6 \\ 12649 & -51.5 & 20.4 & 197.2 \\ 12680 & -51.4 & 20.4 & 195.8 \\ 12113 & -51.0 & 20.3 & 195.1 \\ 12757 & -51.3 & 20.4 & 193.7 \\ 12784 & -51.4 & 21.0 & 192.7 \\ 12805 & -51.7 & 20.4 & 191.1 \\ 12847 & -51.8 & 25.6 & 189.7 \\ 12926 & -51.5 & 20.4 & 188.7 \\ 12962 & -51.7 & 20.4 & 187.3\end{array}$

temp rel hum pressure $\operatorname{deg} C$ mb counts

5-13um 13-26um 26-50um $>50 u m$ mb counts/1 counts/1 counts/1 counts/1 


\section{BAUG8 8}

altitude metres

\begin{tabular}{|c|c|c|c|c|}
\hline $\begin{array}{l}13034 \\
13067 \\
13192 \\
13245 \\
13262 \\
13288 \\
13298 \\
13371 \\
13367 \\
13432 \\
13490 \\
13546 \\
13584 \\
13619 \\
13656 \\
13687 \\
13815 \\
13857 \\
13869 \\
13907 \\
13944 \\
14006 \\
14050 \\
14079 \\
14133 \\
14191 \\
14282 \\
14264 \\
14329 \\
14352 \\
14415 \\
14460 \\
14505 \\
14585 \\
14636 \\
14638 \\
14712 \\
14703 \\
14764 \\
14818 \\
14833 \\
14885\end{array}$ & $\begin{array}{l}-52.0 \\
-52.0 \\
-51.4 \\
-52.0 \\
-51.7 \\
-52.1 \\
-52.4 \\
-52 \\
-51.9 \\
-52.3 \\
-52.5 \\
-52.5 \\
-52.9 \\
-52.8 \\
-53.0 \\
-53.2 \\
-53.3 \\
-53.9 \\
-54.4 \\
-54.2 \\
-53.9 \\
-53.9 \\
-53.9 \\
-53.7 \\
-53.9 \\
-53.9 \\
-54.2 \\
-54.1 \\
-54.6 \\
-54.3 \\
-55.1 \\
-55.2 \\
-55.2 \\
-55.5 \\
-55.4 \\
-56.0 \\
-56.1 \\
-56.0 \\
-55.9 \\
-56.3 \\
-56.2 \\
-56.3 \\
-56.5\end{array}$ & $\begin{array}{l}20.1 \\
20.2 \\
20.2 \\
20.1 \\
20.0 \\
20.0 \\
20.1 \\
20.1 \\
20.1 \\
20.1 \\
20.0 \\
20.1 \\
19.9 \\
20.0 \\
20.2 \\
20.1 \\
20.2 \\
20.2 \\
19.9 \\
20.2 \\
20.1 \\
20.2 \\
19.8 \\
20.1 \\
25.1 \\
20.2 \\
19.9 \\
19.9 \\
20.1 \\
19.8 \\
20.1 \\
20.1 \\
19.9 \\
20.1 \\
20.1 \\
19.9 \\
20.1 \\
20.2 \\
19.9 \\
19.9 \\
19.9 \\
19.9\end{array}$ & 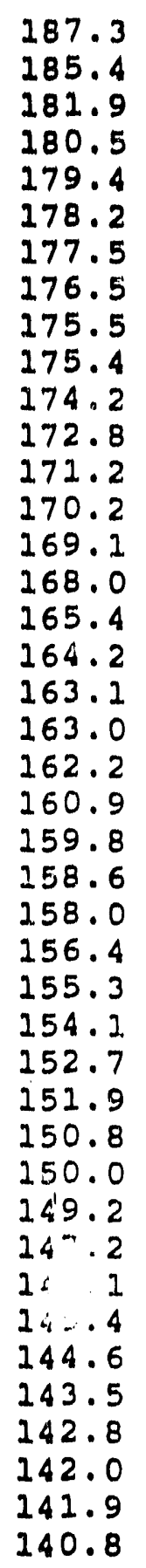 & $\begin{array}{l}0 \\
0 \\
0 \\
0 \\
0 \\
0 \\
0 \\
0 \\
0 \\
0 \\
0 \\
0 \\
0 \\
0 \\
0 \\
0 \\
0 \\
0 \\
0 \\
0 \\
0 \\
0 \\
0 \\
0 \\
0 \\
0 \\
0 \\
0 \\
0 \\
0 \\
0 \\
0 \\
0 \\
0 \\
0 \\
0 \\
0 \\
0 \\
0 \\
0 \\
0 \\
0\end{array}$ \\
\hline
\end{tabular}

temp rel hum pressure deg $C$

8

$5-13 u m$ re $13-26 u m$
mb counts/1 counts/1

26-50um counts/1
$>50 \mathrm{um}$ counts/1

0
0
0
0
0
0
0
0
0
0
0
0
0
0
0
0
0
0
0
0
0
0
0
0
0
0
0
0
0
0
0
0
0
0
0
0
0
0
0
0
0
0

$\begin{array}{ll}0 & 0 \\ 0 & 0 \\ 0 & 0 \\ 0 & \\ 0 & 0 \\ 0 & 0 \\ 0 & 0 \\ 0 & 0 \\ 0 & 0 \\ 0 & 0 \\ 0 & 0 \\ 0 & 0 \\ 0 & 0 \\ 0 & 0 \\ 0 & 0 \\ 0 & 0 \\ 0 & 0 \\ 0 & 0 \\ 0 & 0 \\ 0 & 0 \\ 0 & 0 \\ 0 & 0 \\ 0 & 0 \\ 0 & 0 \\ 0 & 0 \\ 0 & 0 \\ 0 & 0 \\ 0 & 0 \\ 0 & 0 \\ 0 & 0 \\ 0 & 0 \\ 0 & 0 \\ 0 & 0 \\ 0 & 0 \\ 0 & 0 \\ 0 & 0 \\ 0 & 0 \\ 0 & 0 \\ 0 & 0 \\ 0 & 0 \\ 0 & 0 \\ 0 & 0\end{array}$


8AUG88

\begin{tabular}{|c|c|c|c|c|c|c|c|}
\hline $\begin{array}{r}\text { altitude } \\
\text { metres }\end{array}$ & $\begin{array}{l}\text { temp } \\
\text { deg } C\end{array}$ & rel hum & $\begin{array}{r}\text { pressure } \\
\text { mb }\end{array}$ & $\begin{array}{c}5-13 \text { um } \\
\text { counts/1 }\end{array}$ & $\begin{array}{c}13-26 u m \\
\text { counts } / 1\end{array}$ & $\begin{array}{c}26-50 u m \\
\text { counts/1 }\end{array}$ & $\begin{array}{r}>50 u m \\
\text { counts } / 1\end{array}$ \\
\hline 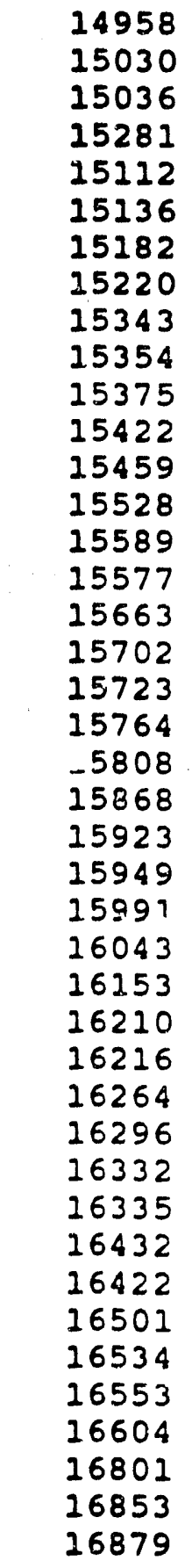 & $\begin{array}{l}-57.1 \\
-57.4 \\
-57.3 \\
-57.9 \\
-58.1 \\
-58.4 \\
-58.6 \\
-58.6 \\
-58.9 \\
-59.1 \\
-59.2 \\
-59.2 \\
-59.5 \\
-59.8 \\
-59.7 \\
-59.4 \\
-59.4 \\
-59.4 \\
-59.9 \\
-59.9 \\
-60.2 \\
-60.6 \\
-60.6 \\
-60.8 \\
-61.3 \\
-61.3 \\
-61.3 \\
-61.3 \\
-61.8 \\
-61.7 \\
-61.7 \\
-61.9 \\
-61.7 \\
-61.4 \\
-61.4 \\
-61.5 \\
-61.7 \\
-61.8 \\
-61.8 \\
-61.3 \\
-61.9 \\
-61.9\end{array}$ & $\begin{array}{l}20.0 \\
20.1 \\
18.3 \\
20.2 \\
18.2 \\
20.2 \\
20.3 \\
20.2 \\
20.3 \\
20.5 \\
20.3 \\
20.3 \\
20.3 \\
20.0 \\
20.6 \\
19.8 \\
19.6 \\
20.5 \\
23.7 \\
20.6 \\
23.9 \\
20.3 \\
24.2 \\
20.4 \\
20.3 \\
20.6 \\
20.4 \\
20.6 \\
20.6 \\
20.6 \\
20.4 \\
20.6 \\
20.4 \\
20.6 \\
20.4 \\
24.3 \\
24.3 \\
20.6 \\
23.7 \\
20.3 \\
20.6 \\
20.5\end{array}$ & $\begin{array}{l}138.8 \\
138.0 \\
137.5 \\
136.1 \\
136.0 \\
134.4 \\
133.6 \\
132.7 \\
132.0 \\
130.9 \\
130.3 \\
129.4 \\
128.7 \\
127.0 \\
126.0 \\
125.2 \\
124.4 \\
124.3 \\
125.8 \\
122.7 \\
121.5 \\
120.6 \\
119.9 \\
118.9 \\
118.2 \\
116.6 \\
115.8 \\
115.2\end{array}$ & $\begin{array}{l}0 \\
0 \\
0 \\
0 \\
0 \\
0 \\
0 \\
0 \\
0 \\
0 \\
0 \\
0 \\
0 \\
0 \\
0 \\
0 \\
0 \\
0 \\
0 \\
0 \\
0 \\
0 \\
0 \\
0 \\
0 \\
0 \\
0 \\
0 \\
0 \\
0 \\
0 \\
0 \\
0 \\
0 \\
0 \\
0 \\
0 \\
0 \\
0 \\
0 \\
0 \\
0\end{array}$ & $\begin{array}{l}0 \\
0 \\
0 \\
0 \\
0 \\
0 \\
0 \\
0 \\
0 \\
0 \\
0 \\
0 \\
0 \\
0 \\
0 \\
0 \\
0 \\
0 \\
0 \\
0 \\
0 \\
0 \\
0 \\
0 \\
0 \\
0 \\
0 \\
0 \\
0 \\
0 \\
0 \\
0 \\
0 \\
0 \\
0 \\
0 \\
0 \\
0 \\
0 \\
0 \\
0 \\
0\end{array}$ & $\begin{array}{l}0 \\
0 \\
0 \\
0 \\
0 \\
0 \\
0 \\
0 \\
0 \\
0 \\
0 \\
0 \\
0 \\
0 \\
0 \\
0 \\
0 \\
0 \\
0 \\
0 \\
0 \\
0 \\
0 \\
0 \\
0 \\
0 \\
0 \\
0 \\
0 \\
0 \\
0 \\
0 \\
0 \\
0 \\
0 \\
0 \\
0 \\
0 \\
0 \\
0 \\
0 \\
0\end{array}$ & $\begin{array}{l}0 \\
0 \\
0 \\
0 \\
0 \\
0 \\
0 \\
0 \\
0 \\
0 \\
0 \\
0 \\
0 \\
0 \\
0 \\
0 \\
0 \\
0 \\
0 \\
0 \\
0 \\
0 \\
0 \\
0 \\
0 \\
0 \\
0 \\
0 \\
0 \\
0 \\
0 \\
0 \\
0 \\
0 \\
0 \\
0 \\
0 \\
0 \\
0 \\
0 \\
0 \\
0\end{array}$ \\
\hline
\end{tabular}


8AUG8 8

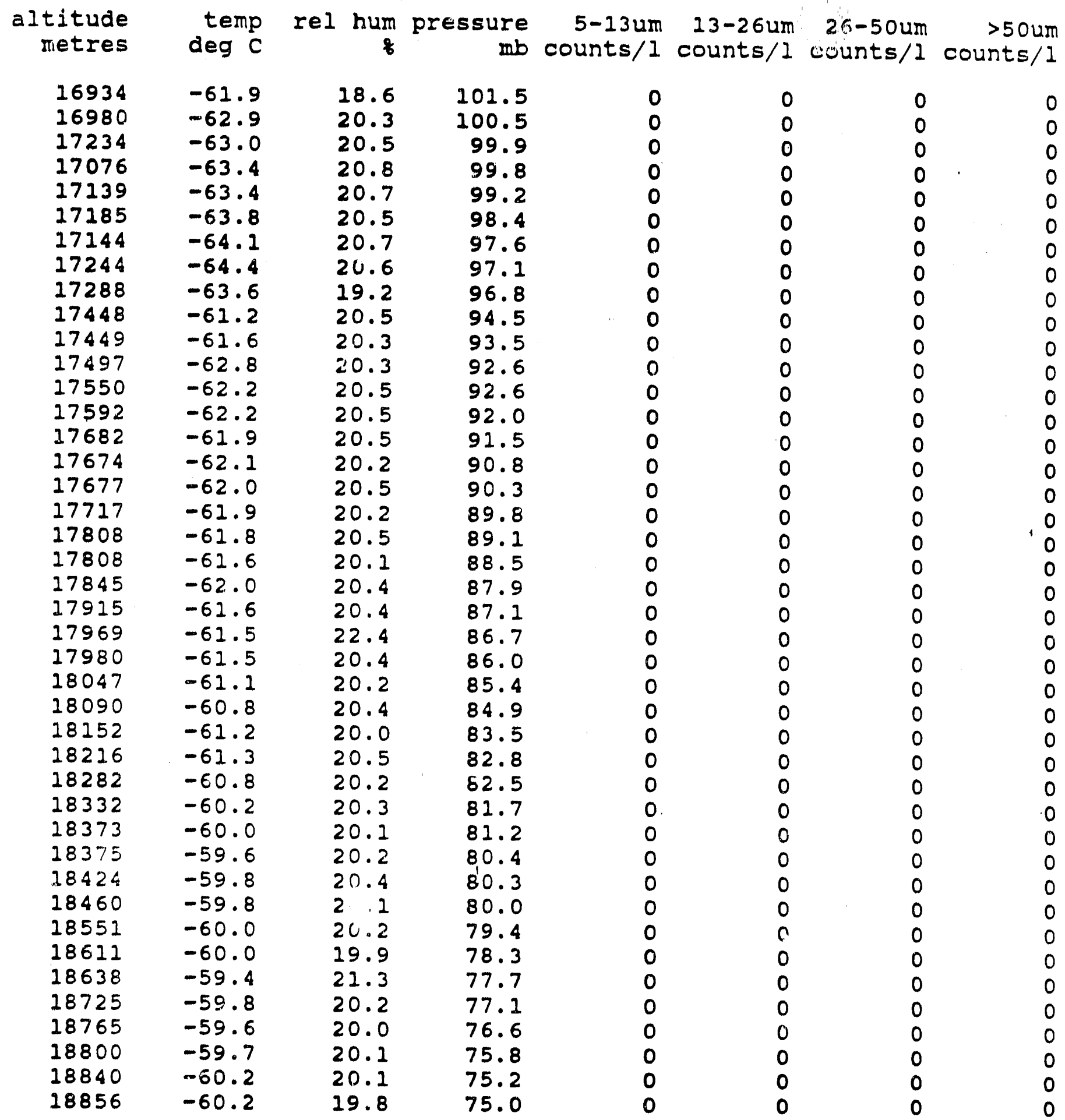




\section{BAUG8B}

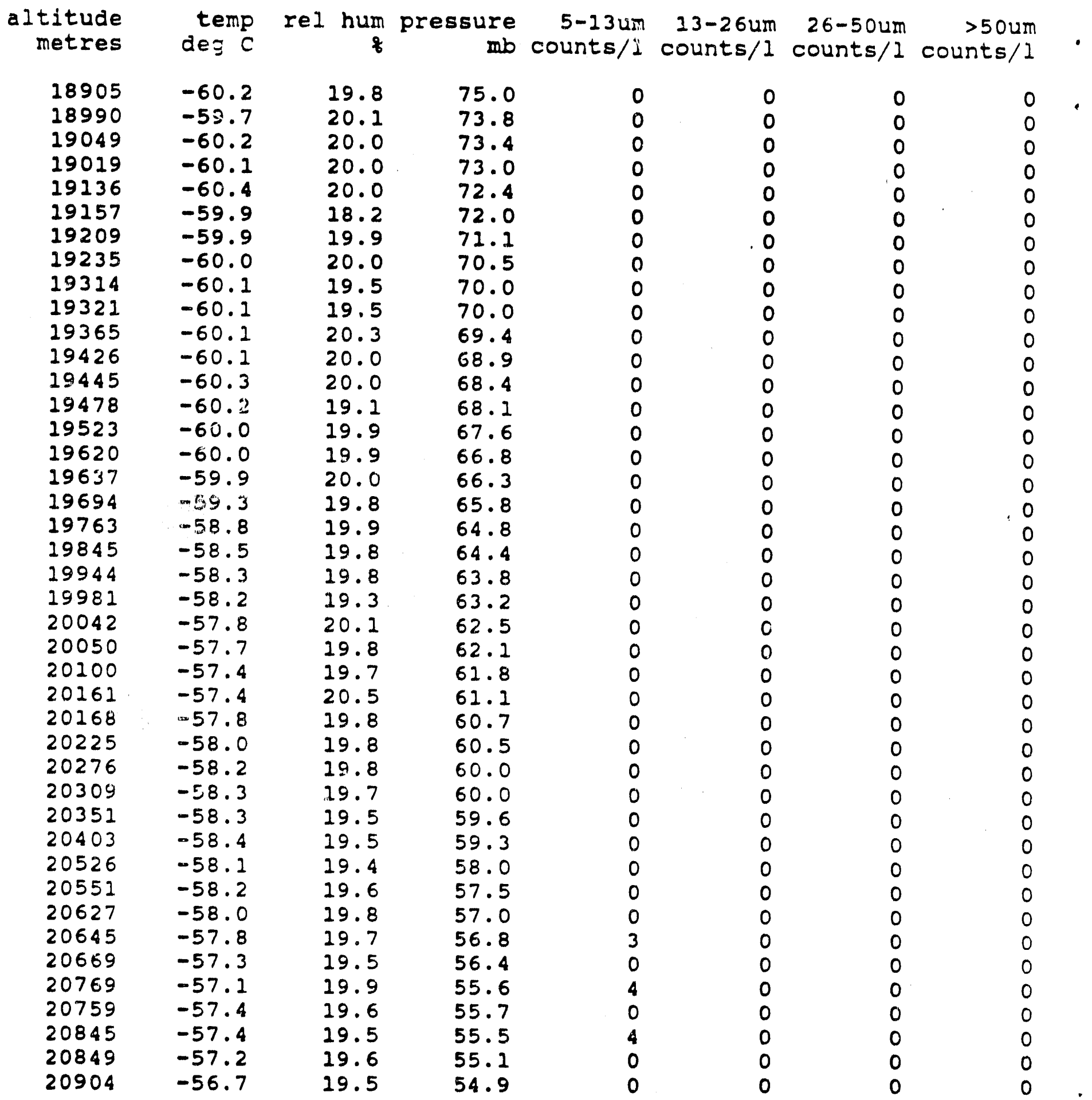


9AUG8B

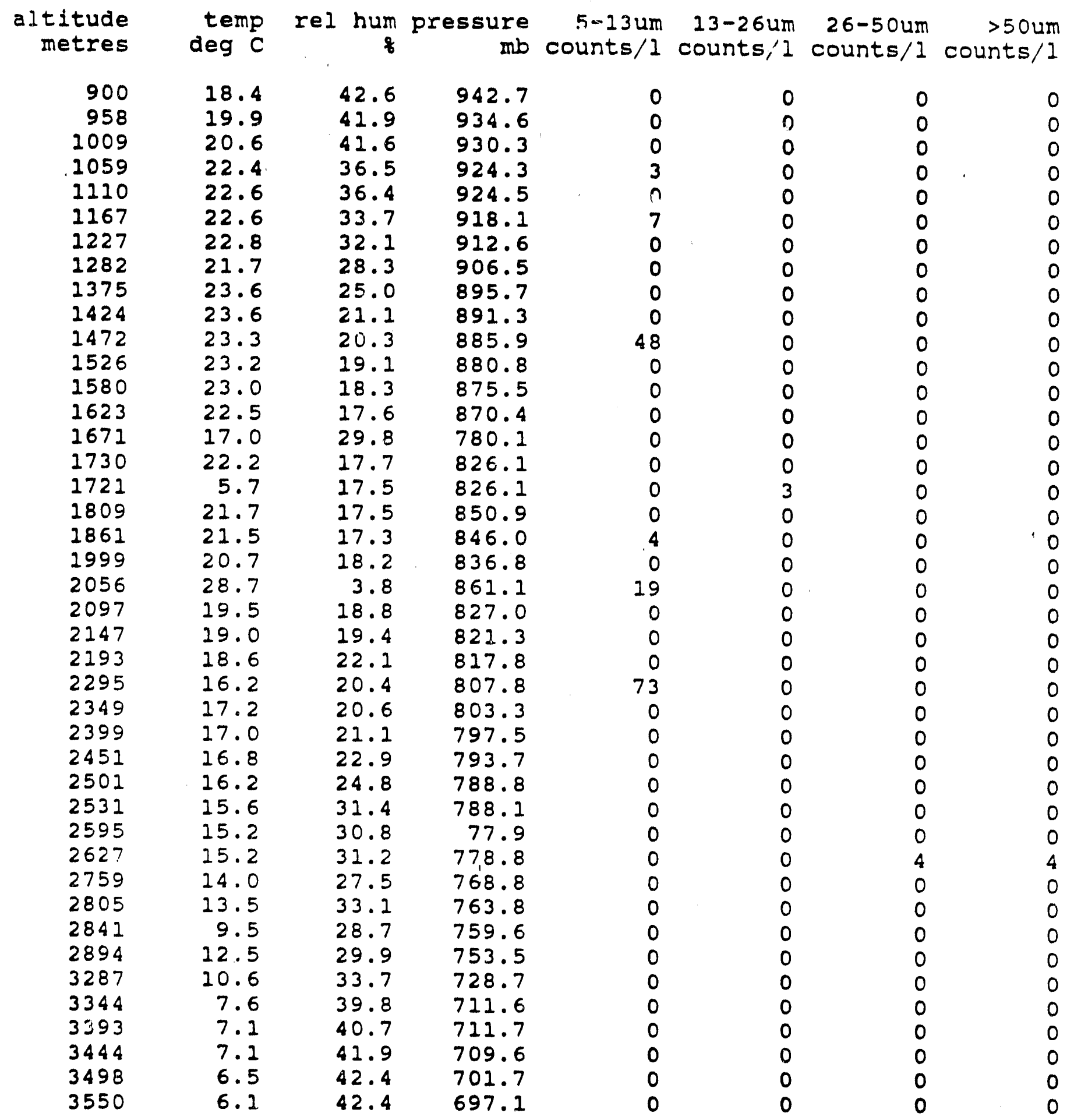




\section{AUG 88}

altitude metres

$\begin{array}{lrll}3601 & 5.6 & 42.4 & 692.7 \\ 3651 & 5.1 & 43.4 & 688.6 \\ 3709 & 4.9 & 32.3 & 684.7 \\ 3767 & 5.0 & 20.8 & 678.9 \\ 3810 & 5.1 & 17.0 & 674.3 \\ 3912 & 4.9 & 18.3 & 670.0 \\ 3962 & 4.3 & 15.8 & 663.5 \\ 4011 & 4.0 & 16.2 & 662.5 \\ 4067 & 3.8 & 15.7 & 654.2 \\ 4115 & 3.4 & 15.4 & 650.0 \\ 4170 & 3.6 & 15.4 & 645.2 \\ 4238 & 3.4 & 14.9 & 645.7 \\ 4278 & 3.4 & 15.4 & 640.7 \\ 4335 & 3.0 & 14.7 & 637.2 \\ 4381 & 2.7 & 14.9 & 633.8 \\ 4427 & 2.4 & 14.7 & 628.1 \\ 4485 & 2.0 & 14.9 & 623.9 \\ 4518 & 1.6 & 14.9 & 619.5 \\ 4581 & 1.1 & 14.8 & 616.3 \\ 4682 & 0.2 & 14.8 & 608.8 \\ 4749 & -0.5 & 14.9 & 602.1 \\ 4810 & -1.0 & 14.9 & 597.7 \\ 4858 & -1.4 & 14.9 & 593.3 \\ 4906 & -1.9 & 15.8 & 590.4 \\ 4960 & -2.3 & 15.9 & 586.1 \\ 5000 & -2.7 & 14.7 & 586.3 \\ 5053 & -2.7 & 14.9 & 584.9 \\ 5088 & -3.2 & 14.9 & 577.2 \\ 5168 & -3.7 & 14.6 & 574.2 \\ 5197 & -3.8 & 14.9 & 569.9 \\ 5263 & -4.1 & 14.8 & 567.2 \\ 5297 & -4.4 & 14.9 & 563.3 \\ 5344 & -4.4 & 14.9 & 559.9 \\ 5392 & -4.2 & 14.7 & 555.6 \\ 5449 & -4.4 & 14.9 & 551.9 \\ 5511 & -4.9 & 14.9 & 547.5 \\ 5562 & -5.3 & 14.5 & 543.5 \\ 5928 & -7.6 & 15.4 & 524.5 \\ 5977 & -8.4 & 15.6 & 521.7 \\ 6017 & -8.6 & 15.4 & 517.9 \\ 6057 & -9.1 & 15.6 & 514.7 \\ 6100 & -9.6 & 15.6 & 511.1\end{array}$

$\begin{array}{lll}5.6 & 42.4 & 692.7\end{array}$

$5.1 \quad 43.4 \quad 688.6$

$4.9 \quad 32.3 \quad 684.7$

$\begin{array}{lll}5.0 & 20.8 & 678.9\end{array}$

15.8

663.5

62.5

654.2

650.0

645.2

645.7

640.7

633.8

628.1

23.9

1.9 .5

16.3

08.8

502.1

97.7

593.3

586.1

586.3

584.9

577.2

50.9

567.2

563.3

559.9

55.6

51.9

543.5

524.5

521.7

514.7

511.1
590.4

temp rel hum pressure 5-13um 13-26um 26-50um deg $C$ \& mb counts/1 counts/1 counts/1 counts/1

$\begin{array}{ll}0 & 0 \\ 0 & 0 \\ 0 & 0 \\ 0 & 0 \\ 0 & 0 \\ 0 & 0 \\ 0 & 0 \\ 0 & 0 \\ 0 & 0 \\ 0 & 0 \\ 0 & 0 \\ 0 & 0 \\ 0 & 0 \\ 0 & 0 \\ 0 & 0 \\ 0 & 0 \\ 0 & 0 \\ 0 & 0 \\ 0 & 0 \\ 0 & 0 \\ 0 & 0 \\ 0 & 0 \\ 0 & 0 \\ 0 & 0 \\ 0 & 0 \\ 0 & 0 \\ 0 & 0 \\ 0 & 0 \\ 0 & 0 \\ 0 & 0 \\ 0 & 0 \\ 0 & 0 \\ 0 & 0 \\ 0 & 0 \\ 0 & 0 \\ 0 & 0 \\ 0 & 0 \\ 4 & 0 \\ 0 & 0 \\ 0 & 0 \\ 0 & 0 \\ 0 & 0 \\ & 0\end{array}$

0
0
0
0
0
0
0
0
0
0
0
0
0
0
0
0
0
0
0
0
0
0
0
0
0
0
0
0
0
0
0
0
0
0
0
0
0
4
0
0
0
0 


\section{AUG88}

$\begin{array}{rrrrr}\text { altitude temp rel hum pressure } 5-13 u m & 13-26 u m & 26-50 u m & >50 u m \\ \text { metres } & \text { deg } C & \text { mb counts/1 counts/1 counts/l counts } / 1\end{array}$

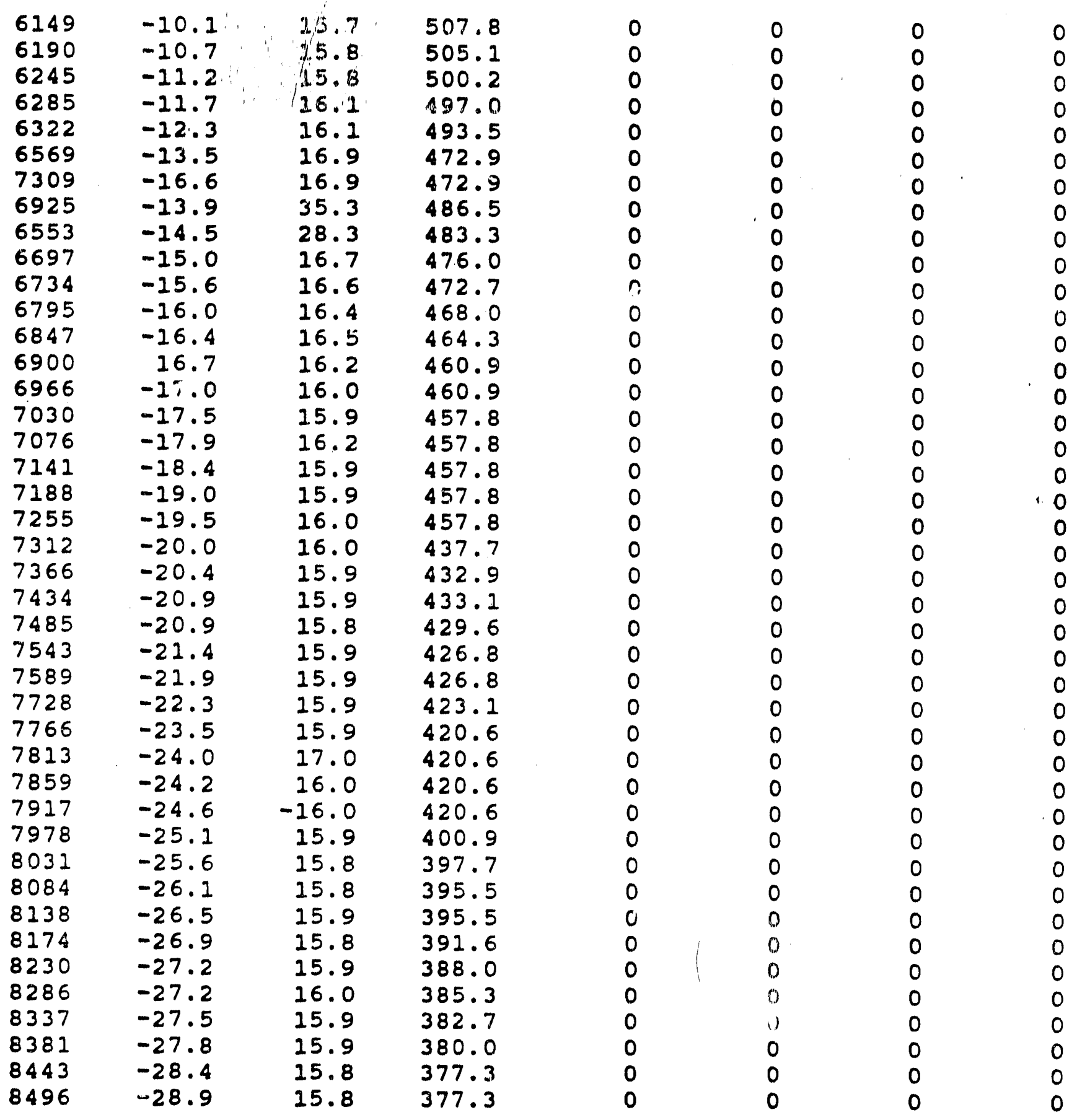


9AUG 88

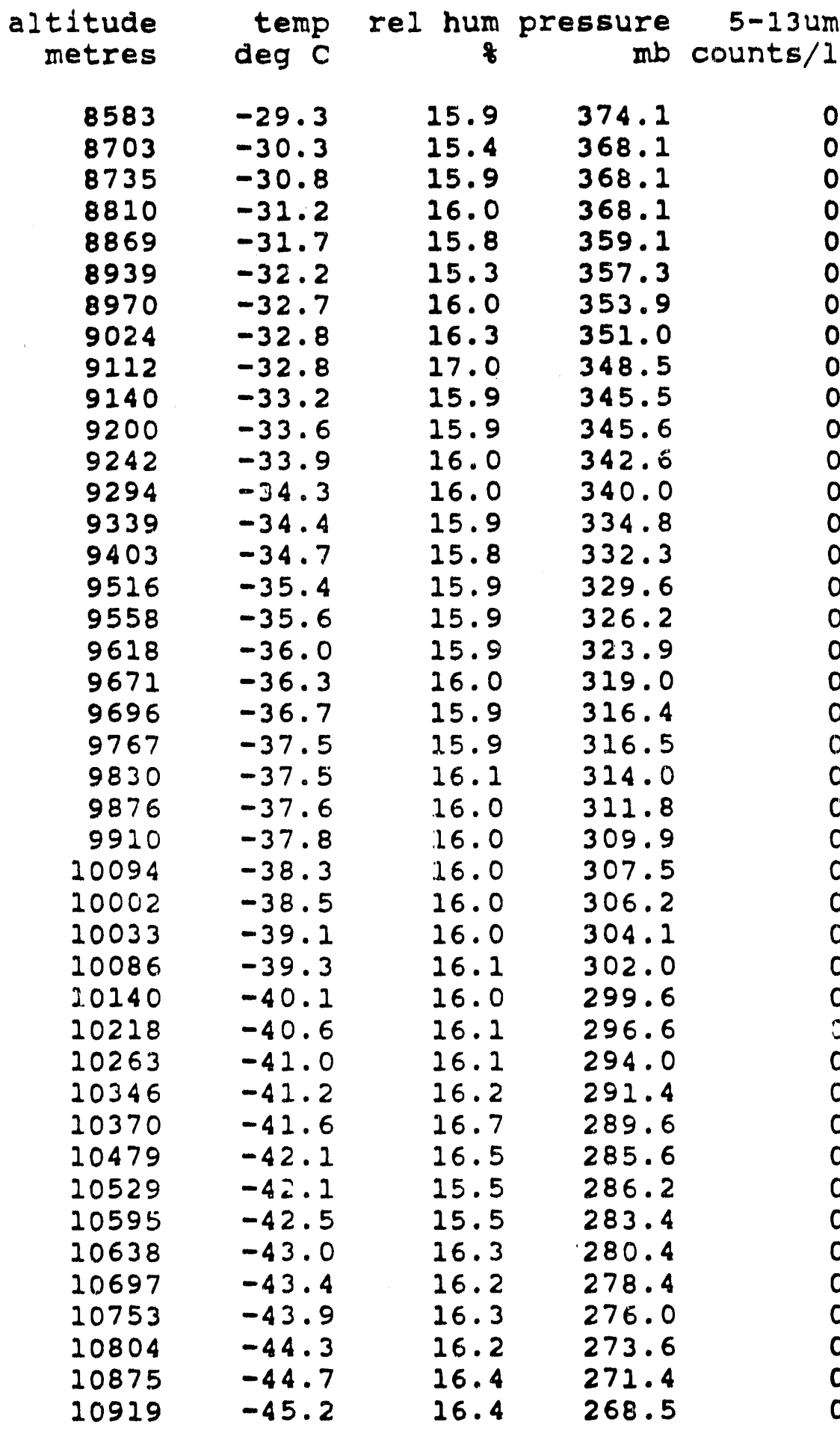

$13-26 u m$
$26-50 u m$
counts $/ 1$ counts $/ 1$ counts $/ 1$

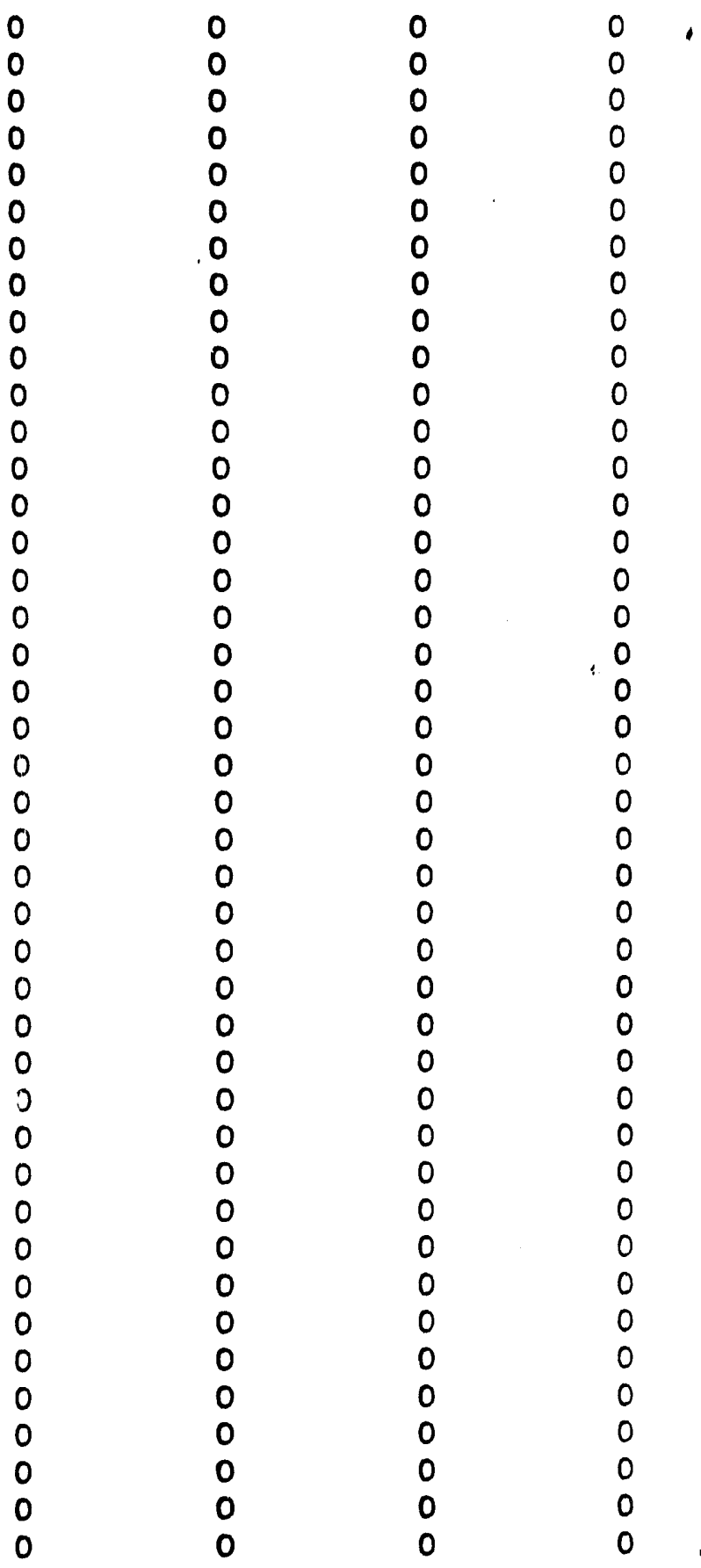




\section{AUG 88}

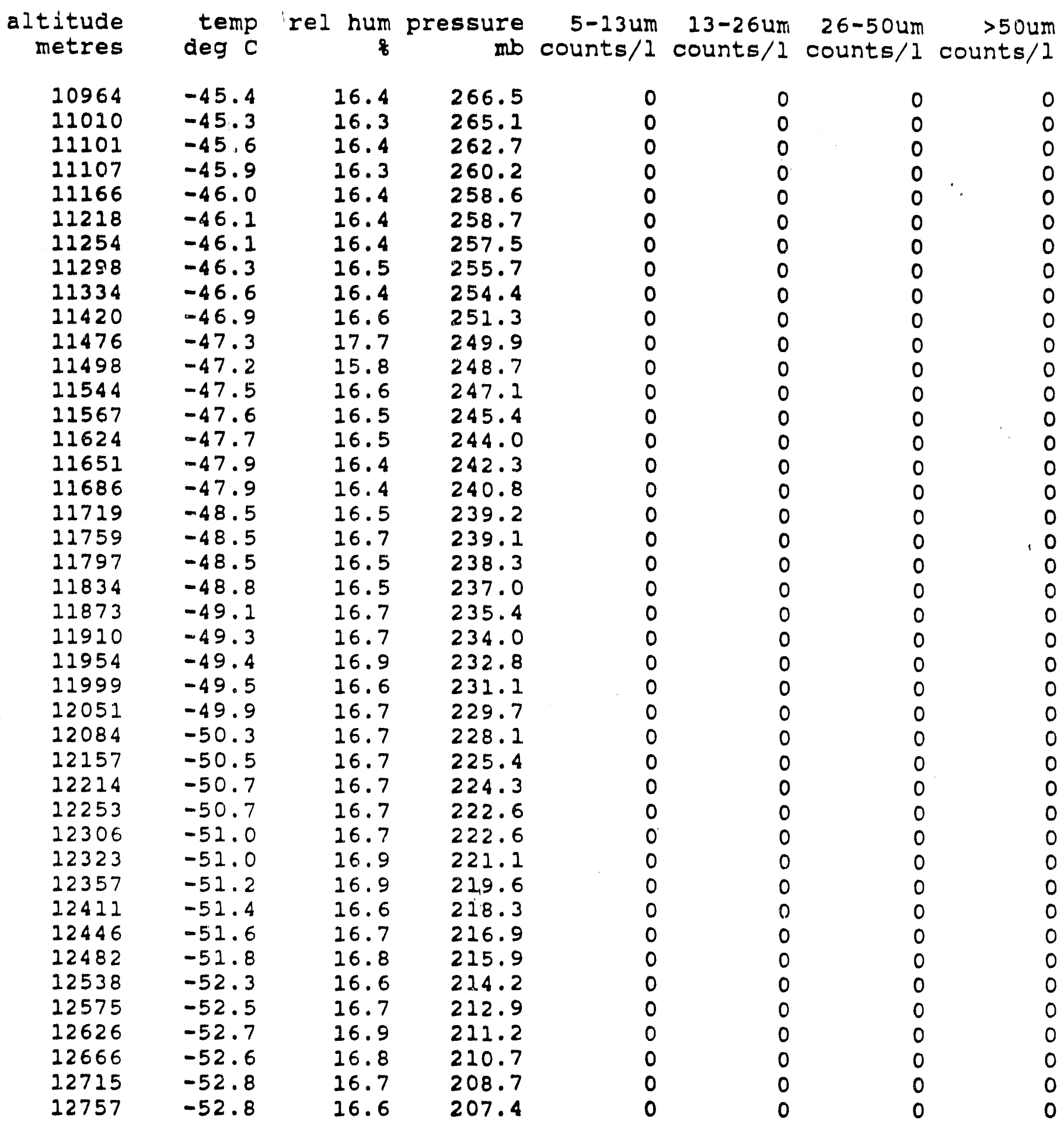




\section{AUG8 8}

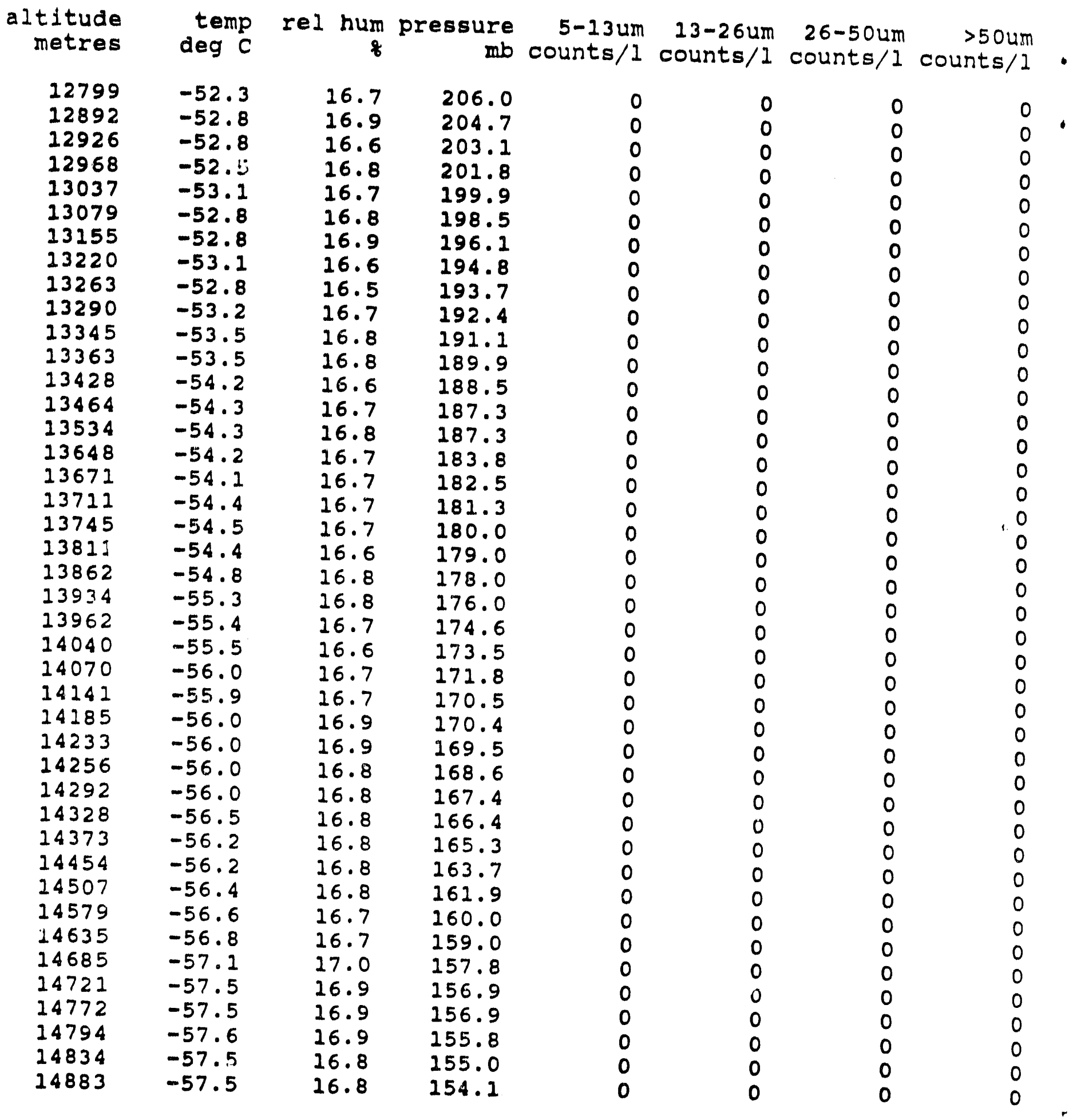




\section{AUG 88}

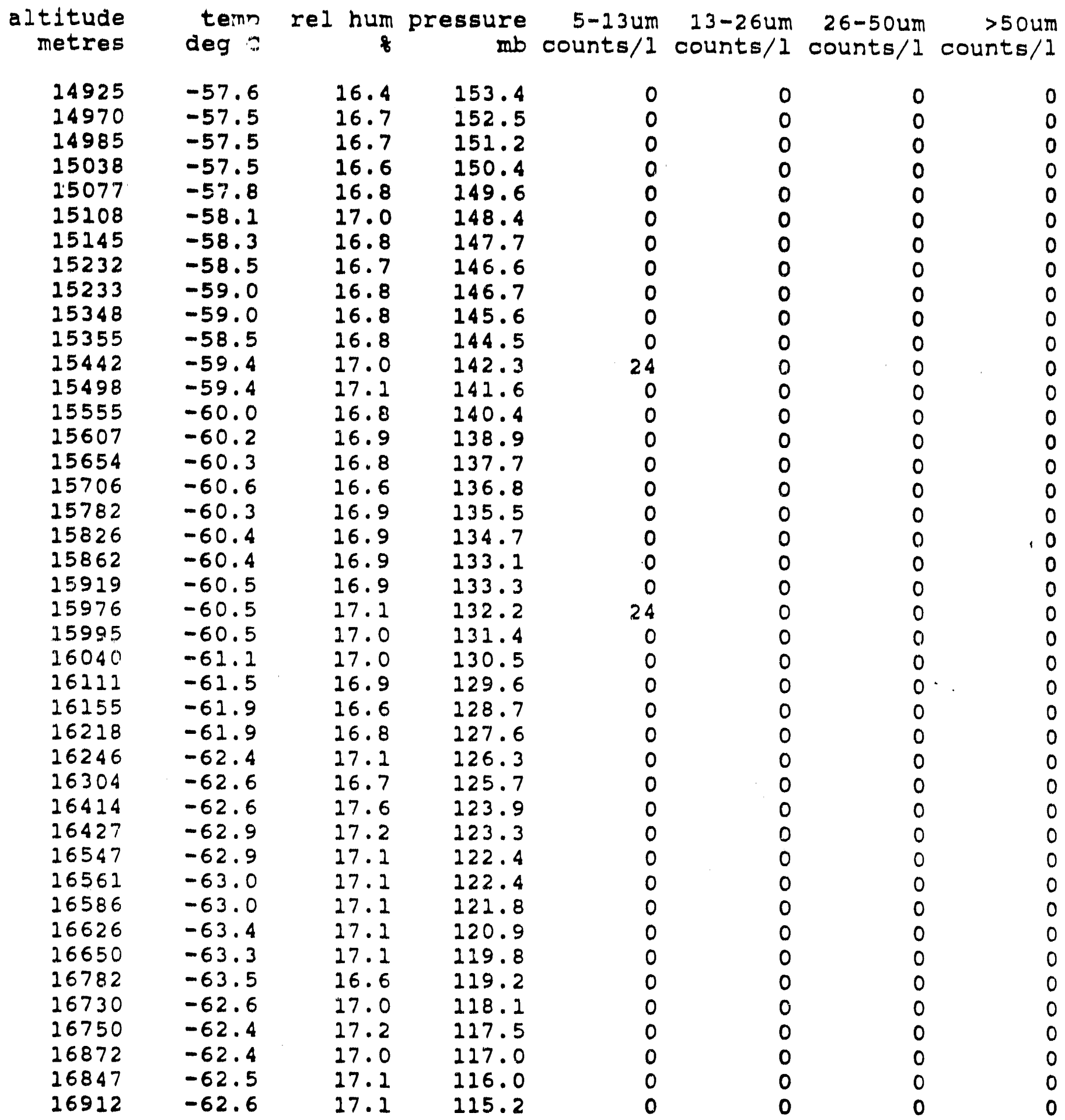


9AUG8 8 $\begin{array}{rrrrr}\text { altitude temp rel hum pressure } 5-13 \mathrm{um} 13-26 \mathrm{um} 26-50 u m & >50 u m \\ \text { metres } & \text { deg } C & \text { mb counts/1 counts/1 counts/1 counts/1 }\end{array}$

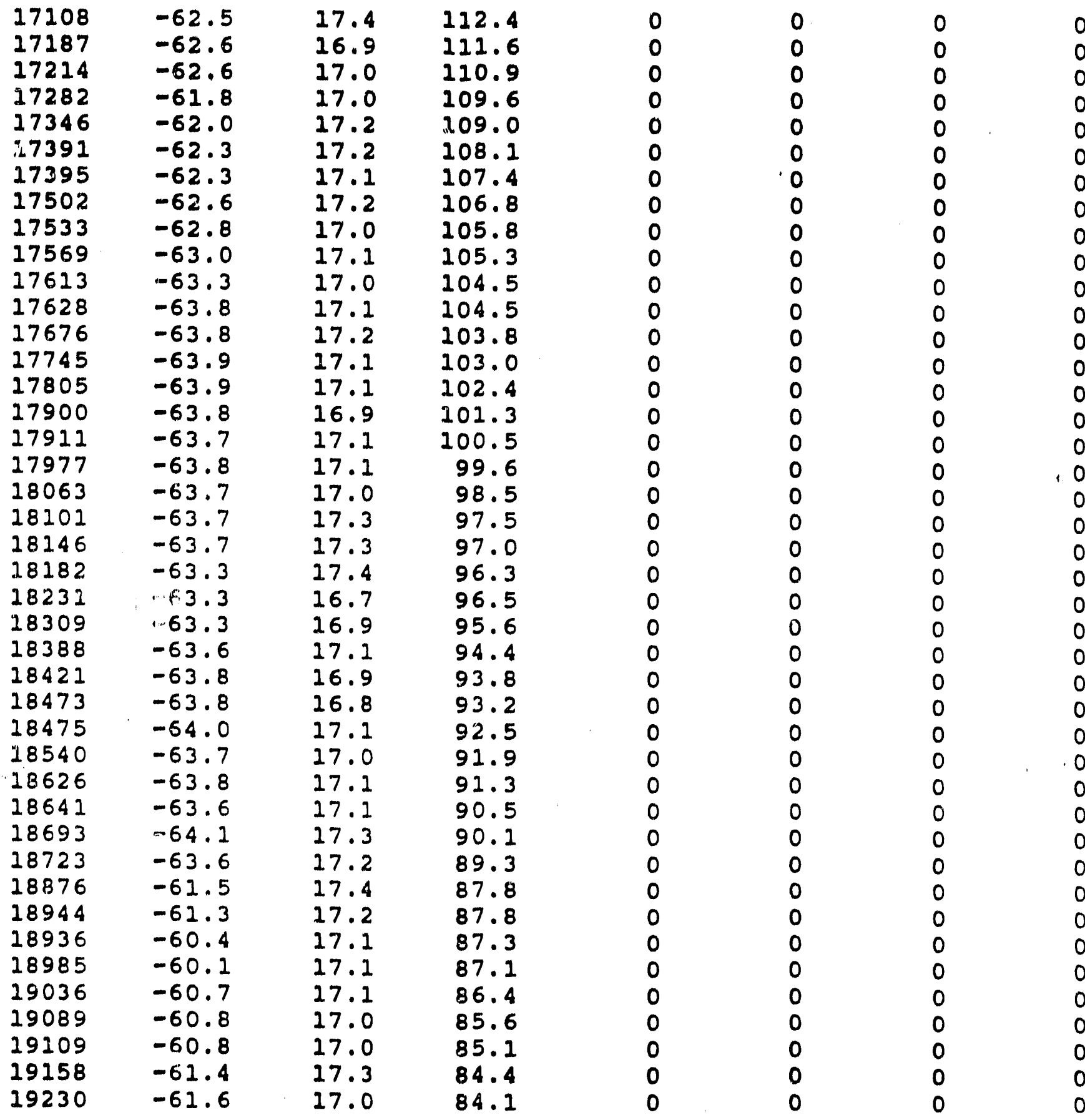


9AUG8 8

\begin{tabular}{|c|c|c|c|c|c|c|c|}
\hline $\begin{array}{l}\text { ltitude } \\
\text { metres }\end{array}$ & $\begin{array}{r}\text { temp } \\
\text { deg } C\end{array}$ & rel hum & $\begin{array}{r}\text { pressure } \\
\text { mb }\end{array}$ & $\begin{array}{r}5-13 u m \\
\text { counts/1 }\end{array}$ & $\begin{array}{c}13-26 u m \\
\text { counts/1 }\end{array}$ & $\begin{array}{c}26-50 u m \\
\text { counts } / 1\end{array}$ & $\begin{array}{r}>50 u m \\
\text { counts } / 1\end{array}$ \\
\hline $\begin{array}{l}19289 \\
19306 \\
19343 \\
19423 \\
19400 \\
19516 \\
19538 \\
19579 \\
19612 \\
19704 \\
19706 \\
19836 \\
19878 \\
19860 \\
19910 \\
19980 \\
20058 \\
20048 \\
20108 \\
20170 \\
20243 \\
20334 \\
20331 \\
20366 \\
20408 \\
20539 \\
20556 \\
20606 \\
20638 \\
20686 \\
20736 \\
20782 \\
20837 \\
20883 \\
20896 \\
20974 \\
21045 \\
21083 \\
21078 \\
21208 \\
21243 \\
21291\end{array}$ & $\begin{array}{l}-61.0 \\
-60.4 \\
-60.0 \\
-60.1 \\
-60.8 \\
-60.6 \\
-59.7 \\
-59.3 \\
-59.1 \\
-59.1 \\
-59.4 \\
-59.7 \\
-59.3 \\
-59.5 \\
-58.7 \\
-57.7 \\
-57.7 \\
-57.7 \\
-57.5 \\
-58.2 \\
-58.0 \\
-58.5 \\
-57.7 \\
-58.0 \\
-58.2 \\
-57.2 \\
-57.3 \\
-56.9 \\
-56.5 \\
-56.6 \\
-56.5 \\
-57.2 \\
-57.0 \\
-57.0 \\
-56.8 \\
-56.3 \\
-56.4 \\
-56.2 \\
-56.4 \\
-56.1 \\
-56.0 \\
-56.3\end{array}$ & $\begin{array}{l}17.1 \\
16.7 \\
14.2 \\
17.1 \\
16.9 \\
17.1 \\
17.1 \\
16.9 \\
17.1 \\
16.8 \\
17.0 \\
17.0 \\
17.2 \\
16.7 \\
16.6 \\
16.6 \\
16.7 \\
16.6 \\
16.7 \\
16.8 \\
17.0 \\
16.7 \\
16.6 \\
16.7 \\
16.7 \\
16.7 \\
16.5 \\
16.6 \\
17.0 \\
17.1 \\
16.6 \\
16.3 \\
16.5 \\
16.8 \\
16.4 \\
16.8 \\
16.6 \\
16.8 \\
16.7 \\
16.6 \\
16.6 \\
16.7\end{array}$ & $\begin{array}{l}83.3 \\
82.8 \\
84.7 \\
81.8 \\
81.2 \\
81.2 \\
80.1 \\
80.2 \\
79.8 \\
78.9 \\
78.5 \\
78.1 \\
78.1 \\
76.8 \\
76.2 \\
76.2 \\
75.8 \\
75.3 \\
74.7 \\
74.2 \\
73.7 \\
72.3 \\
72.8 \\
72.2 \\
71.7 \\
70.7 \\
70.3 \\
70.4 \\
69.7 \\
69.6 \\
68.9 \\
68.6 \\
68.2 \\
67.8 \\
67.4 \\
66.9 \\
66.6 \\
66.3 \\
65.7 \\
65.3 \\
65.1 \\
62.5\end{array}$ & $\begin{array}{l}0 \\
0 \\
0 \\
0 \\
0 \\
0 \\
0 \\
0 \\
0 \\
0 \\
0 \\
0 \\
0 \\
0 \\
0 \\
0 \\
0 \\
0 \\
0 \\
0 \\
0 \\
0 \\
0 \\
0 \\
0 \\
0 \\
0 \\
0 \\
0 \\
0 \\
0 \\
0 \\
0 \\
0 \\
0 \\
0 \\
0 \\
0 \\
0 \\
0 \\
0 \\
0\end{array}$ & $\begin{array}{l}0 \\
0 \\
0 \\
0 \\
0 \\
0 \\
0 \\
0 \\
0 \\
0 \\
0 \\
0 \\
0 \\
0 \\
0 \\
0 \\
0 \\
0 \\
0 \\
0 \\
0 \\
0 \\
0 \\
0 \\
0 \\
0 \\
0 \\
0 \\
0 \\
0 \\
0 \\
0 \\
0 \\
0 \\
0 \\
0 \\
0 \\
0 \\
0 \\
0 \\
0 \\
0\end{array}$ & $\begin{array}{l}0 \\
0 \\
0 \\
0 \\
0 \\
0 \\
0 \\
0 \\
0 \\
0 \\
0 \\
0 \\
0 \\
0 \\
0 \\
0 \\
0 \\
0 \\
0 \\
0 \\
0 \\
0 \\
0 \\
0 \\
0 \\
0 \\
0 \\
0 \\
0 \\
0 \\
0 \\
0 \\
0 \\
0 \\
0 \\
0 \\
0 \\
0 \\
0 \\
0 \\
0 \\
0\end{array}$ & $\begin{array}{l}0 \\
0 \\
0 \\
0 \\
0 \\
0 \\
0 \\
0 \\
0 \\
0 \\
0 \\
0\end{array}$ \\
\hline
\end{tabular}




\section{AUG88}

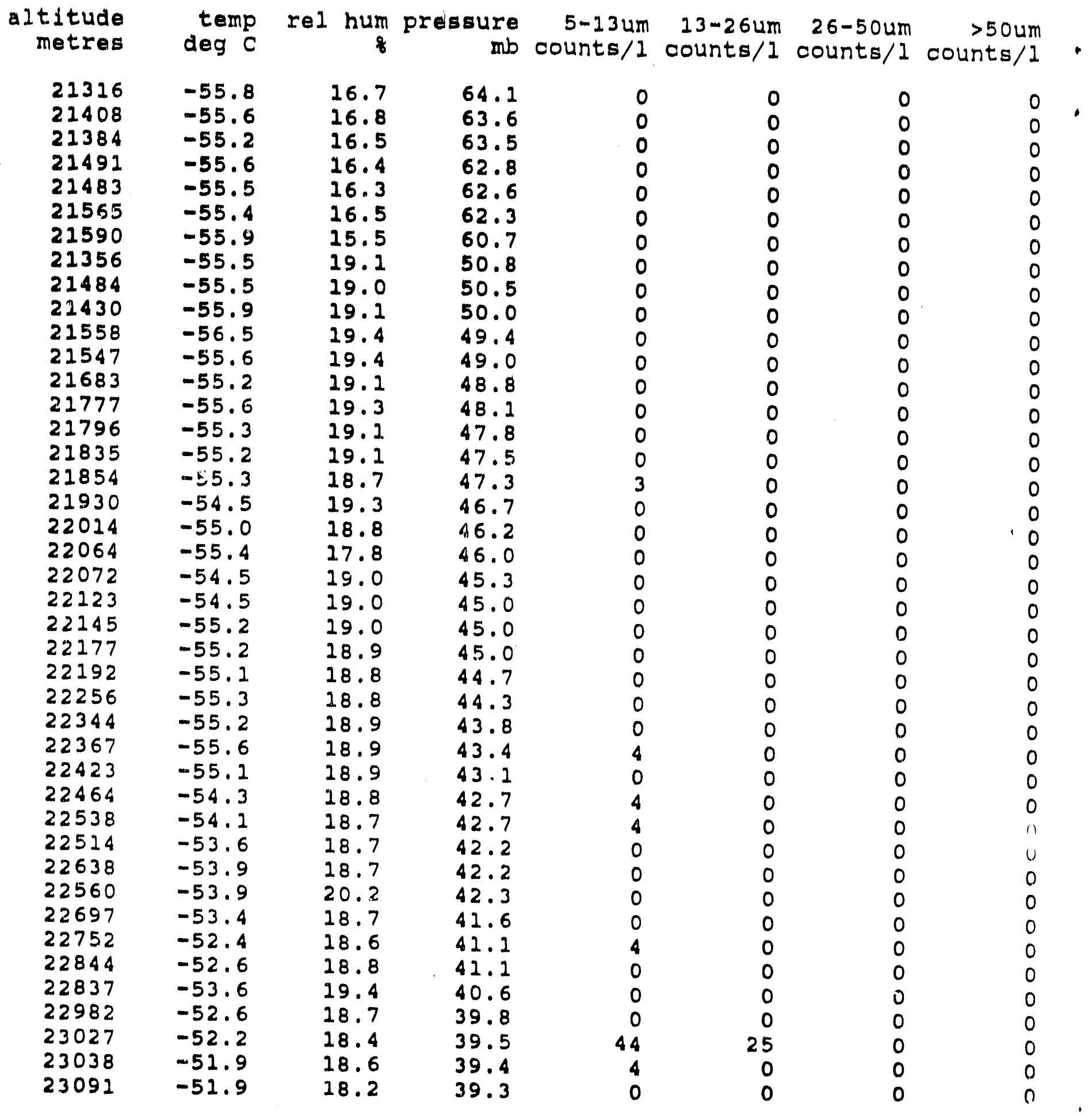




\section{AUGB 8}

altitude temp rel hum pressure 5-13um 13-26um 26-50 $\mathrm{um}>50 \mathrm{um}$ metres deg $C$ mb counts/1 counts/1 counts/1 counts/1

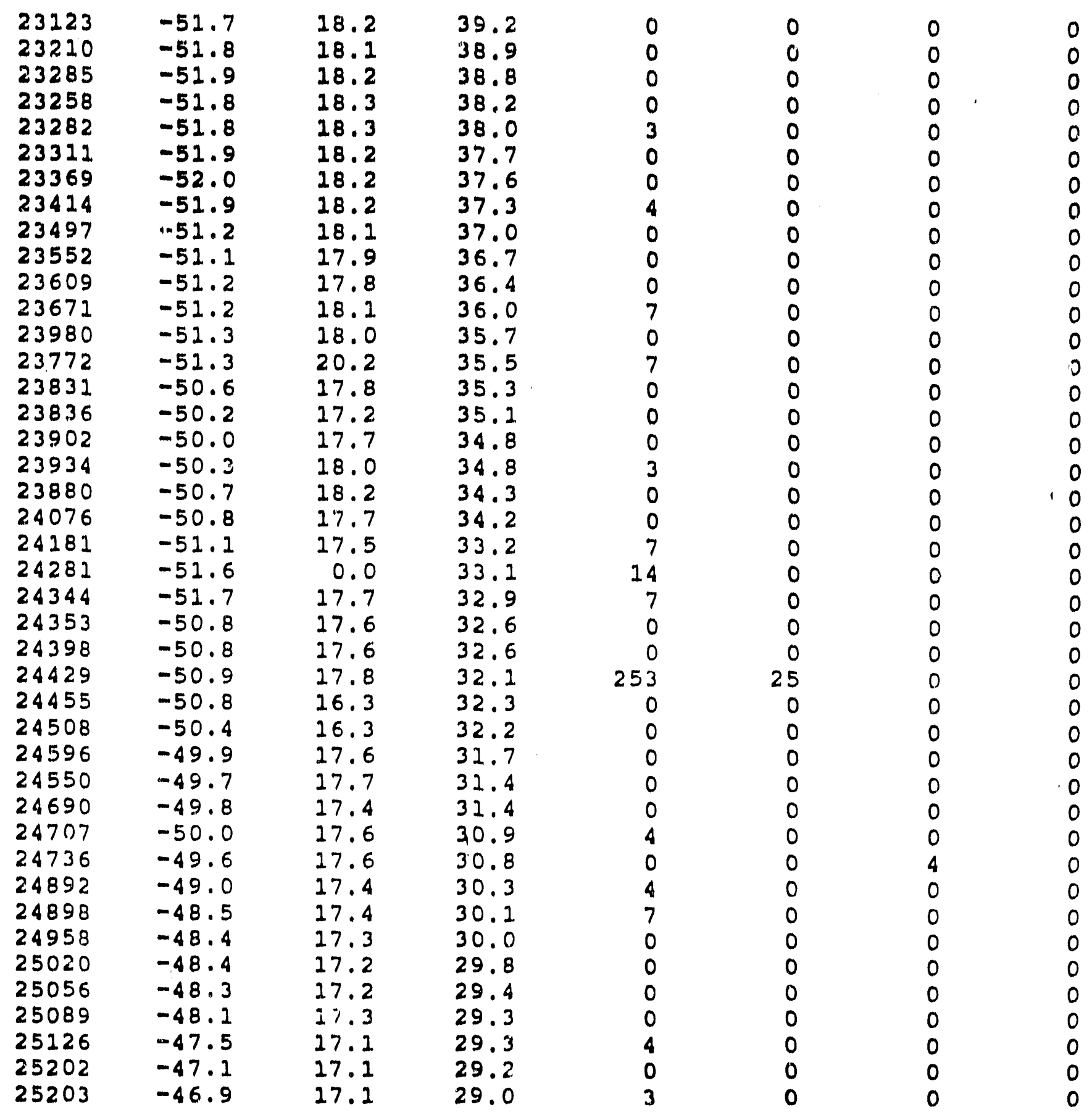




\section{AUGB 8}

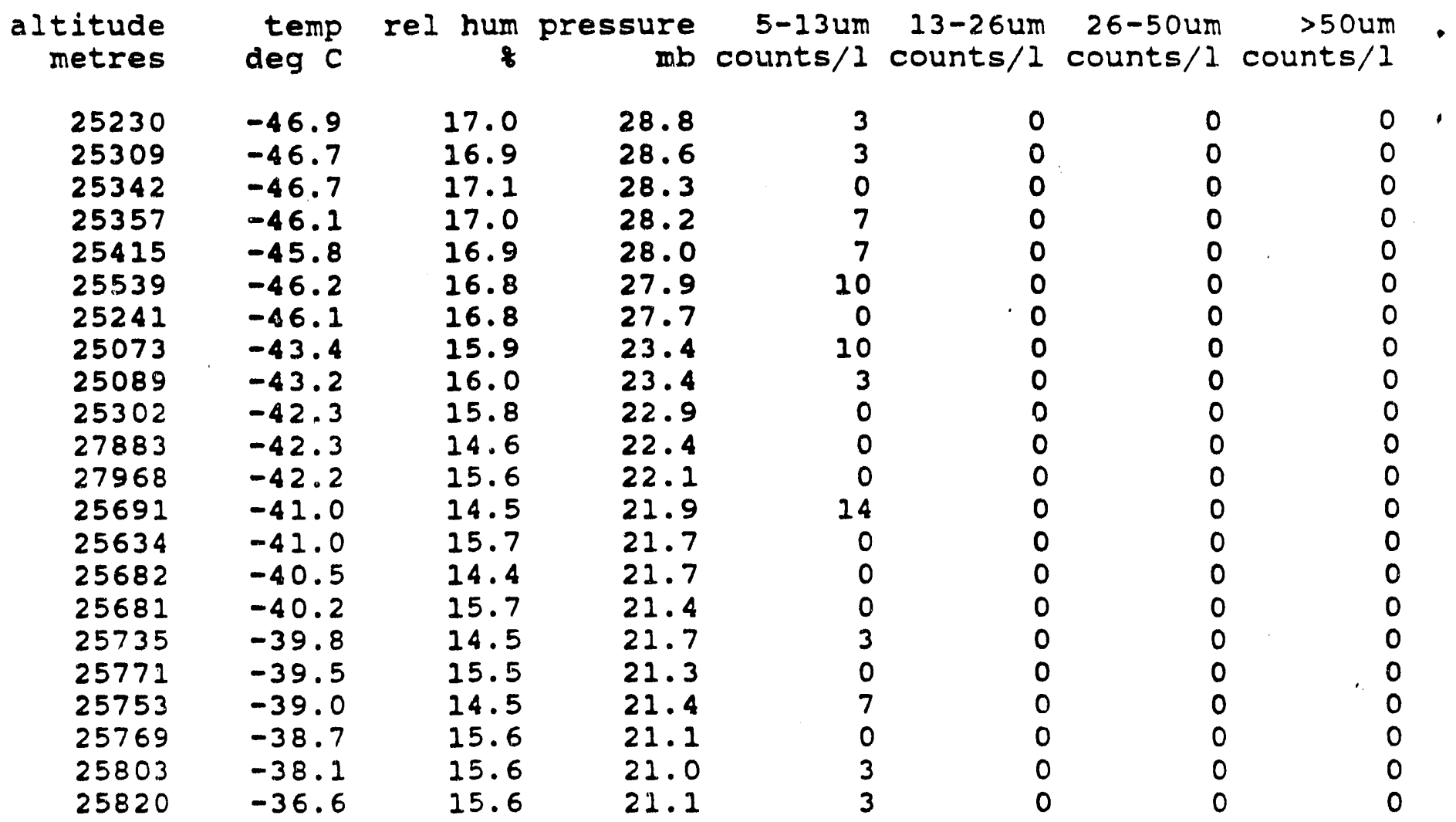


10AUG 88

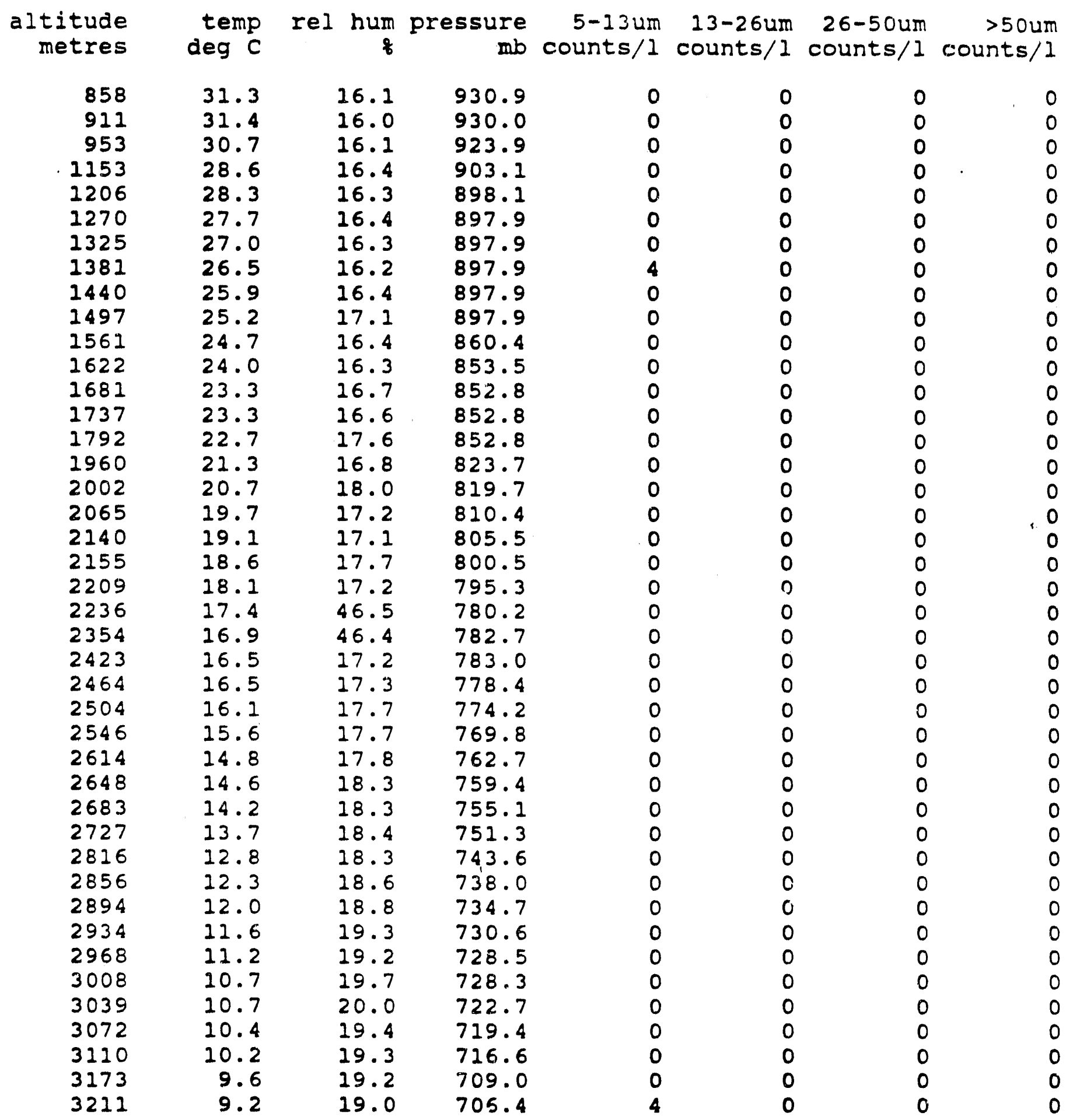




\section{AUG8 8}

$\begin{array}{rrrrr}\text { altitude temp rel hum pressure } 5-13 \mathrm{um} 13-26 \mathrm{um} & 26-50 \mathrm{um} & >50 \mathrm{um} \\ \text { metres } & \text { deg } C & \mathrm{mb} \text { counts/1 counts } / 1 \text { counts/1 counts } / 1\end{array}$

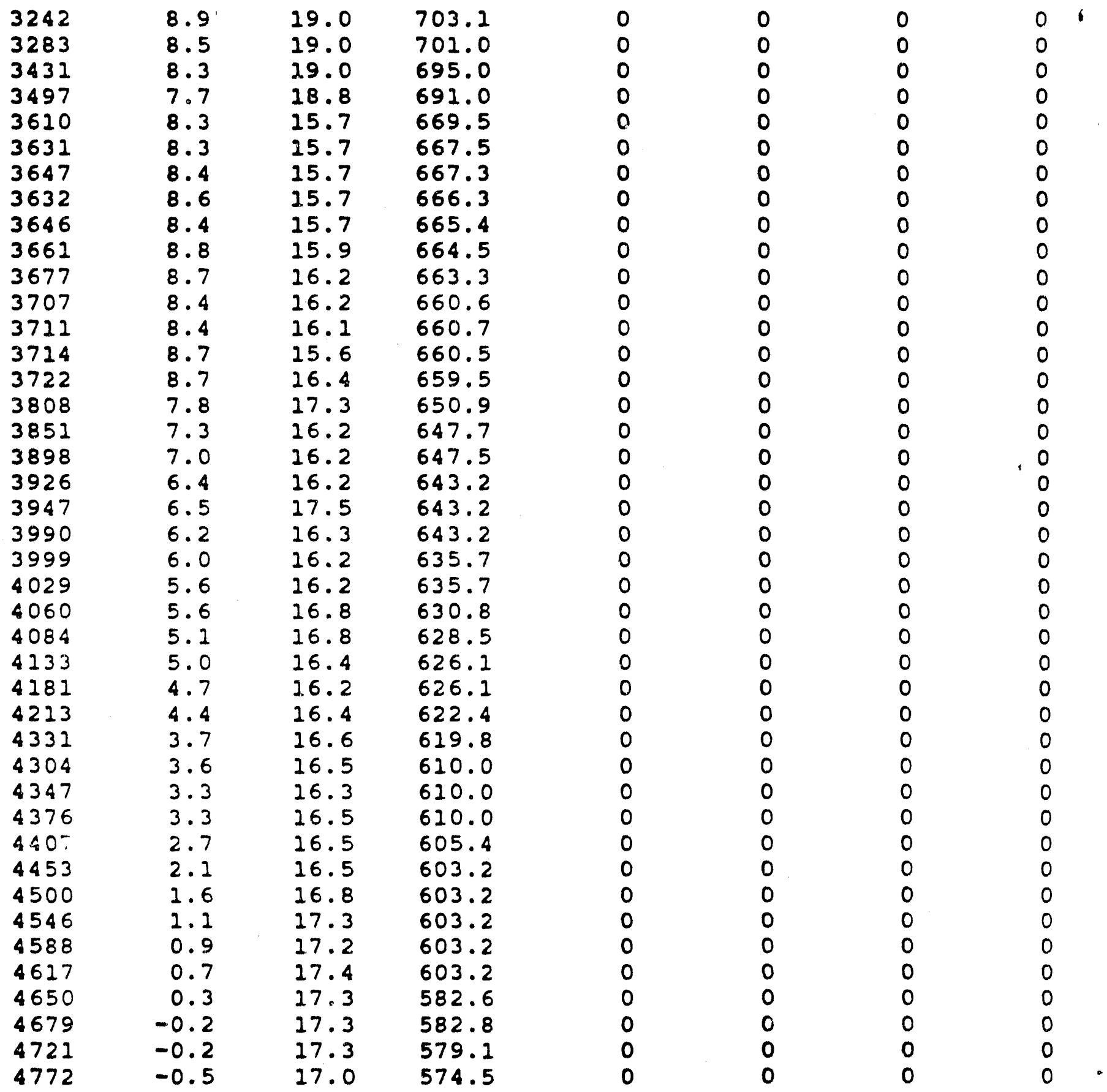


10AUG8 8

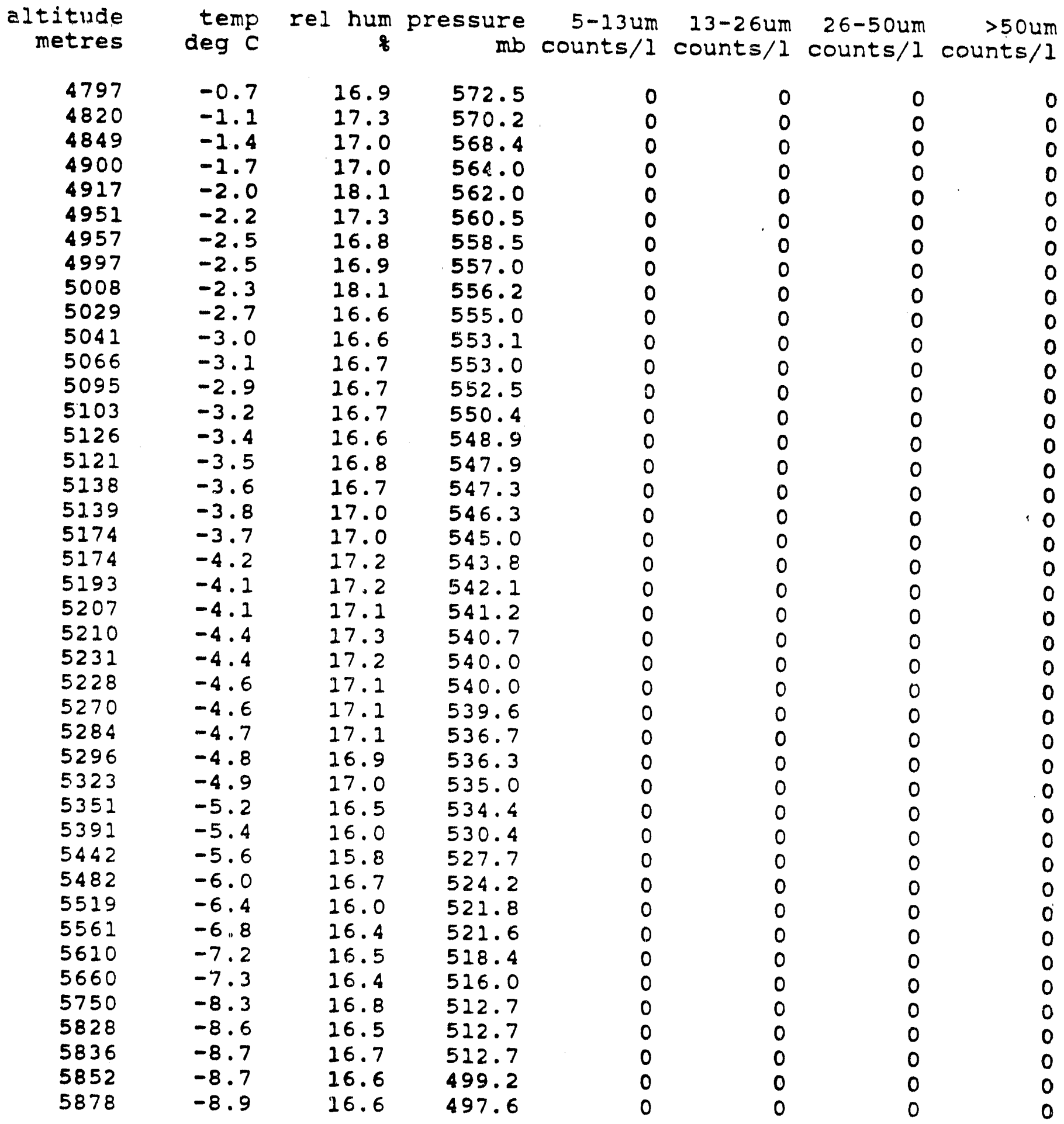




\section{OAUG 88}

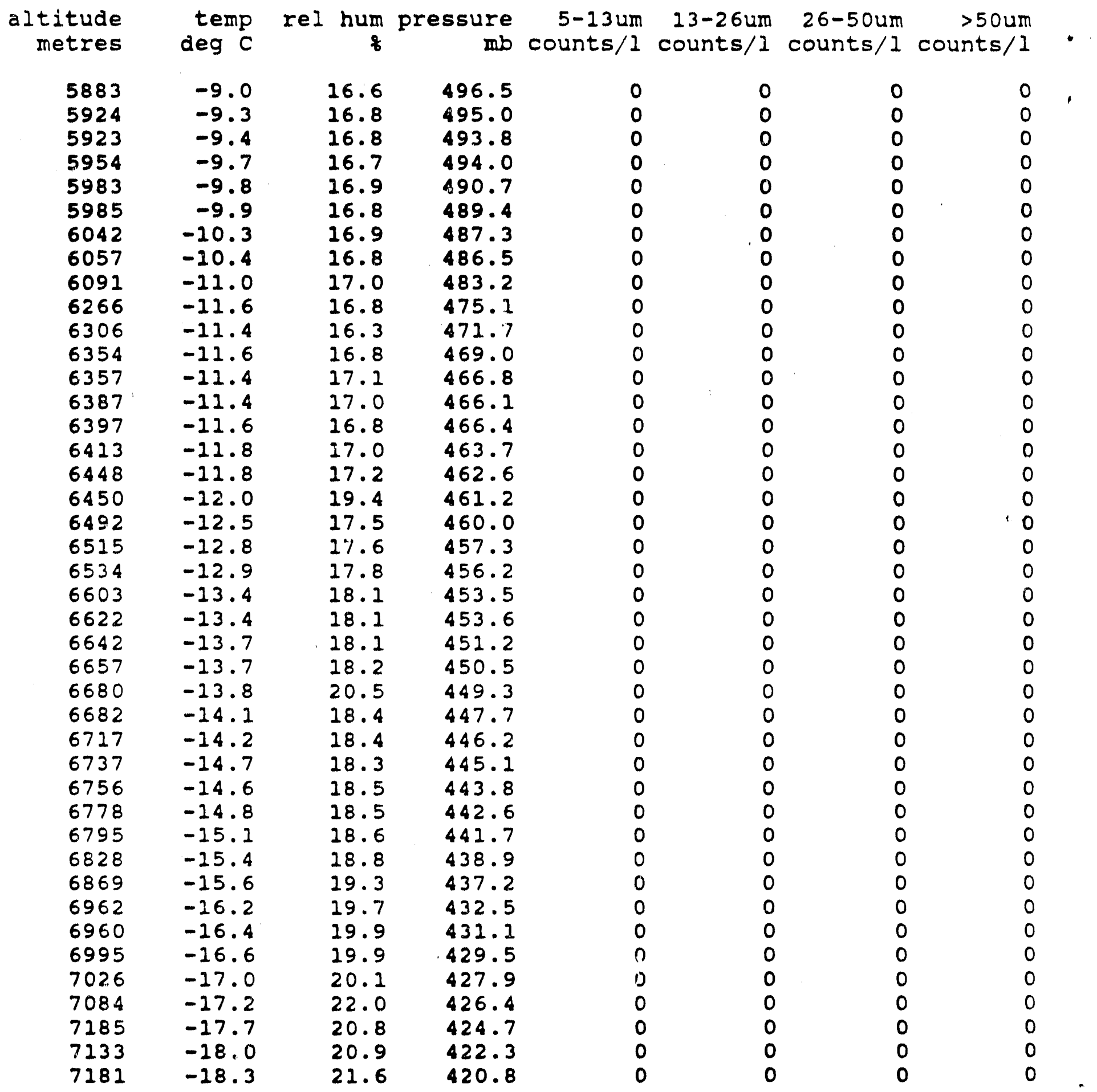




\section{AUG 88}

\begin{tabular}{|c|c|c|c|c|c|c|c|}
\hline $\begin{array}{l}\text { altitude } \\
\text { metres }\end{array}$ & $\begin{array}{r}\text { temp } \\
\text { deg } C\end{array}$ & rel hum & $\begin{array}{r}\text { pressure } \\
m b\end{array}$ & $\begin{array}{c}5-13 u m \\
\text { counts } / 1\end{array}$ & $\begin{array}{r}13-26 u m \\
\text { counts } / 1\end{array}$ & $\begin{array}{l}26-50 u m \\
\text { counts/1 }\end{array}$ & $\begin{array}{r}>50 u m \\
\text { counts } / 1\end{array}$ \\
\hline 7209 & -18.8 & 22.3 & 418.3 & 0 & 0 & 0 & 0 \\
\hline 7260 & -19.3 & 23.2 & 415.4 & 0 & 0 & 0 & 0 \\
\hline 7319 & -19.8 & 23.9 & 413.0 & 0 & 0 & 0 & 0 \\
\hline 7366 & -20.2 & 24.2 & 409.5 & 0 & 0 & 0 & 0 \\
\hline 7415 & -20.8 & 24.9 & 406.8 & 0 & 0 & 0 & 0 \\
\hline 7473 & -21.2 & 24.3 & 402.5 & 0 & 0 & 0 & 0 \\
\hline 7507 & -21.2 & 23.5 & 402.4 & 0 & 0 & 0 & 0 \\
\hline 7514 & -21.1 & 22.8 & 400.0 & 0 & 0 & 0 & 0 \\
\hline 7542 & -19.3 & 22.8 & 400.0 & 7 & 0 & 0 & 0 \\
\hline 7575 & $-19 \cdot 3$ & 22.8 & 397.8 & 0 & 0 & 0 & 0 \\
\hline 7571 & -19.3 & 22.8 & 397.9 & 0 & 0 & 0 & 0 \\
\hline 7620 & -19.3 & 999.9 & 396.6 & 0 & 0 & 0 & 0 \\
\hline 7619 & -21.9 & 999.9 & 396.2 & 0 & 0 & 0 & 0 \\
\hline 7666 & -22.2 & 18.7 & 392.6 & 0 & 0 & 0 & 0 \\
\hline 7713 & -22.2 & 17.7 & 392.1 & 0 & 0 & 0 & 0 \\
\hline 7735 & -22.6 & 18.0 & 390.3 & 0 & 0 & 0 & 0 \\
\hline 7731 & -22.3 & 17.8 & 390.0 & 0 & 0 & 0 & 0 \\
\hline 7765 & $-22 \cdot 3$ & 17.7 & 387.6 & 0 & 0 & 0 & 0 \\
\hline 7831 & -23.4 & 17.3 & 385.0 & 0 & 0 & 0 & 0 \\
\hline 7838 & -23.5 & 17.5 & 383.4 & 0 & 0 & 0 & 0 \\
\hline 7879 & -23.8 & 17.2 & 382.9 & 0 & 0 & 0 & 0 \\
\hline 7888 & -23.5 & 17.0 & 380.2 & 0 & 0 & 0 & 0 \\
\hline 7910 & -23.5 & 16.9 & 378.8 & 0 & 0 & 0 & 0 \\
\hline 7936 & -23.9 & 16.7 & 377.8 & 0 & 0 & 0 & 0 \\
\hline 7955 & -24.0 & 16.7 & 376.8 & 0 & 0 & 0 & 0 \\
\hline 7952 & -24.3 & 16.7 & 376.6 & 0 & 0 & 0 & 0 \\
\hline 8028 & -24.3 & 16.6 & 376.5 & 0 & 0 & 0 & 0 \\
\hline 8036 & -24.4 & 16.5 & 373.7 & 0 & 0 & 0 & 0 \\
\hline 8132 & -24.5 & 16.4 & 372.8 & 0 & 0 & 0 & 0 \\
\hline 8087 & -24.6 & 16.2 & 371.3 & 0 & 0 & 0 & 0 \\
\hline 8126 & -24.8 & 16.4 & 368.8 & 0 & 0 & 0 & 0 \\
\hline 8202 & -25.1 & 16.3 & 366.0 & 0 & 0 & 0 & 0 \\
\hline 8202 & -25.3 & 16.1 & 364.2 & 0 & 0 & 0 & 0 \\
\hline 8236 & -25.4 & 16.5 & 362.8 & 0 & 0 & 0 & 0 \\
\hline 8283 & -25.7 & 16.1 & 361.2 & 0 & 0 & 0 & 0 \\
\hline 8275 & -25.9 & 16.3 & 359.4 & 0 & 0 & 0 & 0 \\
\hline 8323 & -25.9 & 16.3 & 358.1 & 0 & 0 & 0 & 0 \\
\hline 8369 & -26.4 & 16.3 & 356.5 & 0 & 0 & 0 & 0 \\
\hline 8382 & -26.4 & 15.9 & 355.8 & 0 & 0 & 0 & 0 \\
\hline 8407 & -26.6 & 16.3 & 354.2 & 0 & 0 & 0 & 0 \\
\hline 8452 & -27.0 & 16.2 & 354.0 & 0 & 0 & 0 & 0 \\
\hline 8486 & -27.0 & 16.1 & 353.6 & 0 & 0 & 0 & 0 \\
\hline
\end{tabular}


20AUG 88

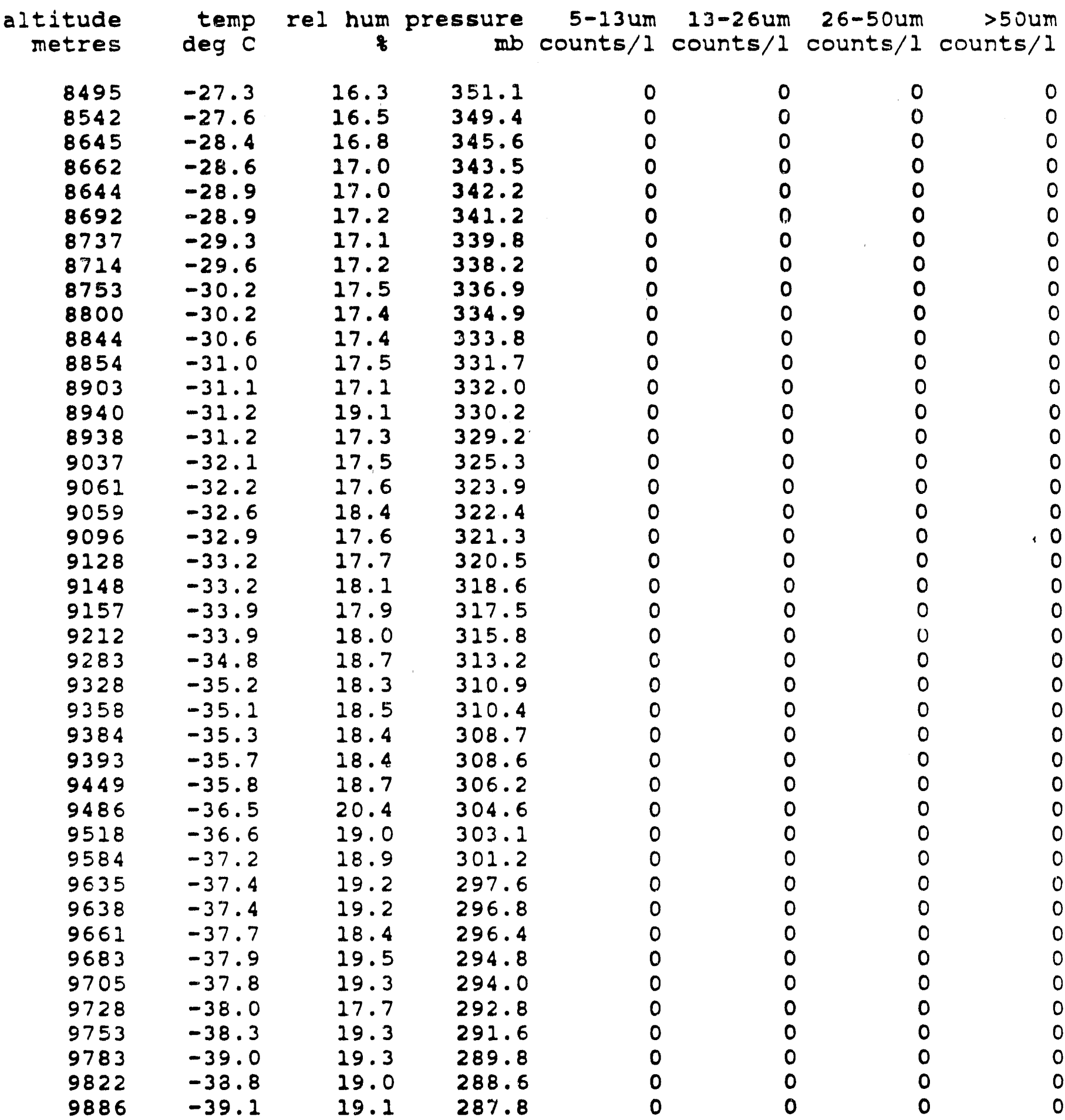




\section{AUG8 8}

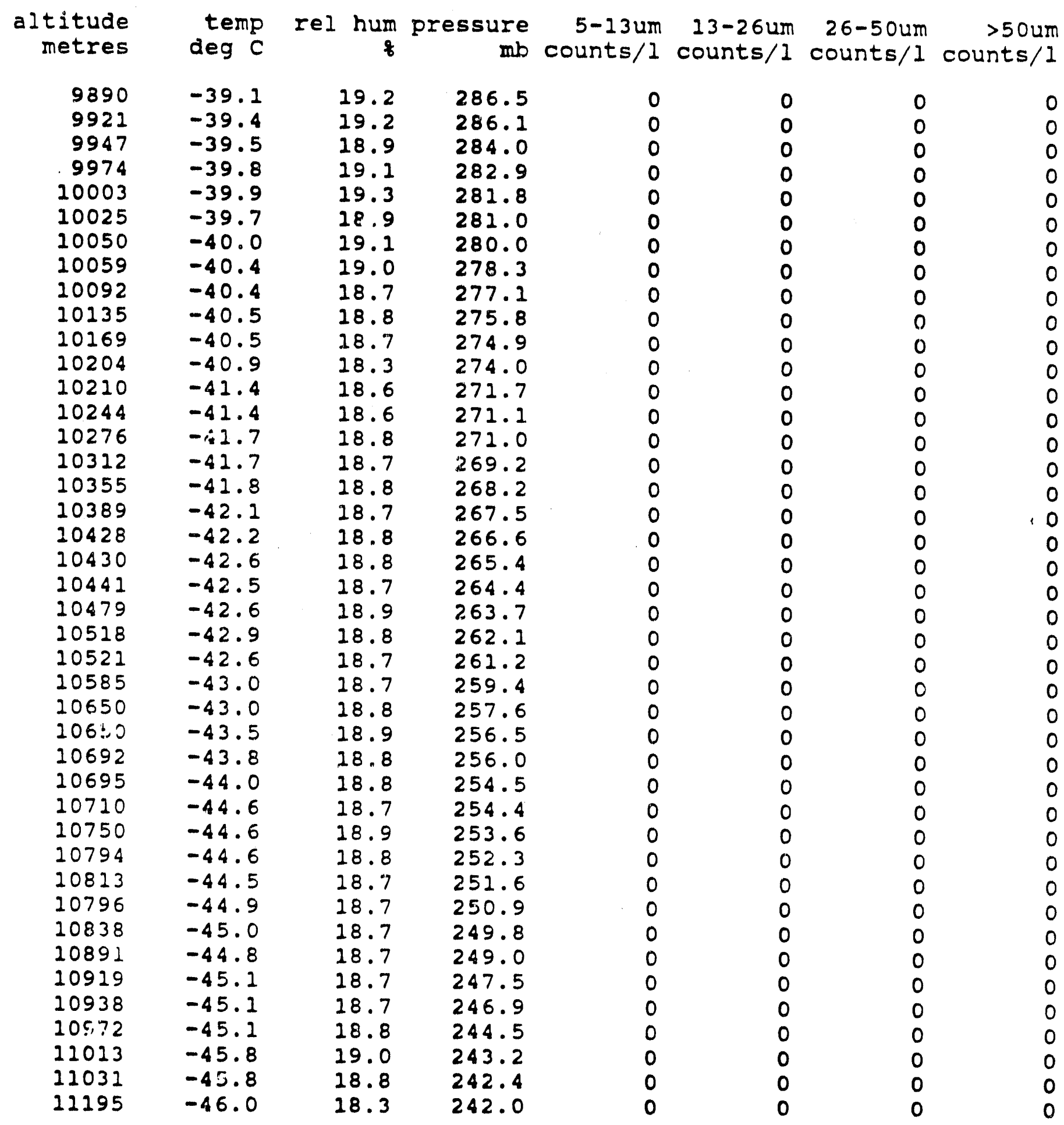




\section{AUG 88}

altitude
metres

11086

11106

11100

11138

11161

11172

11148

11189

11234

11257

11303

11342

11383

11374

11363

11403

11400

11419

11460

11498

11539

11560

11563

11550

11681

11703

11720

11756

11791

11819

11780

21867

11922

11863

11903

12032

12056

12084

12115

12072

12147

12211 temp rel hum \&

-45 .

$-45.8$

$-45.8$

$-46.4$

$-46.4$

$-46.4$

$-46.6$

$-47.1$

$-47.3$

$-47.0$

$-46.9$

$-46.9$

$-47.4$

$-47.6$

$-47.7$

$-47.8$

$-47.7$

$-47.7$

$-47.0$

$-47.9$

$-47.9$

$-47.7$

$-48.2$

$-48.2$

$-48.4$

$-47.9$

$-47.9$

$-48.4$

$-48.0$

$-48.2$

$-48.6$

$-48.3$

$-48.3$

$-48.8$

$-48.3$

$-48.7$

$-49.3$

$-48.8$

$-48.7$

$-48.7$

$-48.5$

$\begin{array}{ll}18.8 & 241.0 \\ 18.7 & 240.0 \\ 18.7 & 239.9 \\ 18.7 & 239.1 \\ 18.5 & 238.1 \\ 18.9 & 236.9 \\ 19.3 & 236.4 \\ 18.8 & 235.3 \\ 18.9 & 235.0 \\ 18.8 & 232.7 \\ 18.9 & 232.1 \\ 18.9 & 231.0 \\ 18.9 & 230.6 \\ 20.7 & 229.6 \\ 18.8 & 228.7 \\ 18.9 & 228.2 \\ 18.8 & 227.7 \\ 18.8 & 227.7 \\ 19.0 & 226.0 \\ 18.8 & 225.0 \\ 18.9 & 224.6 \\ 18.9 & 223.6 \\ 18.4 & 222.1 \\ 18.9 & 220.5 \\ 18.9 & 219.6 \\ 18.8 & 219.0 \\ 19.0 & 216.4 \\ 18.9 & 215.9 \\ 18.9 & 214.6 \\ 18.8 & 213.7 \\ 18.6 & 212.7 \\ 18.6 & 211.8 \\ 19.6 & 211.8 \\ 18.7 & 210.9 \\ 18.7 & 210.4 \\ 18.7 & 208.2 \\ 18.5 & 206.9 \\ 18.9 & 206.0 \\ 18.6 & 205.0 \\ 18.7 & 204.2 \\ 18.7 & 203.8 \\ 18.2 & 203.0 \\ & \end{array}$

5-13um

counts/1 co

counts/1

$>5$ oum counts/1 counts/1

0
0
0
0
0
0
0
0

0

0

0

$$
0
$$

0

0

0

0

0

0

0

0

0

0

0

0

0

0

0

0

0

0

0

0

0

0

0

0

0

0

0

0

0

0

0

0

$\begin{array}{ll}0 & 0 \\ 0 & 0 \\ 0 & 0 \\ 0 & 0 \\ 0 & 0 \\ 0 & 0 \\ 0 & 0 \\ 0 & 0 \\ 0 & 0 \\ 0 & 0 \\ 0 & 0 \\ 0 & 0 \\ 0 & 0 \\ 0 & 0 \\ 0 & 0 \\ 0 & 0 \\ 0 & 0 \\ 0 & 0 \\ 0 & 0 \\ 0 & 0 \\ 0 & 0 \\ 0 & 0 \\ 0 & 0 \\ 0 & 0 \\ 0 & 0 \\ 0 & 0 \\ 0 & 0 \\ 0 & 0 \\ 0 & 0 \\ 0 & 0 \\ 0 & 0 \\ 0 & 0 \\ 0 & 0 \\ 0 & 0 \\ 0 & 0 \\ 0 & 0 \\ 0 & 0 \\ 0 & 0 \\ 0 & 0 \\ 0 & 0 \\ 0 & 0 \\ 0 & 0\end{array}$




\section{AUG 88}

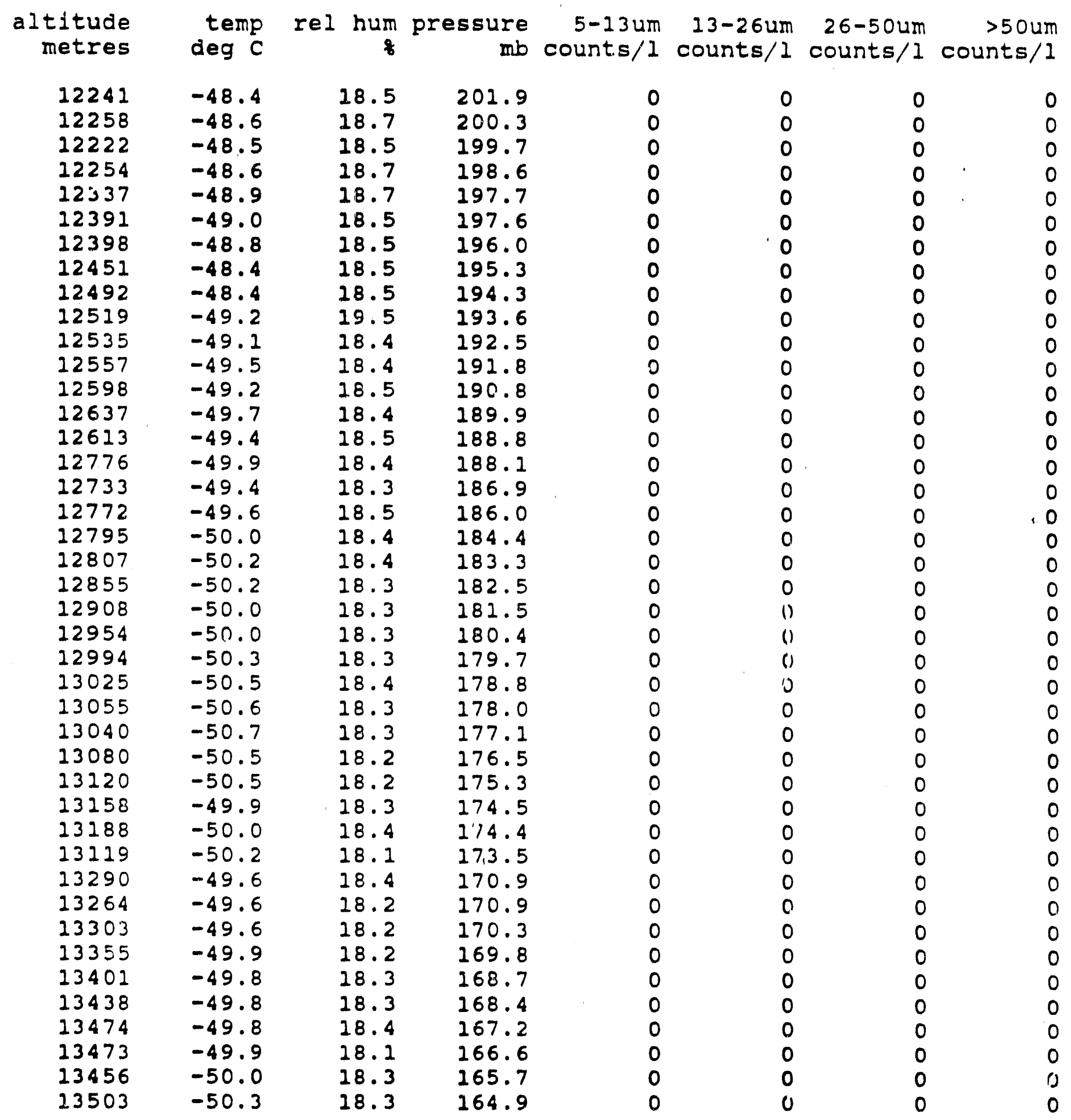


IOAUGB 8

$\begin{array}{rrrrr}\text { altitude temp rel hum pressure } 5-13 \mathrm{um} & 13-26 \mathrm{um} & 26-50 \mathrm{um} & >50 \mathrm{~m} \\ \text { metres } & \text { deg } \mathrm{C} & \mathrm{mb} \text { counts } / 1 \text { counts } / 1 \text { counts } / 1 \text { counts } / 1\end{array}$

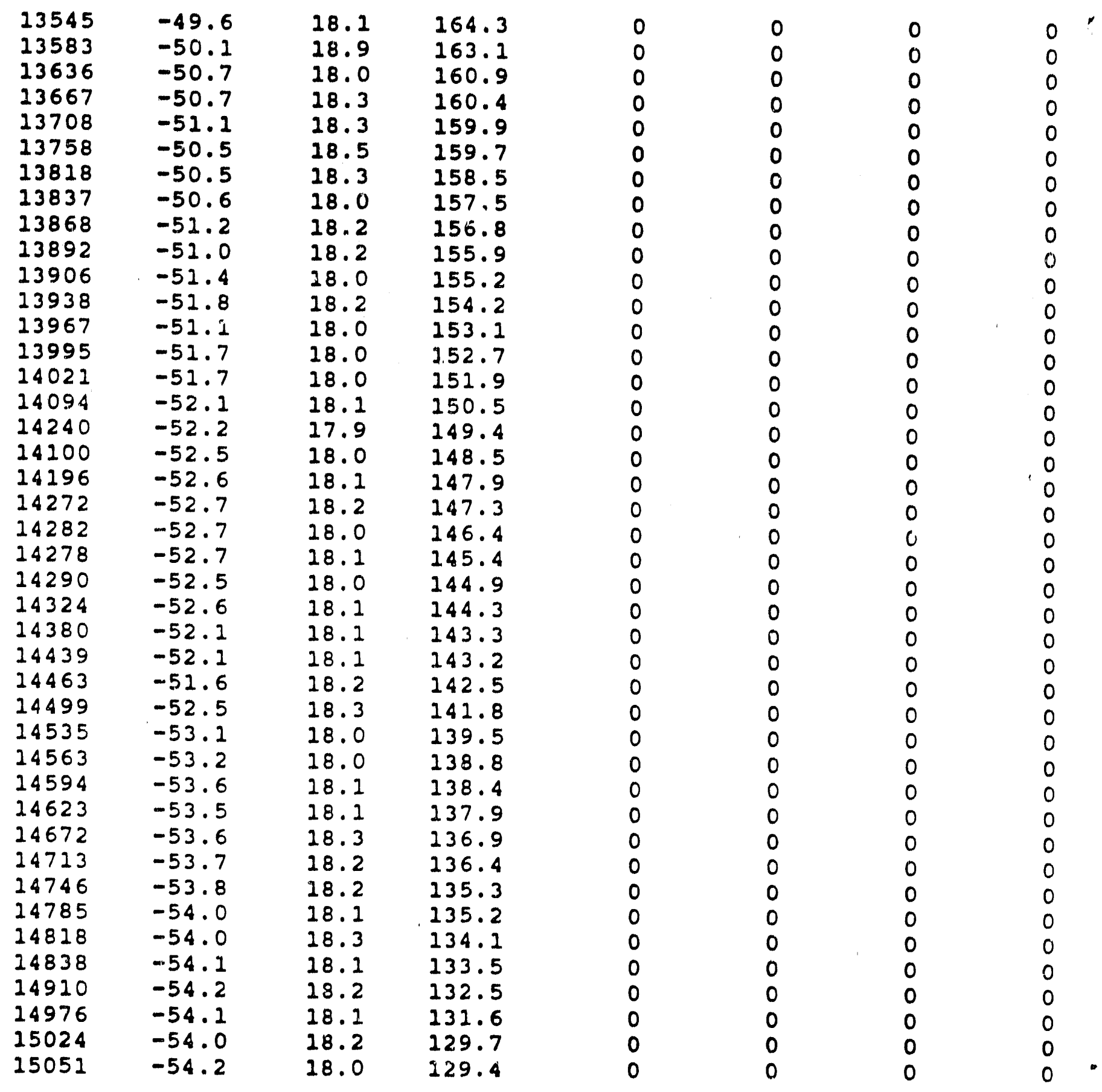


10AUG8 8

altitude temp rel hum pressure 5-13um 13-26um 26-50um >50um metres deg $C$ mb counts/1 counts $/ 1$ counts $/ 1$ counts $/ 1$

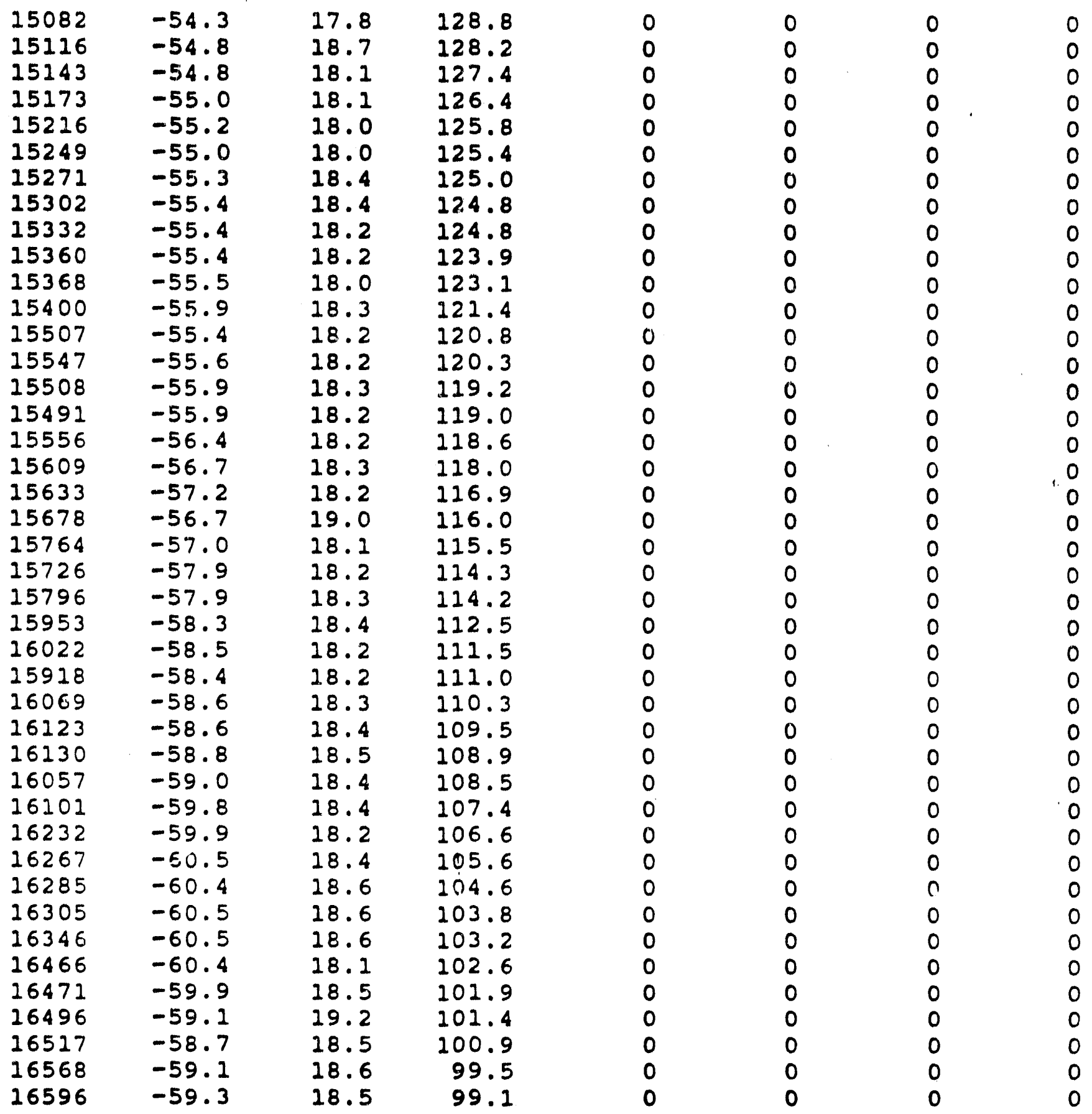


10AUG8 8

$\begin{array}{rrrrr}\text { altitude } & \text { temp rel hum pressure } 5-13 \mathrm{um} 13-26 \mathrm{um} 26-50 u m & >50 u m & \text {. } \\ \text { metres } & \text { deg } C & \text { mb counts/1 counts/1 counts/1 counts/1. }\end{array}$

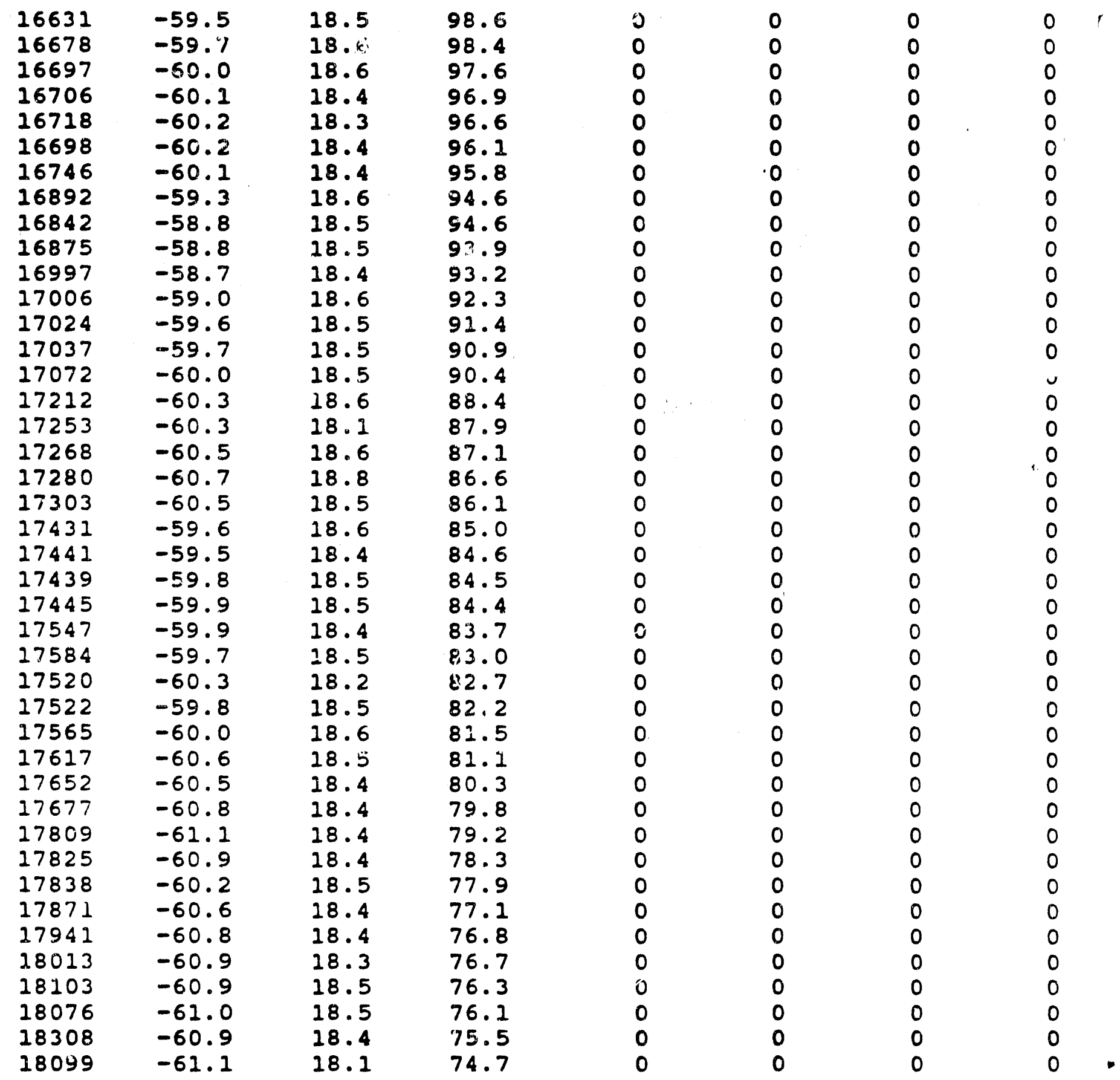




\section{AUG88}

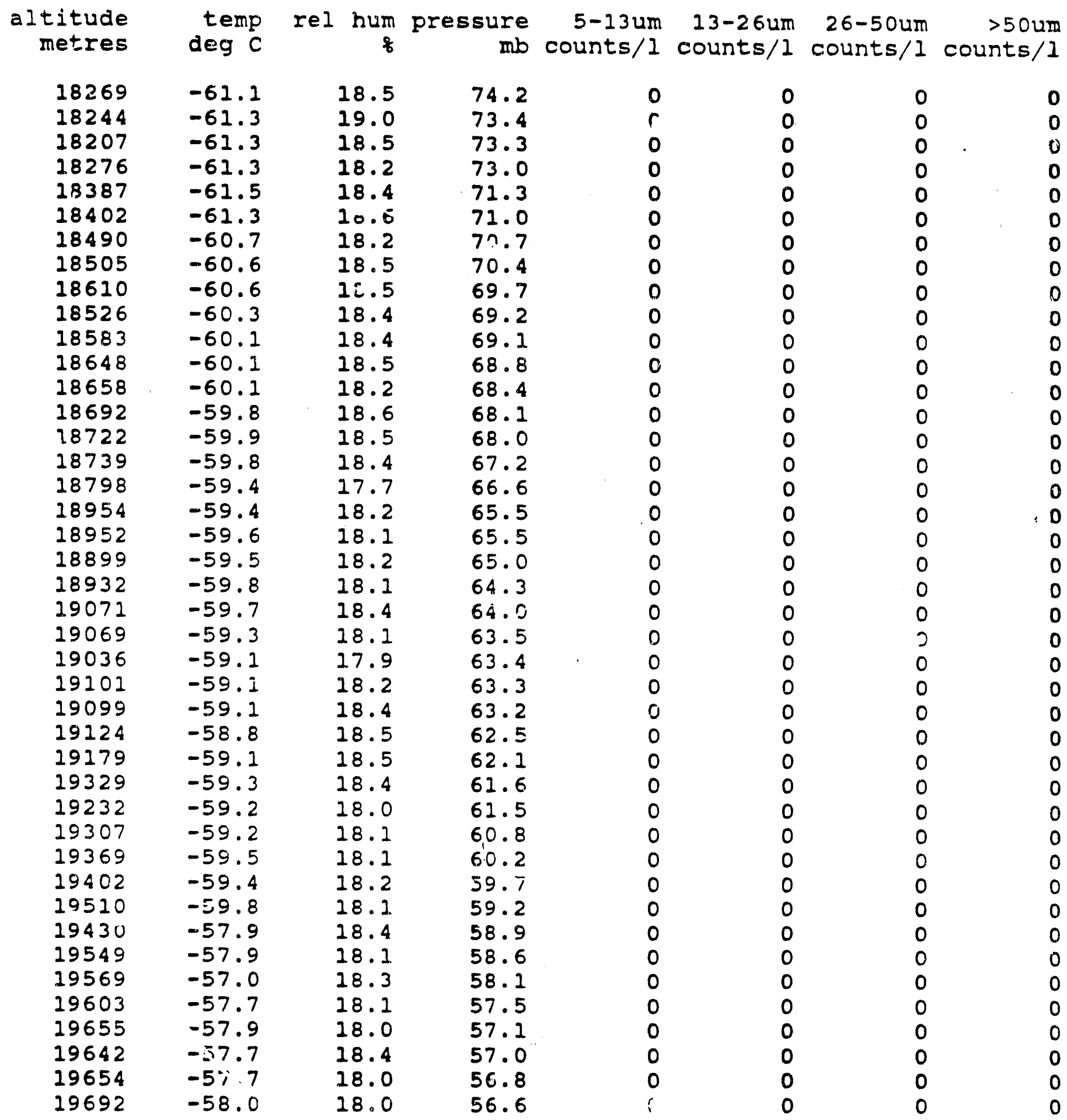


15AUG 88

altitude metres

$\begin{array}{llll}893 & 28.4 & 23.7 & 929.2 \\ 919 & 26.8 & 24.6 & 922.2 \\ 1025 & 25.3 & 26.2 & 905.0 \\ 1078 & 24.4 & 27.2 & 895.9 \\ 1153 & 23.4 & 28.7 & 886.4 \\ 1192 & 22.8 & 29.9 & 878.2 \\ 1436 & 20.1 & 33.7 & 848.8 \\ 1435 & 20.1 & 32.4 & 850.3 \\ 1663 & 19.4 & 34.1 & 844.8 \\ 1700 & 18.7 & 34.8 & 839.3 \\ 1752 & 18.5 & 35.8 & 833.1 \\ 1836 & 18.2 & 36.1 & 828.1 \\ 1979 & 16.3 & 38.2 & 828.1 \\ 2030 & 15.5 & 37.4 & 828.1 \\ 2096 & 14.8 & 40.3 & 828.1 \\ 2149 & 14.6 & 41.6 & 793.8 \\ 2195 & 14.2 & 41.6 & 789.3 \\ 2248 & 14.3 & 32.2 & 784.7 \\ 2284 & 14.8 & 20.9 & 785.1 \\ 2341 & 14.8 & 19.1 & 785.1 \\ 2423 & 14.8 & 18.2 & 785.1 \\ 2404 & 14.7 & 17.7 & 785.1 \\ 2426 & 14.9 & 17.4 & 785.1 \\ 2486 & 14.9 & 17.3 & 759.8 \\ 2528 & 15.2 & 17.1 & 755.1 \\ 2655 & 14.7 & 16.8 & 751.0 \\ 2709 & 14.6 & 16.7 & 747.1 \\ 2769 & 14.4 & 16.7 & 747.1 \\ 2847 & 14.5 & 16.4 & 747.1 \\ 2923 & 14.2 & 17.1 & 747.1 \\ 2907 & 14.0 & 16.7 & 747.1 \\ 2991 & 13.9 & 16.5 & 717.3 \\ 3049 & 13.9 & 16.3 & 713.1 \\ 3071 & 13.3 & 16.4 & 713.1 \\ 3122 & 12.5 & 16.6 & 713.1 \\ 3160 & 12.7 & 16.4 & 713.1 \\ 3206 & 12.9 & 16.4 & 713.1 \\ 3320 & 11.6 & 16.2 & 688.6 \\ 3353 & 11.2 & 16.1 & 684.1 \\ 3397 & 11.3 & 16.0 & 679.8 \\ 3414 & 11.0 & 16.0 & 680.0 \\ 3476 & 10.3 & 16.0 & 680.0\end{array}$

temp deg $C$ rel hum pressure \&

5-13um counts/1 $13-26 u m$
counts $/ 1$

0.0

0.0

0.0

0.0

0.0

0.0

3.6

3.6

0.0

0.0

0.0

3.5

0.0

7.3

10.7

10.8

3.6

0.0

0.0

0.0

0.0

0.0

0.0

0.0

0.0

0.2

0.0

0.0

0.0

0.0

0.0

0.0

0.0

0.0

0.0

0.0

0.0

0.0

0.0

0.0

0.0

0.0
0.0

0.0

0.0

0.0

0.0

0.0

0.0

0.0

0.0

0.0

0.0

0.0

0.0

0.0

0.0

0.0

0.0

0.0

0.0

0.0

0.0

0.0

0.0

0.0

0.0

0.0

0.0

0.0

0.0

0.0

0.0

0.0

0.0

0.0

0.0

0.0

0.0

0.0

0.0

0.0

0.0

0.0 26-50um counts/1 counts/1

50um
0.0

0.0

0.0

0.0

0.0

0.0

0.0

0.0

0.0

0.0

0.0

0.0

0.0

0.0

0.0

0.0

0.0

0.0

0.0

0.0

0.0

0.0

0.0

0.0

0.0

0.0

0.0

0.0

0.0

0.0

0.0

0.0

0.0

0.0

0.0

0.0

0.0

0.0

0.0

0.0

0.0

0.0
0.0

0.0

0.0

0.0

0.0

0.0

0.0

0.0

0.0

0.0

0.0

0.0

0.0

0.0

0.0

0.0

0.0

0.0

0.0

0.0

0.0

0.0

0.0

0.0

0.0

0.0

0.0

0.0

0.0

0.0

0.0

0.0

0.0

0.0

0.0

0.0

0.0

0.0

0.0

0.0

0.0

0.0 
15AUG8 8

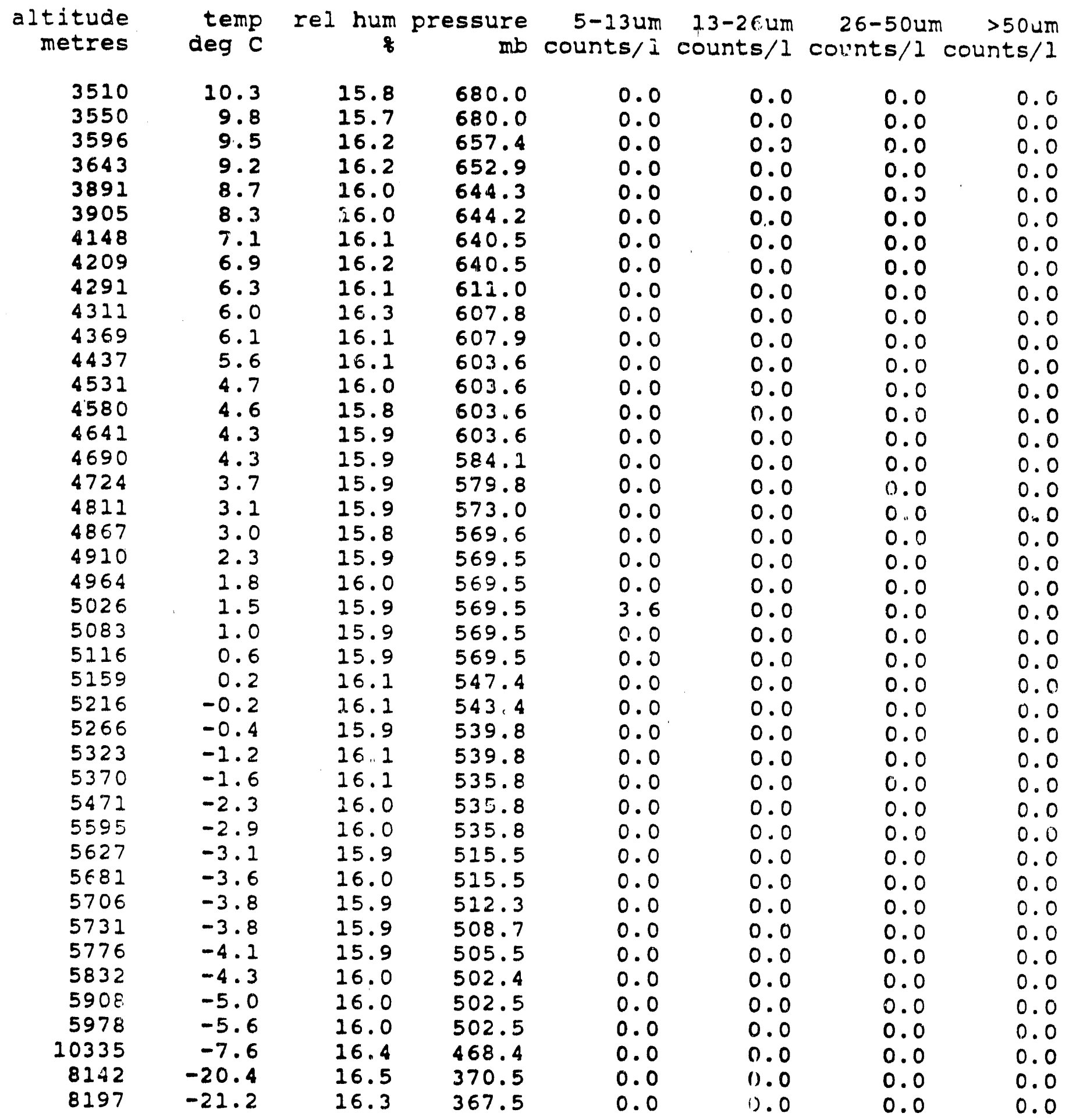




\section{AUG88}

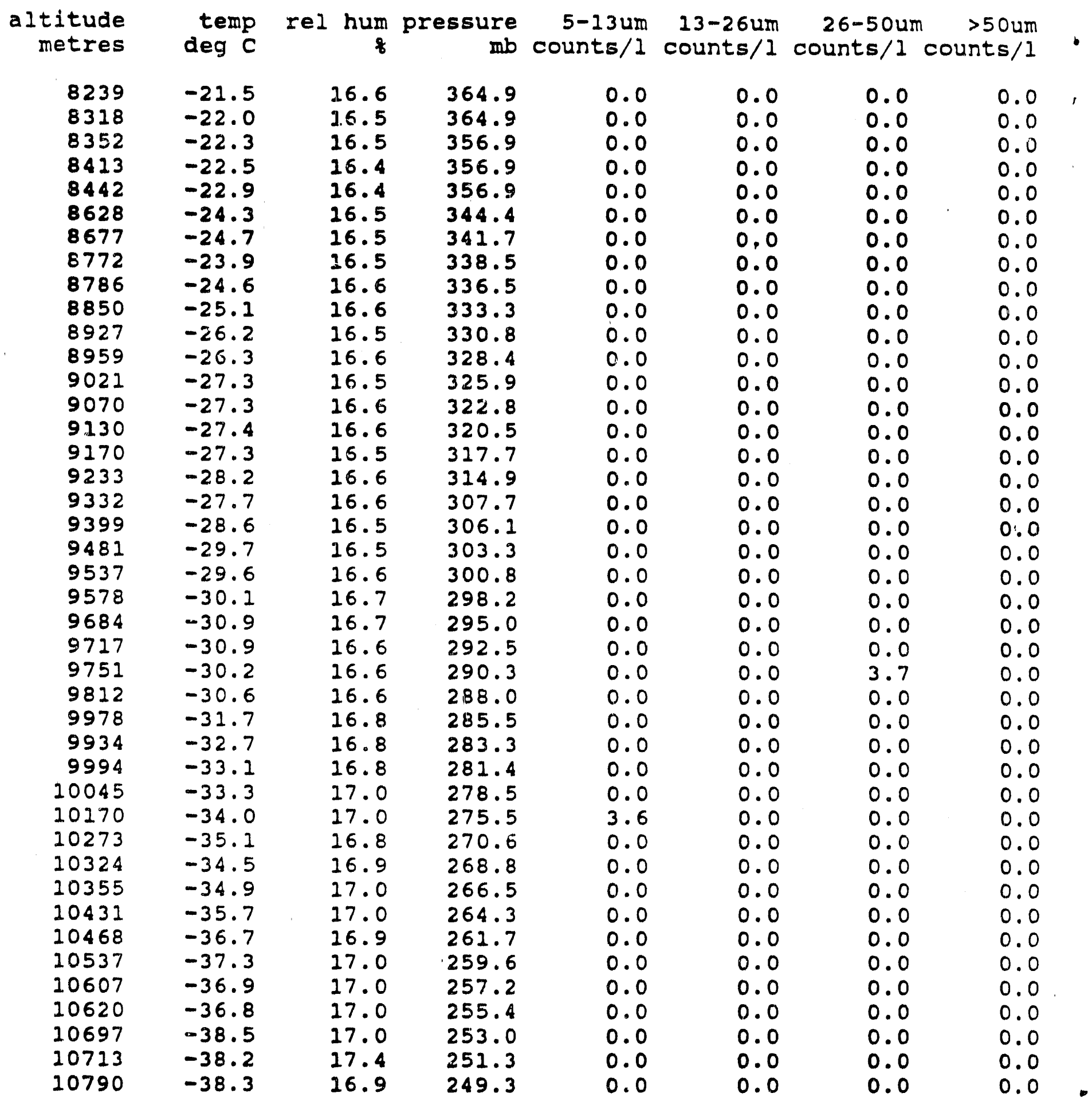




\section{AUG $8 B$}

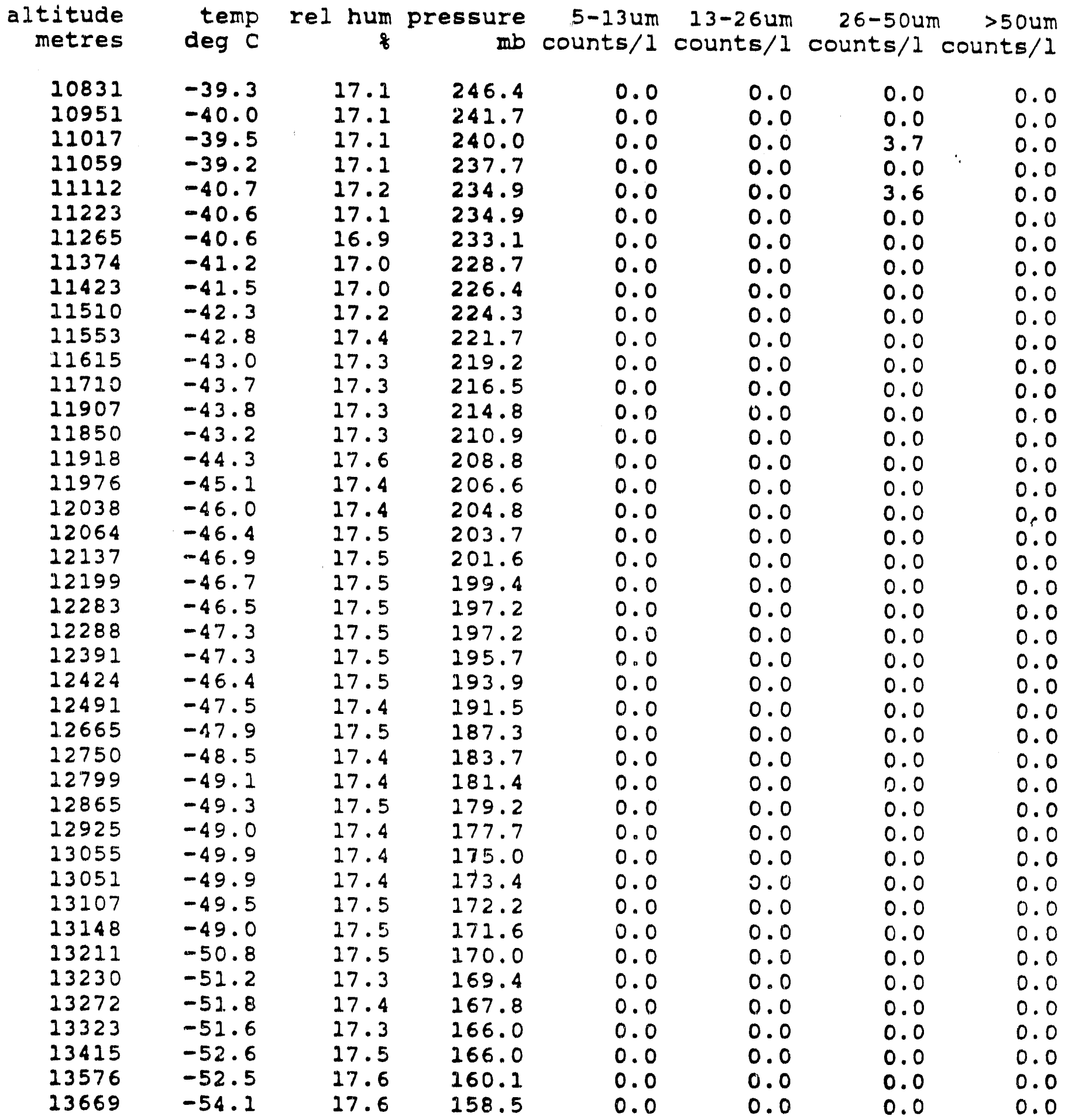


15AUG8 8

\begin{tabular}{|c|c|c|c|c|c|c|c|}
\hline $\begin{array}{r}\text { altitude } \\
\text { metres }\end{array}$ & $\begin{array}{l}\text { temp } \\
\text { deg C }\end{array}$ & $\begin{array}{r}\text { rel hum } \\
\text { f }\end{array}$ & $\begin{array}{r}\text { pressure } \\
\text { mb }\end{array}$ & $\begin{array}{c}5-13 \mathrm{um} \\
\text { counts } / 1\end{array}$ & $\begin{array}{c}13-26 u m \\
\text { counts } / 1\end{array}$ & $\begin{array}{c}26-50 u m \\
\text { counts / I }\end{array}$ & $\begin{array}{l}\text { n }>50 u m \\
\text { counts } / 1 .\end{array}$ \\
\hline $\begin{array}{l}13695 \\
13782 \\
13829 \\
13937 \\
13929 \\
14066 \\
14080 \\
14150 \\
14184 \\
14239 \\
14260 \\
14490 \\
14634 \\
14533 \\
14535 \\
14579 \\
14567 \\
14644 \\
14643 \\
14775 \\
14734 \\
14884 \\
14878 \\
14934 \\
14991 \\
14993 \\
15069 \\
15108 \\
15195 \\
15304 \\
15332 \\
15407 \\
15556 \\
15589 \\
15603 \\
15746 \\
15970 \\
15908 \\
15918 \\
16008 \\
16050 \\
16109\end{array}$ & $\begin{array}{l}-54.3 \\
-55.4 \\
-55.9 \\
-56.6 \\
-56.8 \\
-57.3 \\
-57.4 \\
-57.7 \\
-57.8 \\
-57.5 \\
-57.5 \\
-59.4 \\
-59.3 \\
-60.1 \\
-59.8 \\
-58.9 \\
-59.7 \\
-59.8 \\
-60.4 \\
-60.9 \\
-60.5 \\
-61.0 \\
-60.5 \\
-60.0 \\
-59.3 \\
-59.3 \\
-59.3 \\
-59.3 \\
-62.6 \\
-62.2 \\
-62.4 \\
-62.5 \\
-62.9 \\
-64.0 \\
-64.0 \\
-64.2 \\
-63.2 \\
-64.0 \\
-63.1 \\
-62.9 \\
-63.3 \\
-63.9\end{array}$ & $\begin{array}{l}17.7 \\
17.6 \\
17.7 \\
17.5 \\
17.7 \\
18.1 \\
18.2 \\
18.1 \\
17.8 \\
17.9 \\
17.9 \\
18.1 \\
17.9 \\
18.0 \\
17.9 \\
18.1 \\
18.0 \\
18.5 \\
17.7 \\
18.2 \\
18.2 \\
17.9 \\
18.2 \\
18.3 \\
18.2 \\
18.1 \\
18.1 \\
18.1 \\
18.1 \\
18.4 \\
18.4 \\
18.2 \\
18.4 \\
18.4 \\
18.5 \\
18.4 \\
18.5 \\
18.4 \\
18.6 \\
18.7 \\
18.5 \\
18.4\end{array}$ & $\begin{array}{l}156.7 \\
154.9 \\
153.2 \\
151.5 \\
149.5 \\
147.8 \\
145.9 \\
144.5 \\
143.0 \\
142.2 \\
141.7 \\
139.4 \\
137.6 \\
136.8 \\
135.7 \\
135.2 \\
134.1 \\
133.4 \\
132.7 \\
131.7 \\
130.3 \\
128.9 \\
127.3 \\
126.3 \\
124.9 \\
124.1 \\
123.0 \\
122.0 \\
120.7 \\
119.3 \\
117.7 \\
115.9 \\
114.0 \\
112.4 \\
110.8 \\
109.4 \\
107.7 \\
105.3 \\
104.6 \\
103.0 \\
102.4 \\
101.3\end{array}$ & $\begin{array}{l}0.0 \\
0.0 \\
0.0 \\
0.0 \\
0.0 \\
0.0 \\
0.0 \\
0.0 \\
0.0 \\
0.0 \\
0.0 \\
0.0 \\
0.0 \\
0.0 \\
0.0 \\
0.0 \\
0.0 \\
0.0 \\
0.0 \\
0.0 \\
0.0 \\
0.0 \\
0.0 \\
0.0 \\
0.0 \\
0.0 \\
0.0 \\
0.0 \\
0.0 \\
0.0 \\
0.0 \\
0.0 \\
0.0 \\
0.0 \\
0.0 \\
0.0 \\
0.1 \\
0.0 \\
0.0 \\
0.0 \\
0.0 \\
0.0\end{array}$ & $\begin{array}{l}0.0 \\
0.0 \\
0.0 \\
0.0 \\
0.0 \\
0.0 \\
0.0 \\
0.0 \\
0.0 \\
0.0 \\
0.0 \\
0.0 \\
0.0 \\
0.0 \\
0.0 \\
0.0 \\
0.0 \\
0.0 \\
0.0 \\
0.0 \\
0.0 \\
0.0 \\
0.0 \\
0.0 \\
11.0 \\
11.0 \\
11.0 \\
0.0 \\
0.0 \\
0.0 \\
0.0 \\
0.0 \\
0.0 \\
0.0 \\
0.0 \\
0.0 \\
0.0 \\
0.0 \\
0.0 \\
0.0 \\
0.0 \\
0.0\end{array}$ & $\begin{array}{l}0.0 \\
0.0 \\
0.0 \\
0.0 \\
0.0 \\
0.0 \\
0.0 \\
0.0 \\
0.0 \\
0.0 \\
0.0 \\
0.0 \\
0.0 \\
0.0 \\
0.0 \\
0.0 \\
0.0 \\
0.0 \\
0.0 \\
0.0 \\
0.0 \\
0.0 \\
0.0 \\
0.0 \\
0.0 \\
0.0 \\
0.0 \\
0.0 \\
0.0 \\
0.0 \\
0.0 \\
0.0 \\
0.0 \\
0.0 \\
0.0 \\
0.0 \\
0.0 \\
0.0 \\
0.0 \\
0.0 \\
0.0 \\
0.0\end{array}$ & $\begin{array}{l}0.0 \\
0.0 \\
0.0 \\
0.0 \\
0.0 \\
0.0 \\
0.0 \\
0.0 \\
0.0 \\
0.0 \\
0.0 \\
0.0 \\
0.0 \\
0.0 \\
0.0 \\
0.0 \\
0.0 \\
0.0 \\
0.0 \\
0.0 \\
0.0 \\
0.0 \\
0.0 \\
0.0 \\
0.0 \\
0.0 \\
0.0 \\
0.0 \\
0.0 \\
0.0 \\
0.0 \\
0.0 \\
0.0 \\
0.0 \\
0.0 \\
0.0 \\
0.0 \\
0.0 \\
0.0 \\
0.0\end{array}$ \\
\hline
\end{tabular}


15AUG 88

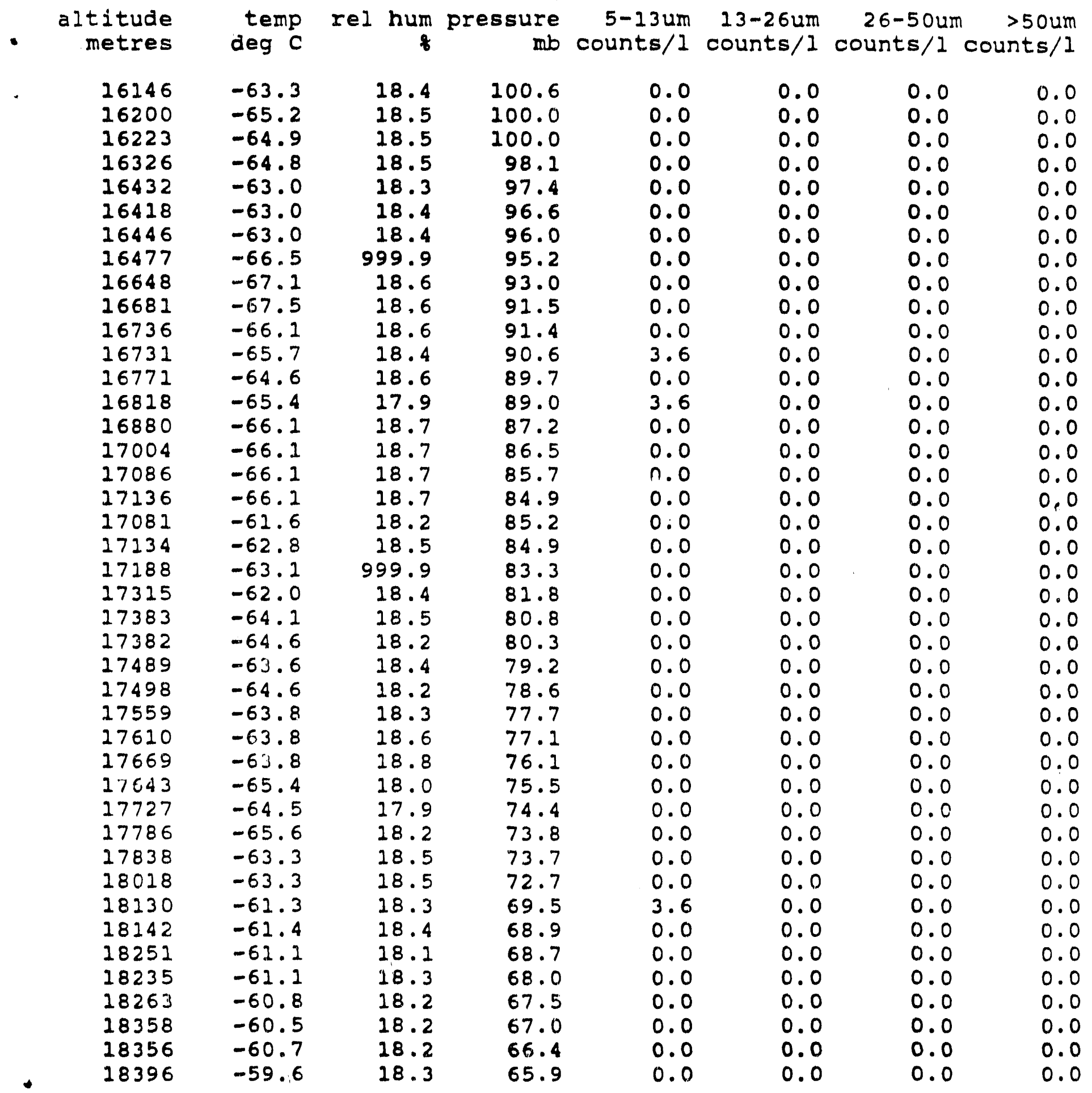


25AUG 88

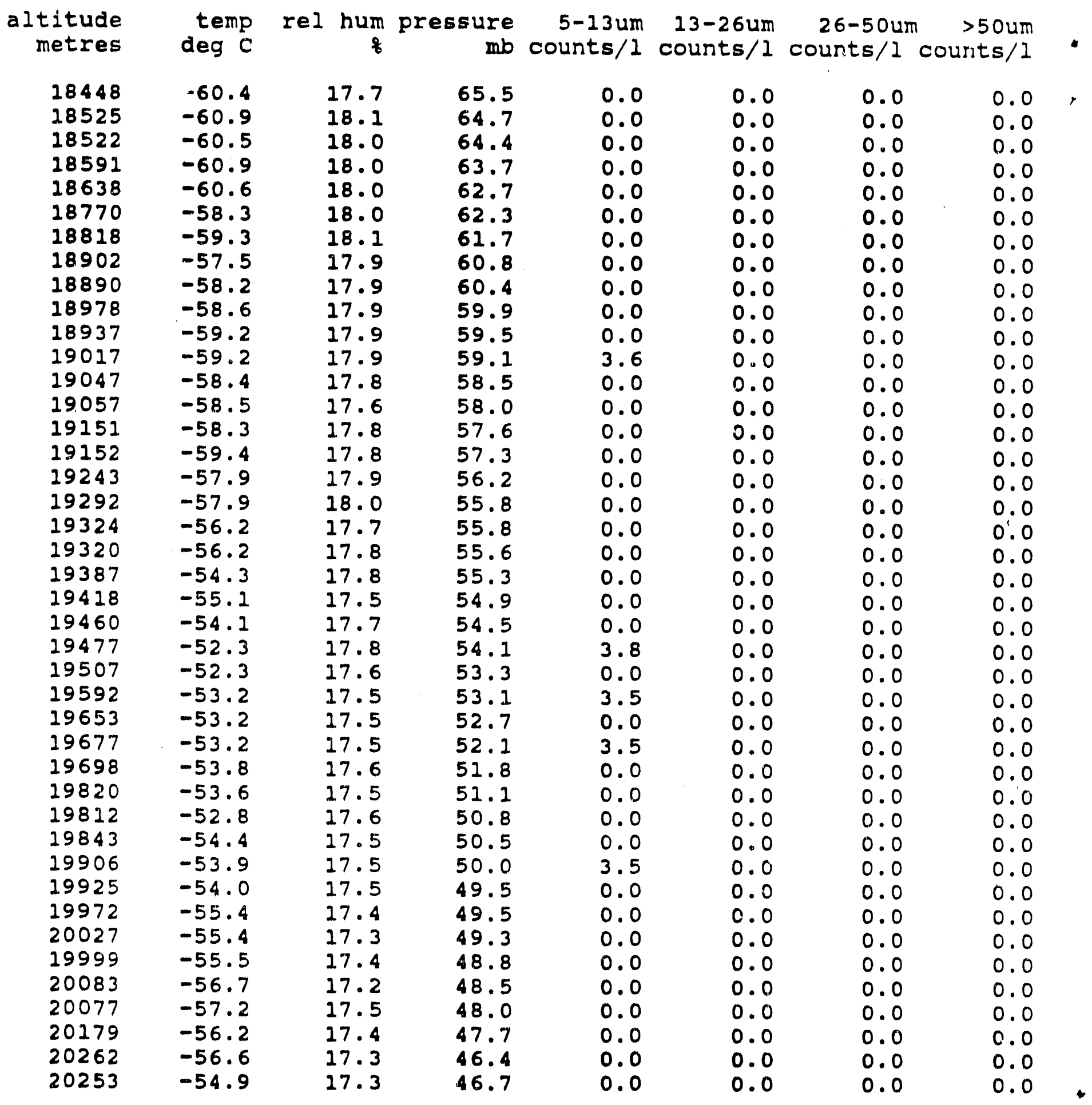


15AUG88

altitude temp rel hum pressure 5-13um 13-26um 26-50um >50um metres

deg $C$

mb counts/1 counts/1 counts/1 counts/1

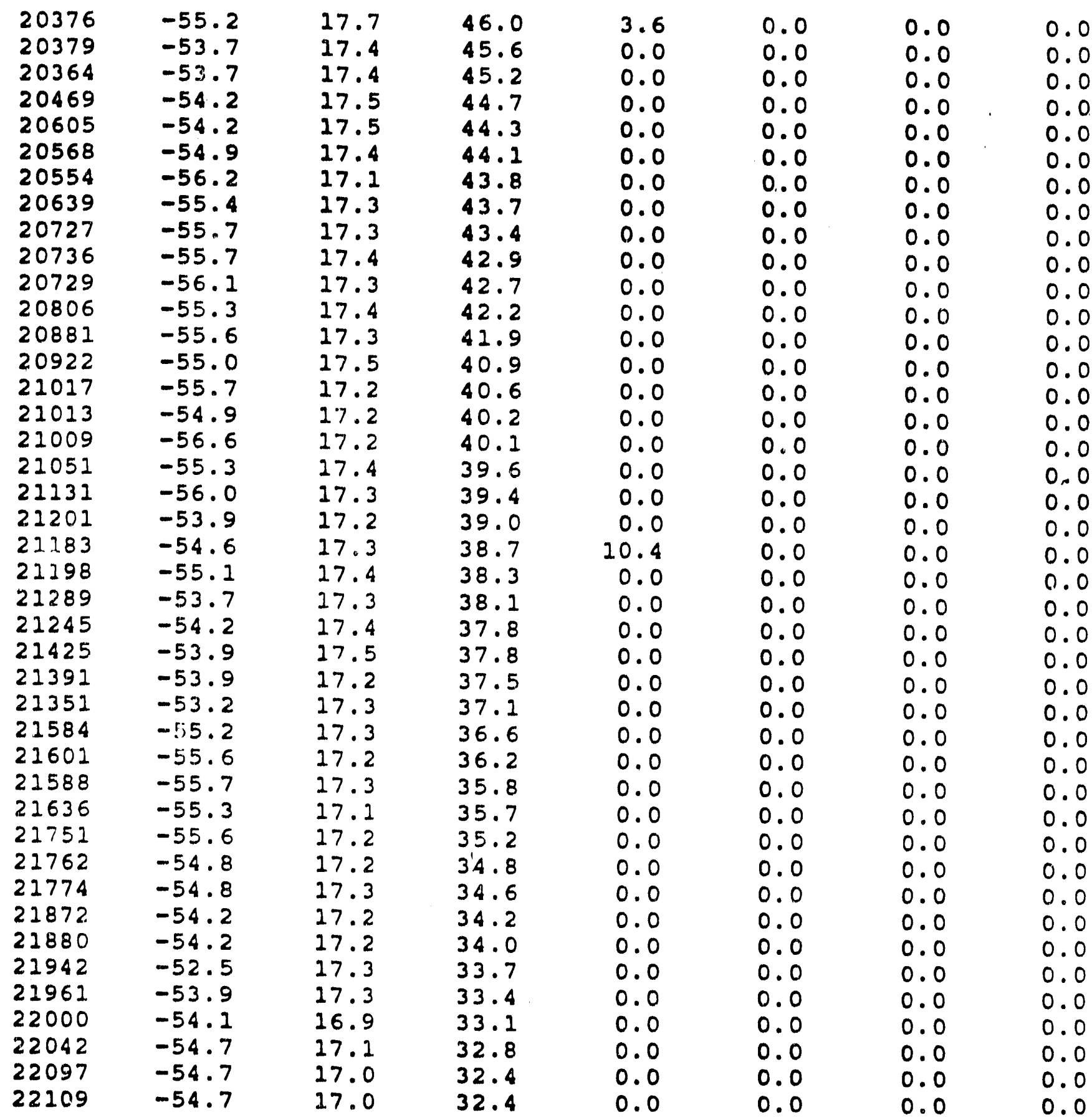




\section{AUG88}

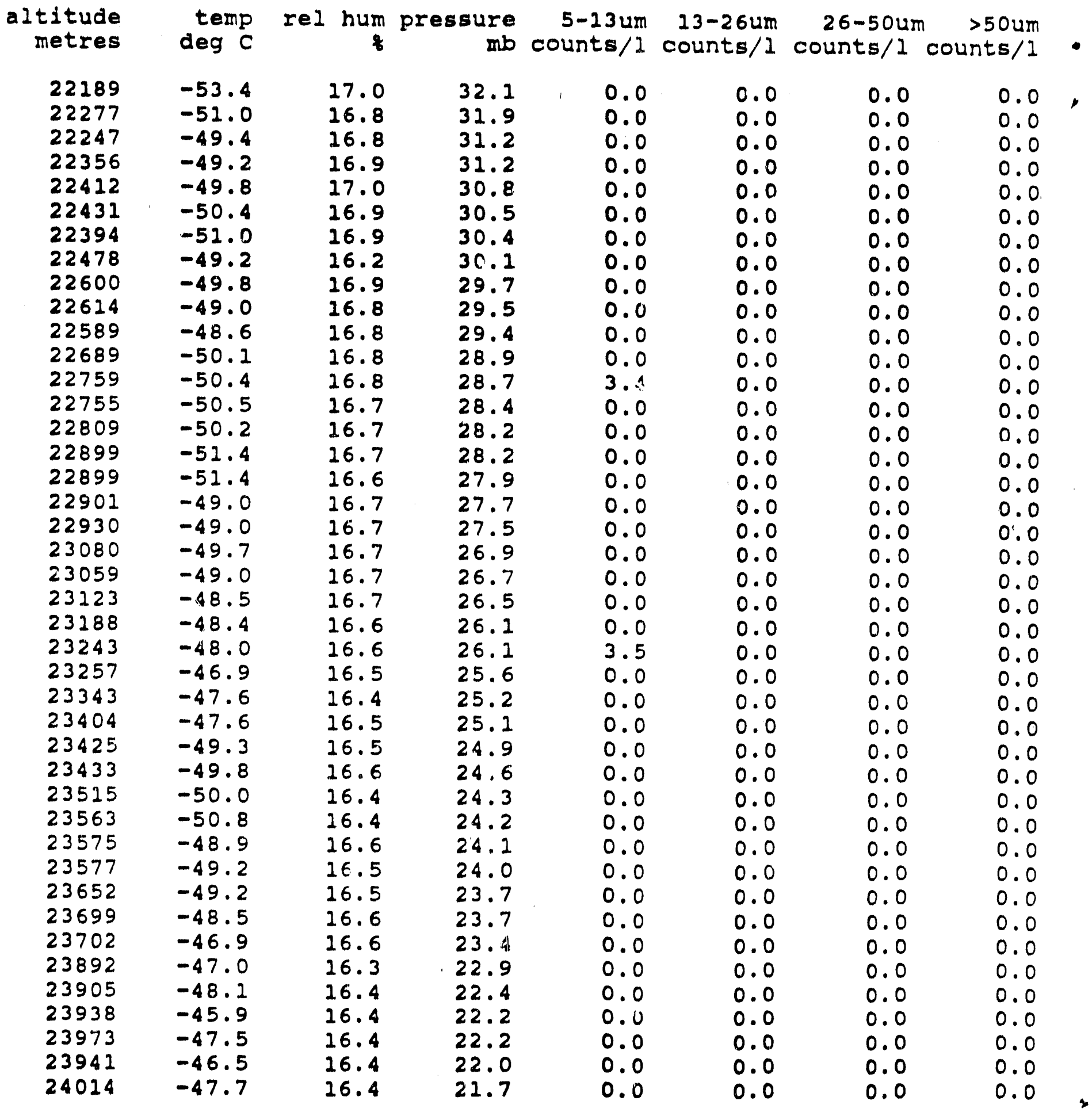


15AUG 88

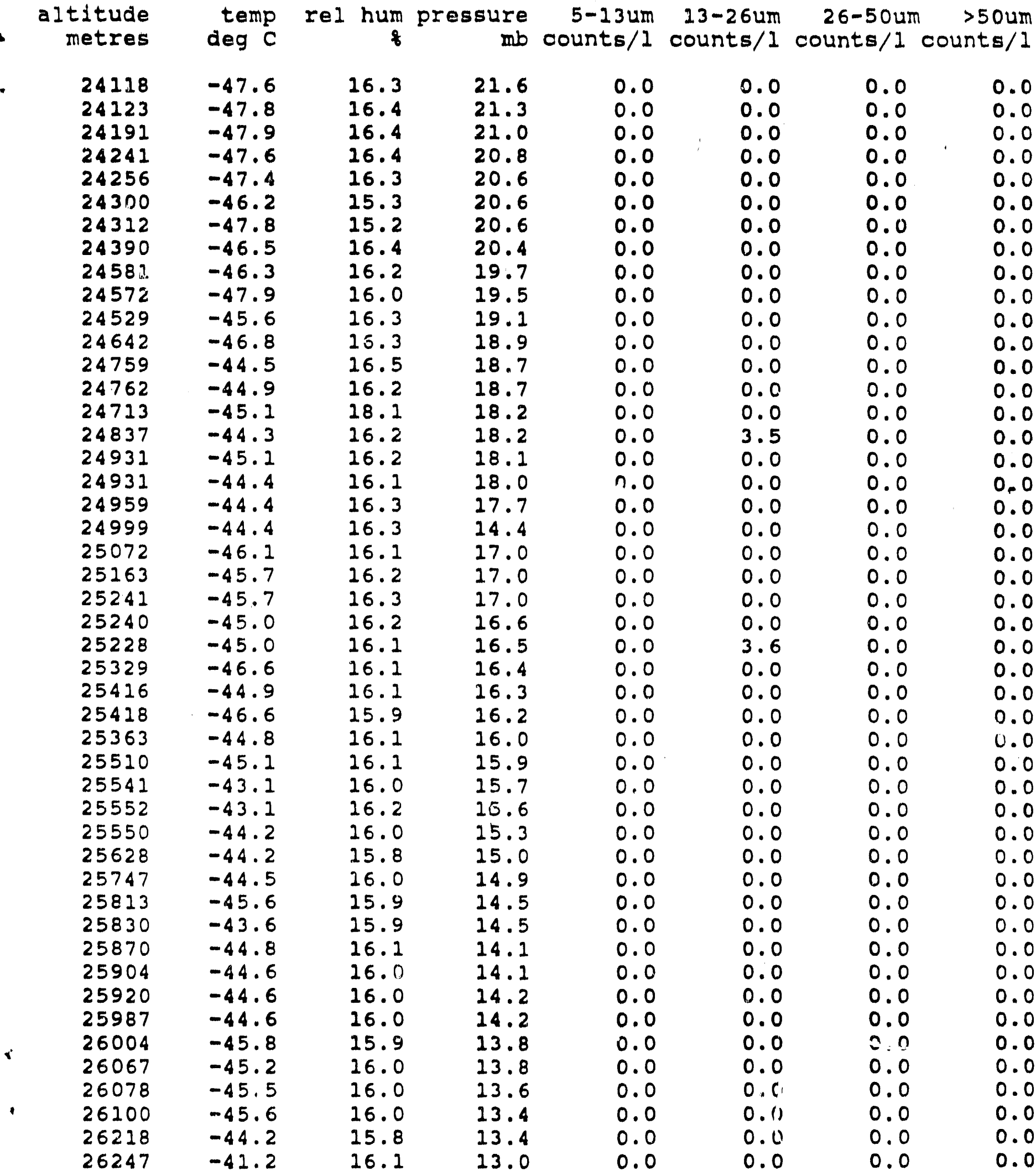




$\begin{array}{llllllll}26274 & -41.2 & 16.0 & 13.0 & 0.0 & 0.0 & 0.0 & 0.0 \\ 26290 & -42.6 & 16.0 & 12.9 & 0.0 & 0.0 & 0.0 & 0.0 \\ 26356 & -42.6 & 16.0 & 12.7 & 0.0 & 0.0 & 0.0 & 0.0 \\ 26372 & -43.3 & 15.8 & 12.6 & 0.0 & 0.0 & 0.0 & 0.0 \\ 26376 & -42.0 & 15.9 & 12.6 & 0.0 & 0.0 & 0.0 & 0.0 \\ 26410 & -42.1 & 15.9 & 12.6 & 0.0 & 0.0 & 0.0 & 0.0 \\ 26777 & -41.8 & 15.8 & 12.6 & 0.0 & 0.0 & 0.0 & 0.0\end{array}$



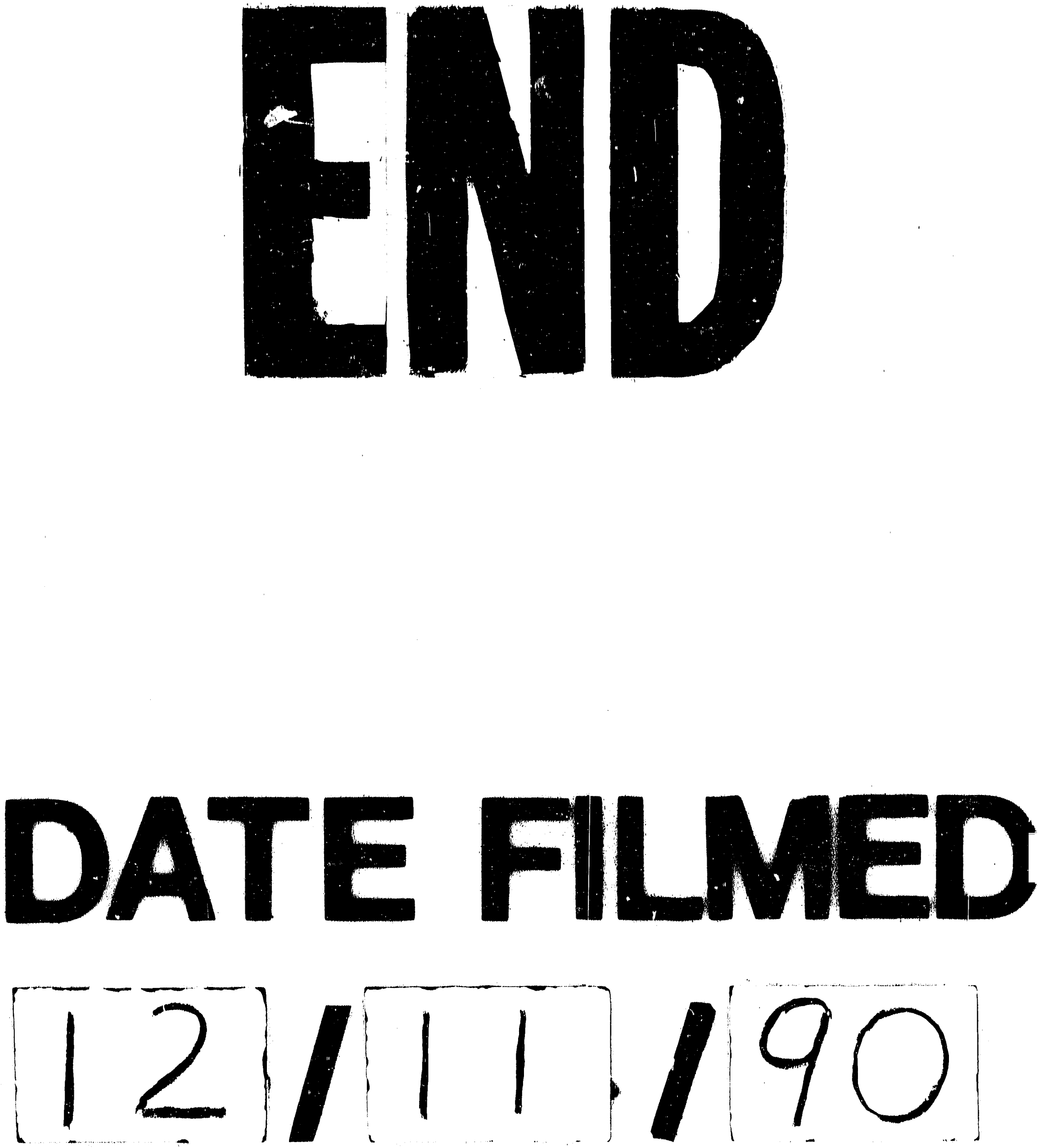